\title{
The Best Inflationary Models After Planck
}

\section{Jérôme Martin, ${ }^{a}$ Christophe Ringeval, ${ }^{b}$ Roberto Trotta $^{c}$ and Vincent Vennin ${ }^{a}$}

${ }^{a}$ Institut d'Astrophysique de Paris, UMR 7095-CNRS, Université Pierre et Marie Curie, 98bis boulevard Arago, 75014 Paris (France)

${ }^{b}$ Centre for Cosmology, Particle Physics and Phenomenology, Institute of Mathematics and Physics, Louvain University, 2 Chemin du Cyclotron, 1348 Louvain-la-Neuve (Belgium)

${ }^{c}$ Imperial College London, Astrophysics \& Imperial Centre for Inference and Cosmology, Blackett Laboratory, Prince Consort Road, London SW7 2AZ (United Kingdom)

E-mail: jmartin@iap.fr, christophe.ringeval@uclouvain.be,r.trotta@imperial.ac.uk, vennin@iap.fr

\begin{abstract}
We compute the Bayesian evidence and complexity of 193 slow-roll single-field models of inflation using the Planck 2013 Cosmic Microwave Background data, with the aim of establishing which models are favoured from a Bayesian perspective. Our calculations employ a new numerical pipeline interfacing an inflationary effective likelihood with the slowroll library ASPIC and the nested sampling algorithm MultiNest. The models considered represent a complete and systematic scan of the entire landscape of inflationary scenarios proposed so far. Our analysis singles out the most probable models (from an Occam's razor point of view) that are compatible with Planck data, while ruling out with very strong evidence $34 \%$ of the models considered. We identify $26 \%$ of the models that are favoured by the Bayesian evidence, corresponding to 15 different potential shapes. If the Bayesian complexity is included in the analysis, only $9 \%$ of the models are preferred, corresponding to only 9 different potential shapes. These shapes are all of the plateau type.
\end{abstract}

Keywords: Cosmic Inflation, Slow-roll, Reheating, Cosmic Microwave Background, Aspic, Bayesian model comparison 


\section{Contents}

1 Introduction $\quad 1$

2 Bayesian inference and model comparison 3

2.1 Bayes factor and posterior model probability 3

2.2 Prior sensitivity considerations $\quad 5$

$\begin{array}{lll}2.3 & \text { Bayesian complexity } & 6\end{array}$

3 Fast Bayesian evidence calculation $\quad 6$

3.1 Effective likelihood via slow-roll reparameterisation 6

3.2 Effective likelihood from Planck $2013 \quad 9$

3.3 Computing the evidences 11

3.4 Fine-tuning issues 12

4 Results and discussion $\quad 14$

5 Conclusions $\quad 16$

$\begin{array}{lr}\text { A Choice of priors for inflationary models } & 19\end{array}$

A.1 Higgs Inflation (HI) 21

A.2 Radiatively Corrected Higgs Inflation (RCHI) 21

A.3 Large Field Inflation (LFI) 22

A.4 Mixed Large Field Inflation (MLFI) 22

A.5 Radiatively Corrected Massive Inflation (RCMI) 22

A.6 Radiatively Corrected Quartic Inflation (RCQI) 23

A.7 Natural Inflation (NI) 23

A.8 Exponential SUSY Inflation (ESI) 24

A.9 Power Law Inflation (PLI) 24

A.10 Kähler Moduli Inflation (KMII) 25

A.11 Horizon Flow Inflation at first order (HF1I) 25

A.12 Coleman Weinberg Inflation (CWI) 25

A.13 Loop Inflation (LI) 26

A.14 $R+R^{2 p}$ Inflation (RpI) $\quad 26$

A.15 Double Well Inflation (DWI) 27

A.16 Mutated Hilltop Inflation (MHI) 27

A.17 Radion Gauge Inflation (RGI) 28

A.18 MSSM Inflation (MSSMI) 28

A.19 Renormalisable Inflection Point Inflation (RIPI) 29

A.20 Arctan Inflation (AI) 29

A.21 Constant ns A Inflation (CNAI) 29

A.22 Constant ns B Inflation (CNBI) 30

A.23 Open String Tachyonic Inflation (OSTI) 30

A.24 Witten-O'Raifeartaigh Inflation (WRI) 31

A.25 Small Field Inflation (SFI) 31

A.26 Intermediate Inflation (II) 32 
A.27 Kähler Moduli Inflation II (KMIII) 33

A.28 Logamediate inflation (LMI) 34

A.29 Twisted inflation (TWI) 35

A.30 Generalised MSSM Inflation (GMSSMI) 36

A.31 Generalised Renormalisable Point Inflation (GRIPI) 40

A.32 Brane SUSY Breaking Inflation (BSUSYBI) 43

A.33 Tip Inflation (TI) 43

A.34 Beta Exponential Inflation (BEI) 45

A.35 Pseudo Natural Inflation (PSNI) 45

A.36 Non Canonical Kähker Inflation (NCKI) 48

$\begin{array}{ll}\text { A.37 Constant Spectrum Inflation (CSI) } & 49\end{array}$

A.38 Orientifold Inflation (OI) 49

A.39 Constant $n_{\mathrm{S}}$ C Inflation (CNCI) 49

A.40 Supergravity Brane Inflation (SBI) 50

A.41 Spontaneous Symmetry Breaking Inflation (SSBI) 50

A.42 Inverse Monomial Inflation (IMI) 51

A.43 Brane Inflation (BI) $\quad 52$

A.44 Running-mass Inflation (RMI) 53

A.45 Valley Hybrid Inflation (VHI) 55

A.46 Dynamical Supersymmetric Inflation (DSI) 56

A.47 Generalised Mixed Large Field Inflation (GMLFI) $\quad 57$

A.48 Logarithmic Potential Inflation (LPI) 58

A.49 Constant $n_{\mathrm{S}}$ D Inflation (CNDI) $\quad 59$

\section{Introduction}

The recent release of the Planck satellite data has had important and profound consequences for our understanding of primordial cosmology. These data clearly support the idea that inflation is the correct description of the physical conditions that prevailed in the early universe since they are in agreement with several important and generic predictions made by the inflationary theory. For instance, a basic property of inflation is that spatial curvature should vanish. And one indeed finds that $100 \Omega_{K}=-0.05_{-0.66}^{+0.65}$ by combining Planck with Wilkinson Microwave Anisotropy Probe (WMAP) large-scale polarisation (denoted WP in Ref. [1]) and Baryon Acoustic Oscillations (BAO) measurements. Another important consequence of the Planck data is the detection of a spectral tilt, $n_{\mathrm{S}}=0.9603 \pm 0.0073$ thus ruling out scale invariance at more than $5 \sigma$, a level of significance predicted in Ref. [2], and convincingly confirming a crucial inflationary prediction. Moreover, the Planck data seem to point to the simplest (but non-trivial) version of inflation. Indeed, neither a significant running nor a significant running of the running have been detected since it is found that $\mathrm{d} n_{\mathrm{S}} / \mathrm{d} \ln k=-0.0134 \pm 0.009$ (Planck+WP) and $\mathrm{d}^{2} n_{\mathrm{S}} / \mathrm{d} \ln ^{2} k=0.02 \pm 0.016$ (Planck+WP), with a pivot scale chosen at $k_{*}=0.05 \mathrm{Mpc}^{-1}$. The data are also compatible with adiabaticity at $95 \% \mathrm{CL}$. If one defines $\alpha_{a b}^{\left(\ell_{\min }, \ell_{\max }\right)} \equiv(\Delta T)_{a b}^{2}\left(\ell_{\min }, \ell_{\max }\right) /(\Delta T)_{\text {tot }}^{2}\left(\ell_{\min }, \ell_{\max }\right)$, with $a, b=\mathcal{R}, \mathcal{I}$, where $\mathcal{I}$ stands for Cold Dark Isocurvature (CDI), Neutrino Density Isocurvature (NDI) or Neutrino Velocity Isocurvature (NVI) and $(\Delta T)_{X}^{2}\left(\ell_{\min }, \ell_{\max }\right)=\sum_{\ell=\ell_{\min }}^{\ell=\ell_{\max }}(2 \ell+1) C_{\ell, X}^{T T}$, then one obtains $\alpha_{\mathcal{R} \mathcal{R}}^{(2,2500)} \in[0,98,1.07]$ and $\alpha_{\mathcal{R} \mathcal{I}}^{(2,2500)} \in[-0.093,0.014]$ for $\mathcal{I}=\mathrm{CDI}, \alpha_{\mathcal{R} \mathcal{R}}^{(2,2500)} \in[0,99,1.09]$ and $\alpha_{\mathcal{R} \mathcal{I}}^{(2,2500)} \in[-0.18,0.0]$ for $\mathcal{I}=$ NDI, $\alpha_{\mathcal{R} \mathcal{R}}^{(2,2500)} \in[0,96,1.05]$ and $\alpha_{\mathcal{R} \mathcal{I}}^{(2,2500)} \in[-0.09,0.026]$ 
for $\mathcal{I}=$ NVI. This implies that isocurvature modes are compatible with zero although the analysis is done with one isocurvature mode at a time only. A quite large non-adiabatic contribution remains possible but, as discussed in Ref. [3], this is in fact driven by the data in the range $\ell \leq 40$. The Planck data also imply that primordial non-Gaussianity is compatible with zero, namely $f_{\mathrm{NL}}^{\text {loc }}=2.7 \pm 5.8, f_{\mathrm{NL}}^{\text {eq }}=-42 \pm 75$ and $f_{\mathrm{NL}}^{\text {ortho }}=-25 \pm 39$ [4]. Some anomalies or "glitches" have also been reported but the corresponding statistical significance is unclear and, in any case, not yet sufficient to claim a detection.

Therefore, the overall picture that emerges is that the inflationary mechanism is nontrivial but, at the same time, "non-exotic". In particular, the complicated scenarios that were considered, at some point, as attractive are now disfavoured (but not necessarily ruled out). Therefore, in accordance with an Occam's razor principle, that the simplest viable explanation for the observations at hand ought to be preferred, it is appropriate to consider - at least for the moment - the simplest scenarios, namely single field slow-roll inflation with a standard kinetic term. This type of scenarios is characterised by one free function, the potential $V(\phi)$. Therefore, identifying the "best model of inflation" boils down to determining the potential $V(\phi)$ which fits the data the best with the smallest number of free parameters and the least fine-tuning.

In order to achieve this task, it is first necessary to identify all the scenarios belonging to the above-mentioned class. This is not so easy since, even if restricted to a small part of the inflationary landscape, the "single-field region" remains densely populated. This was accomplished recently in the "Encyclopadia Inflationaris" of Ref. [5]. Once all the singlefield models have been identified, one needs to quantify statistically whether a model is "better" than another. This question can be addressed in the framework of Bayesian model comparison, which requires the computation of the Bayesian evidence, or global likelihood, i.e. the integral of the likelihood over the prior space for each model. The ratio of such evidences then gives the Bayes factor, representing the degree by which the Planck data have modified our a priori relative belief in each pair of models. From the Bayes factors, one can then evaluate the posterior probability for each model, and thus identify the "best" (in a Bayesian sense) model of inflation. The calculation of the Bayesian evidence of each of the Encyclopadia Inflationaris scenarios constitutes the main subject of the present paper.

This article is organised as follows. In the next section, section 2, we briefly present the theory of Bayesian inference and how it can be used to perform model comparison. In sub-section 2.1, we recall the definition of the Bayesian evidence and, in sub-section 2.2, we discuss how this quantity depends on the prior choices. In sub-section 2.3, we also introduce the Bayesian complexity and explains its meaning. In section 3, we discuss how the Bayesian evidences and complexities can be calculated efficiently and rapidly from the ASPIC ${ }^{1}$ library. In sub-section 3.1, we present the idea behind the method introduced in Ref. [6] (and used in the present article) and, in sub-section 3.2, we detail how the effective likelihood, which is the crucial tool of the method of Ref. [6], can be determined from the Planck 2013 data. In subsection 3.3, we describe the numerical methods used in order to calculate the evidences from the effective likelihood. We also specify the priors chosen on the non-primordial parameters. In sub-section 3.4, we briefly discuss the accuracy of our calculations and its limitations. Then, in section 4 , we present our results, namely the numerical values of the evidence and complexity for all the models considered and we discuss the physical implications of our calculations. In section 5, we summarise our findings and present our conclusions. Finally,

${ }^{1}$ http://cp3.irmp.ucl.ac.be/ ringeval/aspic.html 
in appendix A, we review in detail how the priors, for each model, have been chosen. Special attention has been paid to their physical origin and we discuss how the Bayesian evidence would be modified if the priors were changed.

\section{Bayesian inference and model comparison}

In this section, we briefly review Bayesian inference theory and Bayesian model comparison, which we adopt to compare the performance of the Encyclopadia Inflationaris scenarios.

\subsection{Bayes factor and posterior model probability}

Let $\mathcal{M}_{i}$ be a collection of $N^{\text {mod }}$ models $\left(i=1, \cdots, N^{\text {mod }}\right)$ describing a given physical situation. In this paper, we will denote by "model" a choice of inflationary potential, together with the specification of a prior distribution for its parameters. A given shape of the potential can support different prior choices, and we call the selection of a potential shape (without specification of a prior for its parameters) a "scenario". Thus within a given inflationary scenario there can be multiple models. The following considerations are however fully general. A model $\mathcal{M}_{i}$ is specified by a set of $N_{i}$ parameters $\theta_{i j}$ (with $j=1, \cdots, N_{i}$ ) and by the prior probability distribution of each of its parameters, namely $\pi\left(\theta_{i j} \mid \mathcal{M}_{i}\right)$. In the context of inference on the model's parameter (where the model is assumed to be correct), the prior can be set from the posterior of a previous observation. However, if one is interested in assessing a model's performance via Bayesian model comparison, it is preferable to understand the priors in terms of the a priori available parameter space under the theory represented by model $\mathcal{M}_{i}$ (see e.g. Refs. [7-15] for further details).

Bayesian inference uses Bayes' theorem to update our degree of belief in hypotheses when some new data $D$ becomes available (here, we think of $D$ as the Cosmic Microwave Background - CMB - Planck data but the formalism is generic). Assuming that model $\mathcal{M}_{i}$ is true, from Bayes' theorem, the posterior probability of its parameters $\theta_{i j}$ 's can be expressed as

$$
p\left(\theta_{i j} \mid D, \mathcal{M}_{i}\right)=\frac{1}{\mathcal{E}\left(D \mid \mathcal{M}_{i}\right)} \mathcal{L}\left(\theta_{i j}\right) \pi\left(\theta_{i j} \mid \mathcal{M}_{i}\right),
$$

where $\mathcal{L}\left(\theta_{i j}\right)=p\left(D \mid \theta_{i j}, \mathcal{M}_{i}\right)$ is the likelihood function for the parameters of model $\mathcal{M}_{i}$. The quantity $\mathcal{E}\left(D \mid \mathcal{M}_{i}\right)$ is just a normalisation factor, called the Bayesian evidence or model likelihood, and it is given by

$$
\mathcal{E}\left(D \mid \mathcal{M}_{i}\right)=\int \mathrm{d} \theta_{i j} \mathcal{L}\left(\theta_{i j}\right) \pi\left(\theta_{i j} \mid \mathcal{M}_{i}\right)
$$

If we are only interested in constraining the parameters $\theta_{i j}$ of the model, then the Bayesian evidence can be neglected. However, in the following we shall focus on the question of assessing the posterior model's probability, for which the Bayesian evidence plays a central role.

Using again Bayes' theorem, one obtains the posterior probability of the model $\mathcal{M}_{i}$, which is given by

$$
p\left(\mathcal{M}_{i} \mid D\right)=\frac{\mathcal{E}\left(D \mid \mathcal{M}_{i}\right) \pi\left(\mathcal{M}_{i}\right)}{p(D)}
$$




\begin{tabular}{lll}
\hline$\left|\ln B_{\mathrm{REF}}^{i}\right|$ & Odds & Strength of evidence \\
\hline$<1.0$ & $\lesssim 3: 1$ & Inconclusive \\
1.0 & $\sim 3: 1$ & Weak evidence \\
2.5 & $\sim 12: 1$ & Moderate evidence \\
5.0 & $\sim 150: 1$ & Strong evidence \\
\hline
\end{tabular}

Table 1. Jeffreys' scale for evaluating the strength of evidence when comparing two models, $\mathcal{M}_{i}$ versus a reference model $\mathcal{M}_{\mathrm{REF}}$, here slightly modified following the prescriptions given in Refs. [15, 16].

where $\pi\left(\mathcal{M}_{i}\right)$ is the prior belief in model $\mathcal{M}_{i}$. The quantity $p(D)$ is a normalisation factor (which only depends on the data but not on the model under consideration), given by

$$
p(D)=\sum_{i} \mathcal{E}\left(D \mid \mathcal{M}_{i}\right) \pi\left(\mathcal{M}_{i}\right)
$$

When comparing two models against each other, this factor cancels. If one defines a "reference model", $\mathcal{M}_{\mathrm{REF}}$, against which all other models are compared, the posterior odds between a model $\mathcal{M}_{i}$ and the reference model are given by

$$
\frac{p\left(\mathcal{M}_{i} \mid D\right)}{p\left(\mathcal{M}_{\mathrm{REF}} \mid D\right)}=B_{\mathrm{REF}}^{i} \frac{\pi\left(\mathcal{M}_{i}\right)}{\pi\left(\mathcal{M}_{\mathrm{REF}}\right)} .
$$

Here, we have introduced the Bayes factor $B_{\mathrm{REF}}^{i}$ which can be expressed as the ratio of the evidences, namely

$$
B_{\mathrm{REF}}^{i} \equiv \frac{\mathcal{E}\left(D \mid \mathcal{M}_{i}\right)}{\mathcal{E}\left(D \mid \mathcal{M}_{\mathrm{REF}}\right)}
$$

Under the principle of indifference, we can assume non-committal model priors, i.e. we give all models the same a priori probability, $\pi\left(\mathcal{M}_{i}\right)=1 / N^{\text {mod }}$, in which case the Bayes factor becomes identical with the posterior odds. With this assumption, a Bayes factor larger (smaller) than one means a preference for the model $\mathcal{M}_{i}$ over the reference model (a preference for the reference model over $\mathcal{M}_{i}$ ). The "Jeffreys' scale", see Table 1, gives an empirical prescription for translating the values of $B_{\mathrm{REF}}^{i}$ into strengths of belief.

With non-committal model priors, the posterior probability for model $\mathcal{M}_{i}$ is then given by

$$
p\left(\mathcal{M}_{i} \mid D\right)=\frac{B_{\mathrm{REF}}^{i}}{\sum_{j} B_{\mathrm{REF}}^{j}} .
$$

This implicitly further assumes that the list of $N^{\text {mod }}$ is reasonably complete - i.e. that there isn't a yet undiscovered better models that have not been considered a priori (see Ref. [17] for a Bayesian method leading to the discovery of such unknown models).

The fundamental idea underpinning Bayesian model comparison is that "economic" models that fit well the data while exhibiting strong predictivity are rewarded, while models with a large number of free parameters that turn out not to be required by the data are penalised for the wasted parameter space. Therefore, in a Bayesian sense, the "best" model is the one that achieves the best compromise between quality of fit and simplicity (see Ref. $[15,18]$ for further details and Ref. $[19,20]$ for a discussion of issues in Bayesianfrequentist calibrations). One of the attractive features of Bayesian model comparison is 
that it automatically embodies a quantitative version of Occam's razor, that is to say, the principle of simplicity (see Ref. [21] for a critical discussion and comparison with frequentist methods). The price to pay is that the Occam's razor effect depends in an irreducible way on the choice of prior (and particularly on its range) hence the latter must be set according to physical considerations stemming from the model. We now turn to the crucial question of prior sensitivity.

\subsection{Prior sensitivity considerations}

As mentioned above, since the priors $\pi\left(\theta_{i j} \mid \mathcal{M}_{i}\right)$ play a crucial role, a detailed description on how they have been chosen is provided for each model in appendix A. We also discuss how the evidence is affected by alternative prior choices within various theoretical scenarios. For this reason, the number of evidences presented in this paper is much larger than the number Encyclopadia Inflationaris scenarios. Indeed, a given field potential can support several prior choices motivated by different theories, each of them leading to different evidences. We thus consider them as different models.

For each field potential, physical considerations have been used to determine the shape of the prior. If a parameter is small but its order of magnitude is unknown, as it is typically the case for a coupling constant used in a perturbative expansion, then a Jeffreys' prior (uniform in the logarithm of the parameter) is the most uninformative. If, on the contrary, we deal with a parameter whose order of magnitude is known, then this is a scale parameter and a uniform prior on the parameter itself is appropriate. As priors must be proper (i.e., normalised), the support of the prior $\left[\theta_{\min }, \theta_{\max }\right]$ must also be chosen according to the natural values allowed by the underlying physical scenario. Indeed, the strength of the Occam's razor effect depends on this range, as generically the Bayesian evidence scales as (for uniform priors)

$$
\mathcal{E}\left(D \mid \mathcal{M}_{i}\right) \propto \frac{1}{\theta_{\max }-\theta_{\min }},
$$

for cases where the support of the likelihood is much smaller than the support of the prior. However, since the Jeffreys' scale is logarithmic in the Bayes factor, the dependence on the prior range is relatively mild. Still, there are many cases in which $\theta_{\min }$ and $\theta_{\max }$ remain unspecified by the model. When this happens, attention has been paid on how the evidence is affected when this range is modified.

From the above argument it follows that one can estimate the variation in the evidence that one would get from a change of the range of the prior simply by rescaling it proportionally to the ratio of the prior volumes in the parameter space. This holds approximately true as long as the support of the likelihood is well within that of the prior. This is more detailed in appendix A where, if necessary, we discuss for each model how this calculation can be done in practice.

Another often-encountered situation is when the likelihood is flat along the $\theta_{i k}$ direction, i.e. the data are insensitive to one of the parameters of the model under consideration. In this case, the posterior for that parameter is identical to the prior and the Bayes factor reduces to unity - the Bayesian evidence is insensitive to the number of unconstrained parameters in a model. For such flat directions in parameter space, the prior boundary does not matter (as long as the likelihood stays flat), and the evidence is unchanged by a rescaling of the boundaries of the prior. A second quantity is thus required to measure the number of effective parameters that the data can constrain in a given model. This can be implemented in various way, as for instance by using Kullback-Leiber divergence between the prior and the posterior, leading to the notion of model complexity that we now discuss $[15,22,23]$. 


\subsection{Bayesian complexity}

The number of parameters in a model is a poor description of its "complexity", as parameters that are not constrained by the data should not be counted. A better evaluation of complexity (in a Bayesian sense) has been introduced by [24], who advocates using the relative entropy between the prior and the posterior distribution (i.e., the Kullback-Leibler divergence) as a better suited measure of the number of free parameters in a model that the data can actually constrain.

As shown in Ref. [23], such an effective number of parameters, or Bayesian complexity, $\mathcal{C}$, can be written as

$$
\mathcal{C}_{i}=\left\langle-2 \log \mathcal{L}\left(\theta_{i j}\right)\right\rangle+2 \log \mathcal{L}\left(\theta_{i j}^{\mathrm{ML}}\right),
$$

where $\langle\cdot\rangle$ denotes averaging over the posterior $p\left(\theta_{i j} \mid D, \mathcal{M}_{i}\right)$ and $\theta_{i j}^{\mathrm{ML}}$ is the maximumlikelihood estimate of the model's parameters which can be approximately obtained from the posterior samples used to map out the posterior distribution ${ }^{2}$. The Bayesian complexity is thus not an absolute measure of the number of constrained parameters - rather it assesses the constraining power of the data with respect to the measure provided by the prior.

The use of model complexity together with the Bayesian evidence allows us to distinguish between cases where $\mathcal{E}\left(D \mid \mathcal{M}_{i}\right) \simeq \mathcal{E}\left(D \mid \mathcal{M}_{j}\right)$ (i.e., two models exhibiting approximately the same Bayesian evidence) but $\mathcal{C}_{i} \simeq \mathcal{C}_{j}$, in which case the data is insufficient to distinguish between the two models (as their effective complexities are the same); or the case where $\mathcal{C}_{i}>\mathcal{C}_{j}$, which means that the data are sufficient to measure extra parameters of model $i$ but that those parameters are not required by the evidence, in which case we ought to prefer model $j$, as the one with the smallest (measured) complexity.

\section{Fast Bayesian evidence calculation}

The computation of the Bayesian evidence can be a numerically demanding task, as it requires the evaluation of the multi-dimensional integral of Eq. (2.2). This is particularly computationally intensive for Markov Chains Monte-Carlo (MCMC)-based methods. In recent years, a powerful tool has emerged in the shape of nested sampling, and its implementation in the MultiNest code [26, 27]. Even with such a highly efficient algorithm, the Bayesian evidence requires hundreds of thousands of likelihood evaluations for each model. A typical analysis based on the Planck likelihood coupled with an exact inflationary code to integrate the perturbations requires roughly $3 \times 10^{5} \mathrm{CPU}$ hours (or 3.4 CPU years) of computing time on modern x86_64 processors. Performing this for each model considered here would become prohibitively time consuming, even with high-performance computing.

In this section, we briefly describe the method introduced in Ref. [6] which allows us to calculate the Bayesian evidences in a fraction of the time that would be required using conventional tools. We also mention the limitation of the method, especially the fact that the very low evidences may be poorly approximated.

\subsection{Effective likelihood via slow-roll reparameterisation}

Let us denote by $a_{\ell m}^{\text {obs }}$ the CMB temperature map recently observed by the Planck satellite. From this map, one can estimate the measured multipole moments $C_{\ell}^{\text {obs }}=\left\langle a_{\ell m}^{\text {obs }} a_{\ell m}^{\text {obs } \star}\right\rangle$. From the $\Lambda \mathrm{CDM}$ model (or any other post-inflationary history) and the scenario of inflation, one

\footnotetext{
${ }^{2}$ See however Ref. [25] for the caveats that apply when one wants to derive maximum likelihood estimates from Bayesian posterior maps.
} 
can compute the theoretical prediction for those multipole moments, $C_{\ell}^{\mathrm{th}}\left(\theta_{\mathrm{s}}, \theta_{\mathrm{reh}}, \theta_{\text {inf }}\right)$ as a function of the parameters in the model. Here, $\theta_{\mathrm{s}}$ represents a set of parameters describing post-inflationary physics, see Eq. (3.12) for a precise definition, $\theta_{\text {reh }}$ are the parameters of reheating and $\theta_{\text {inf }}$ describe the shape of the potential $V(\phi)$. The reheating epoch can be described either with $\theta_{\text {reh }}=\left(\rho_{\text {reh }}, \bar{w}_{\text {reh }}\right)$, namely the energy density of the universe at the end of reheating and the mean equation of state parameter during reheating; or with the completely generic rescaled reheating parameter $\theta_{\text {reh }}=\ln (R)$, defined by

$$
R \equiv R_{\mathrm{rad}} \frac{\rho_{\mathrm{end}}^{1 / 4}}{M_{\mathrm{Pl}}}, \quad R_{\mathrm{rad}} \equiv \frac{a_{\mathrm{end}}}{a_{\mathrm{reh}}}\left(\frac{\rho_{\mathrm{end}}}{\rho_{\mathrm{reh}}}\right)^{1 / 4} .
$$

Here the indices "end" and "reh" denote the end of inflation and end of the reheating era (i.e. the beginning of the radiation dominated era, see Ref. [5] for further details), $\rho$ and $a$ being the energy density of the universe and the FLRW scale factor, respectively. Here, we have chosen to sample over the same optimised set discussed in Refs. [6, 28-30], see also Refs. [31, 32]. All possible reheating histories are sampled using the rescaled reheating parameter and with a prior uniform in its logarithm,

$$
\pi\left(\theta_{\text {reh }}\right)=\pi[\ln (R)]=U(-46,15) .
$$

The boundaries of the prior support encompass all reheating histories satisfying the constraints that the mean equation of state during reheating verifies $-1 / 3<\bar{w}_{\text {reh }}<1$, and $\rho_{\text {nuc }}<\rho_{\text {reh }}<\rho_{\text {end }}$. The last inequality enforces that reheating takes place after inflation and before Big-Bang Nucleosynthesis $(\mathrm{BBN})$. Practically, we have chosen $\rho_{\text {nuc }}^{1 / 4} \equiv 10 \mathrm{MeV}$. More details can be found in Refs. [28-30, 33-37].

The expression for $C_{\ell}^{\text {th }}$ can be written as

$$
C_{\ell}^{\text {th }}\left(\theta_{\mathrm{s}}, \theta_{\text {reh }}, \theta_{\text {inf }}\right)=\int_{0}^{+\infty} \frac{\mathrm{d} k}{k} j_{\ell}\left(k r_{\ell \mathrm{ss}}\right) T\left(k ; \theta_{\mathrm{s}}\right) \mathcal{P}_{\zeta}\left(k ; \theta_{\text {reh }}, \theta_{\text {inf }}\right),
$$

$j_{\ell}$ being a spherical Bessel function, $r_{\ell s s}$ the comoving radial distance to the last scattering surface, $T\left(k ; \theta_{\mathrm{S}}\right)$ the transfer function which describes the evolution of cosmological perturbations during the standard Friedmann-Lemaitre eras and $\mathcal{P}_{\zeta}$ the inflationary power spectrum.

The posterior distribution for the parameters of interest is given by

$$
p\left(\theta_{\mathrm{s}}, \theta_{\text {reh }}, \theta_{\text {inf }} \mid a_{\ell m}^{\mathrm{obs}}\right)=\frac{1}{\mathcal{E}} \mathcal{L}\left(\theta_{\mathrm{s}}, \theta_{\text {reh }}, \theta_{\text {inf }}\right) \pi\left(\theta_{\mathrm{s}}, \theta_{\text {reh }}, \theta_{\text {inf }}\right),
$$

where $\mathcal{L}\left(\theta_{\mathrm{s}}, \theta_{\text {reh }}, \theta_{\text {inf }}\right)=p\left(a_{\ell m}^{\text {obs }} \mid \theta_{\mathrm{s}}, \theta_{\text {reh }}, \theta_{\text {inf }}\right) \propto e^{-\chi^{2}\left(\theta_{\mathrm{s}}, \theta_{\text {reh }}, \theta_{\text {inf }}\right) / 2}$ is the likelihood function (and the normalisation constant in front is irrelevant), $\chi^{2}$ being the effective chi-squared. The prior distribution $\pi\left(\theta_{\mathrm{s}}, \theta_{\text {reh }}, \theta_{\text {inf }}\right)$ describes our a priori state of knowledge about the values of the parameters before our information is updated. Notice that, for clarity, we have dropped the dependence on the model $\mathcal{M}$ under scrutiny. In Eq. (3.4), $\mathcal{E}$ is the Bayesian evidence discussed in the previous section and reads

$$
\mathcal{E}=\int \mathrm{d} \theta_{\mathrm{s}} \mathrm{d} \theta_{\text {reh }} \mathrm{d} \theta_{\text {inf }} \mathcal{L}\left(\theta_{\mathrm{s}}, \theta_{\text {reh }}, \theta_{\text {inf }}\right) \pi\left(\theta_{\mathrm{s}}, \theta_{\text {reh }}, \theta_{\text {inf }}\right)
$$

It is the quantity we need to calculate for the 193 models considered here. 
The effective chi-squared, and, therefore, the likelihood function, is a function of $C_{\ell}^{\text {th }}$ and of the data, namely

$$
\chi^{2}\left(\theta_{\mathrm{s}}, \theta_{\mathrm{reh}}, \theta_{\mathrm{inf}}\right)=\chi^{2}\left[C_{\ell}^{\mathrm{th}}\left(\theta_{\mathrm{s}}, \theta_{\mathrm{reh}}, \theta_{\mathrm{inf}}\right), a_{\ell m}^{\mathrm{obs}}, \Sigma\right],
$$

where $\Sigma$ is the noise covariance matrix of the measurement. The above expression is only illustrative - in practice one has to deal with more complex issues, including foregrounds, instrumental systematics and the measurements of polarisation in addition to temperature [38]. Assuming that the post-inflationary physics is the same for all inflationary scenarios, different models have different evidences because they have a different power spectrum $\mathcal{P}_{\zeta}\left(k ; \theta_{\text {reh }}, \theta_{\text {inf }}\right)$. In order to calculate the evidence of a given inflationary model, one must therefore evaluate $\mathcal{P}_{\zeta}\left(k ; \theta_{\text {reh }}, \theta_{\text {inf }}\right)$ for the sampled values of $\theta_{\text {reh }}$ and $\theta_{\text {inf }}$, then perform the integral (3.5). In general, $\mathcal{P}_{\zeta}\left(k ; \theta_{\text {reh }}, \theta_{\text {inf }}\right)$ is only known numerically and this procedure is computationally intensive.

It is, however, possible to speed up dramatically this calculation if one uses the fact that the inflationary models under consideration here are all slow-roll models. In that case, there exists a general parametrisation of the power spectrum which is given by $\left(k_{*}\right.$ is the pivot scale)

$$
\begin{aligned}
\mathcal{P}_{\zeta}(k) & =\mathcal{P}_{0}\left[a_{0}\left(\epsilon_{n}\right)+a_{1}\left(\epsilon_{n}\right) \ln \left(\frac{k}{k_{*}}\right)+\frac{1}{2} a_{2}\left(\epsilon_{n}\right) \ln ^{2}\left(\frac{k}{k_{*}}\right)+\ldots\right] \\
& =P_{*}\left[1+\frac{a_{1}\left(\epsilon_{n}\right)}{a_{0}\left(\epsilon_{n}\right)} \ln \left(\frac{k}{k_{*}}\right)+\frac{a_{2}\left(\epsilon_{n}\right)}{a_{0}\left(\epsilon_{n}\right)} \ln ^{2}\left(\frac{k}{k_{*}}\right)+\ldots\right]
\end{aligned}
$$

where $\epsilon_{n}$ are the Hubble-flow parameters evaluated at Hubble exit and $\mathcal{P}_{0}$ represents the overall normalisation $[39,40]$. We have rendered explicit the well-measured quantity $P_{*}=$ $a_{0}\left(\epsilon_{n}\right) \mathcal{P}_{0}=\mathcal{P}_{\zeta}\left(k_{*}\right)$ which fixes the amplitude of the CMB anisotropies. The explicit form of the $a_{i}$ 's as functions of $\epsilon_{n}$ is known [41].

Furthermore, one can express the Hubble flow parameters as a function of the more fundamental inflationary parameters for every scenario. The explicit functionals $\epsilon_{n}\left(\theta_{\text {reh }}, \theta_{\text {inf }}\right)$ are all provided in the ASPIC library and in the Encyclopadia Inflationaris.

The central idea, introduced in [6], is that the likelihood function entering the evidence is invariant under a reparameterisation of the primordial power spectrum parameters. We can thus rewrite the multipole moments (and hence the likelihood function which depends on them $)$ as $C_{\ell}^{\text {th }}\left(\theta_{\mathrm{s}}, \theta_{\text {reh }}, \theta_{\text {inf }}\right)=C_{\ell}^{\text {th }}\left[\theta_{\mathrm{s}}, P_{*}\left(\theta_{\text {reh }}, \theta_{\text {inf }}\right), \epsilon_{n}\left(\theta_{\text {reh }}, \theta_{\text {inf }}\right)\right]$. The evidence of Eq. $(3.5)$ becomes

$$
\begin{aligned}
\mathcal{E} & =\int \mathrm{d} \theta_{\mathrm{s}} \mathrm{d} \theta_{\text {reh }} \mathrm{d} \theta_{\text {inf }} \mathcal{L}\left[\theta_{\mathrm{s}}, P_{*}\left(\theta_{\text {reh }}, \theta_{\text {inf }}\right), \epsilon_{n}\left(\theta_{\text {reh }}, \theta_{\text {inf }}\right)\right] \pi\left(\theta_{\mathrm{s}}\right) \pi\left(\theta_{\text {reh }}, \theta_{\text {inf }}\right) \\
& =\int \mathrm{d} \theta_{\text {reh }} \mathrm{d} \theta_{\text {inf }} \mathcal{L}_{\text {eff }}\left[P_{*}\left(\theta_{\text {reh }}, \theta_{\text {inf }}\right), \epsilon_{n}\left(\theta_{\text {reh }}, \theta_{\text {inf }}\right)\right] \pi\left(\theta_{\text {reh }}\right) \pi\left(\theta_{\text {inf }}\right),
\end{aligned}
$$

where we have defined the effective likelihood, marginalised over the post-inflationary parameters, $\theta_{\mathrm{s}}$, as

$$
\mathcal{L}_{\text {eff }}\left[P_{*}\left(\theta_{\text {reh }}, \theta_{\text {inf }}\right), \epsilon_{n}\left(\theta_{\text {reh }}, \theta_{\text {inf }}\right)\right] \equiv \int \mathrm{d} \theta_{\mathrm{s}} e^{-\frac{1}{2} \chi^{2}\left[C_{\ell}^{\text {th }}\left(\theta_{\mathrm{s}}, P_{*}, \epsilon_{n}\right), a_{\ell m}^{\text {obs }}, \Sigma\right]} \pi\left(\theta_{\mathrm{s}}\right) .
$$


In Eq. (3.9) we have made the reasonable assumption that the prior on the post-inflationary, reheating and primordial parameters are separable ${ }^{3}$, i.e.

$$
\pi\left(\theta_{\mathrm{s}}, \theta_{\text {reh }}, \theta_{\text {inf }}\right)=\pi\left(\theta_{\mathrm{s}}\right) \pi\left(\theta_{\text {reh }}\right) \pi\left(\theta_{\text {inf }}\right) .
$$

The effective likelihood, Eq. (3.10), can be computed as a function of the slow-roll parameters, $P_{*}, \epsilon_{n}$, using machine-learning algorithms to interpolate the functional form of $\mathcal{L}_{\text {eff }}\left(P_{*}, \epsilon_{n}\right)$. Seen as a function of the slow-roll parameters, $\mathcal{L}_{\text {eff }}$ needs only to be computed once for all inflationary models considered here. To then use it for a specific inflationary model, it is sufficient to map its potential parameters $\theta_{\text {inf }}$ and reheating parameters $\theta_{\text {reh }}$ onto the corresponding functionals, $P_{*}\left(\theta_{\mathrm{inf}}, \theta_{\mathrm{reh}}\right), \epsilon_{n}\left(\theta_{\mathrm{inf}}, \theta_{\mathrm{reh}}\right)$.

The computational advantages of our method are twofold. First, the evaluation of the effective likelihood is very fast, since it is obtained as the output of a neural network interpolator (typically, one evaluation requires less than a $\mu$ s of CPU-time on standard x86_64 processor). Second, by integrating out once and for all the post-inflationary parameters from the likelihood, we are left with a much reduced parameter space over which the Bayesian evidence integral has to be computed. The dimensionality of $\theta_{\text {inf }}$ is at most three, while the reheating is described by just one parameter, so that the Bayesian evidence integral is at most four-dimensional. Thanks to this vastly increased efficiency, we were able to compute a large number of Bayesian evidences with a much reduced numerical effort. More details about the method can be found in Ref. [6].

\subsection{Effective likelihood from Planck 2013}

In order to determine $\mathcal{L}_{\text {eff, }}$, we have used the Planck 2013 data [42] together with the second order slow-roll expansion of the primordial power spectra for both the scalar and tensor perturbations. The full Planck likelihood is provided by the Planck collaboration [38]. Concerning the post-inflationary universe, it is assumed to be a flat $\Lambda \mathrm{CDM}$ model such that the parameters $\theta_{\mathrm{s}}$ are:

$$
\begin{aligned}
& \theta_{\mathrm{s}}=\left(\Omega_{\mathrm{b}} h^{2}, \Omega_{\mathrm{dm}} h^{2}, \tau, 100 \theta_{\mathrm{MC}}, A_{100}^{\mathrm{PS}}, A_{143}^{\mathrm{PS}}, A_{217}^{\mathrm{PS}}, r_{143 \times 217}^{\mathrm{PS}}, A_{143}^{\mathrm{CIB}}, A_{217}^{\mathrm{CIB}}, r_{143 \times 217}^{\mathrm{CIB}}, \gamma^{\mathrm{CIB}},\right. \\
& \left.\quad A_{\mathrm{tsZ}}, A_{\mathrm{kSZ}}, \xi^{\mathrm{tSZ} \times \mathrm{CIB}}, c_{100}, c_{217}, \beta_{1}^{1}\right) .
\end{aligned}
$$

The usual $\Lambda$ CDM parameters are the density of baryons $\Omega_{\mathrm{b}}$, of cold dark matter $\Omega_{\mathrm{dm}}$, the reduced Hubble parameter today $h$, the Thompson optical depth $\tau$ to last scattering and an angle, $\theta_{\mathrm{MC}}$, related to the angular size of the sound horizon on the last scattering surface [43]. The remaining parameters describe astrophysical signals on top of the CMB and any relevant instrumental distortions, as they have been modelled by the Planck collaboration [1]. They are the power contribution at $\ell=3000$ of unresolved point sources at $100 \mathrm{GHz}$, at $143 \mathrm{GHz}$, at $217 \mathrm{GHz}$ and their cross correlation $\left(A_{100}^{\mathrm{PS}}, A_{143}^{\mathrm{PS}}, A_{217}^{\mathrm{PS}}, r_{143 \times 217}^{\mathrm{PS}}\right)$. The next are their equivalent for the Cosmic Infrared Background (CIB), namely $A_{143}^{\mathrm{CIB}}, A_{217}^{\mathrm{CIB}}, r_{143 \times 217}^{\mathrm{CIB}}$, and $\gamma^{\mathrm{CIB}}$ stands for the spectral index of the CIB angular power spectrum. The Sunyaev-Zel'dovich (SZ) signals, either thermal or kinetic, and their correlations with the CIB are encoded in the parameters

$A_{\mathrm{tsz}}, A_{\mathrm{kSZ}}, \xi^{\mathrm{tSZ} \times \mathrm{CIB}}$. Finally, calibration and beam uncertainties are taken into account in the last three parameters. More details on how these signals are accounted for can be found in Ref. [38].

\footnotetext{
${ }^{3}$ More precisely, it is sufficient to require that $\pi\left(\theta_{\mathrm{s}}, \theta_{\text {reh }}, \theta_{\text {inf }}\right)=\pi\left(\theta_{\mathrm{s}}\right) \pi\left(\theta_{\text {reh }}, \theta_{\text {inf }}\right)$. However, it is sensible to assume that the reheating and inflationary parameters are separable, too, thus leading to Eq. (3.11).
} 

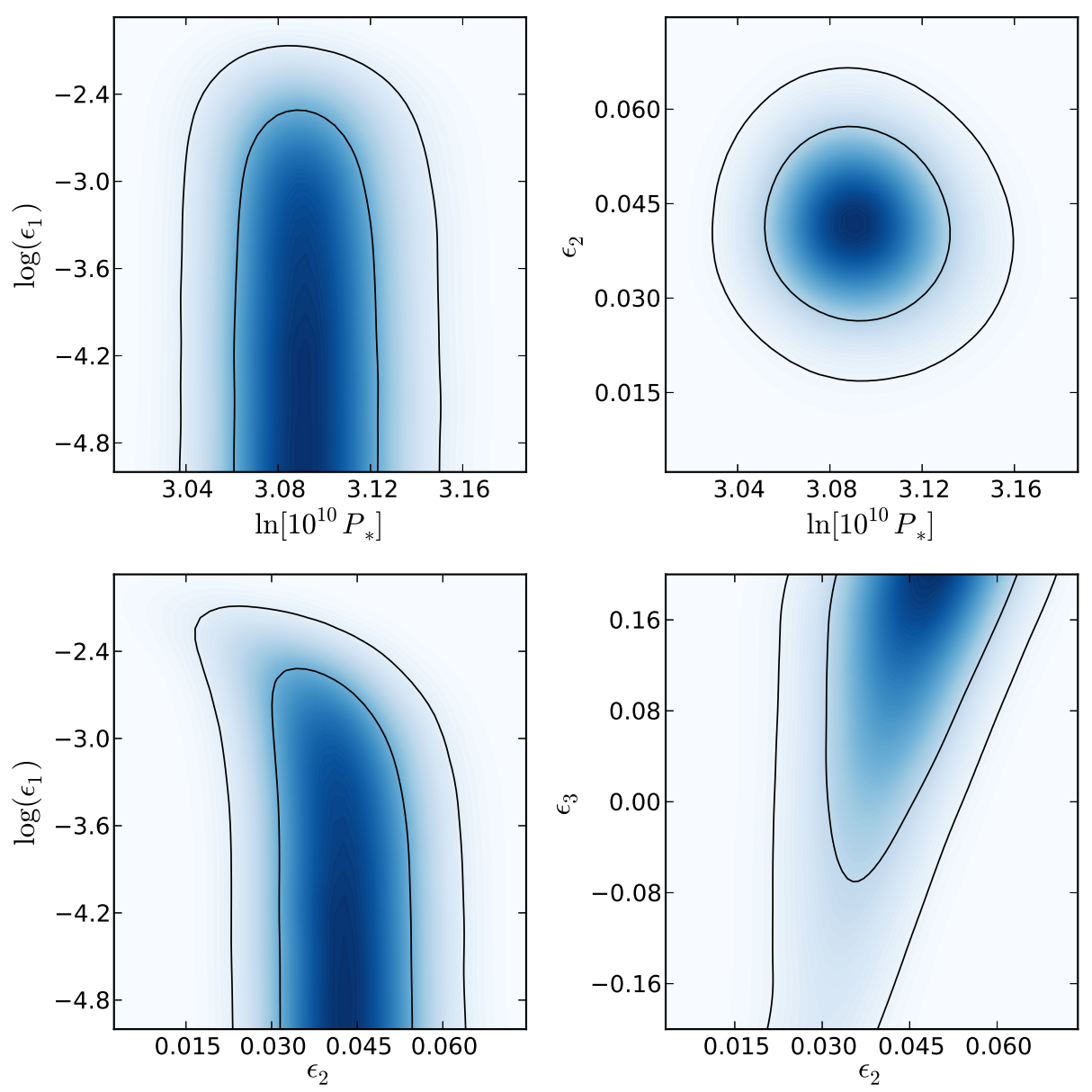

Figure 1. Two-dimensional marginalised posterior distributions of the slow-roll parameters $\left(P_{*}, \epsilon_{1}\right.$, $\left.\epsilon_{2}, \epsilon_{3}\right)$ using the Planck 2013 data.

Using the Planck likelihood and its associated public code CLIK, we have performed a MCMC exploration of the parameter space $\left(\theta_{\mathrm{s}}, P_{*}, \epsilon_{1}, \epsilon_{2}, \epsilon_{3}\right)$. In order to do so, we have used the public code COSMOMC [43] complemented by a modified version of the CAMB code [44] in order to implement as initial conditions the slow-roll primordial power spectra discussed above. All $\epsilon_{n}$ in these equations are evaluated at the conformal time $\eta_{*}$ defined by $k_{*} \eta_{*}=-1$, $k_{*}=0.05 \mathrm{Mpc}^{-1}$ being the pivot scale.

The prior choices for the parameters $\theta_{\mathrm{s}}$ have been chosen as in Ref.[1]. For the primordial parameter space, we have chosen a Jeffreys' prior for $P_{*}$ such that $\ln \left(10^{10} P_{*}\right) \in[2.7,4.2]$, i.e. centred around its well-measured value. The order of magnitude of the tensor-to-scalar ratio being unknown, we have chosen a wide Jeffreys' prior on $\epsilon_{1}$ as $\log \left(\epsilon_{1}\right) \in[-5,-0.7]$, the upper bound being such that $\epsilon_{1}<0.2$ to be within the slow-roll approximation. Finally, for $\epsilon_{2}$ and $\epsilon_{3}$ we have chosen uniform priors in $[-0.2,0.2]$. The MCMC exploration has been stopped once the total number of samples reached two millions, which corresponds to the $R$-statistics convergence of COSMOMC (the Gelman-Rubin criterion) to be less than $10^{-3}$ (see Ref. [43]). The thus obtained two-dimensional marginalised posterior probability distributions for the slow-roll parameters are shown in figure 1. More details on the analysis can be found in 
Ref. [6]. In particular, all the posteriors are compatible with those obtained by the Planck Collaboration in Refs. [1,3].

These MCMC samples have then be used to determine the effective likelihood for infla-

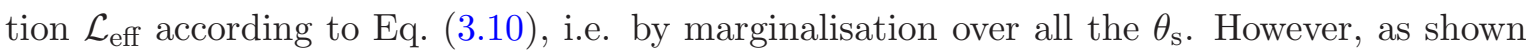
in figure $1, \epsilon_{3}$ is not well constrained. Therefore, following Ref. [6], it is more convenient to fit a three-dimensional likelihood $\mathcal{L}_{\text {eff }}\left(P_{*}, \epsilon_{1}, \epsilon_{2}\right)$ by additionally marginalising over $\epsilon_{3}$. Notice that doing so renders our analysis robust with respect to any uncertainties that are associated with the unconstrained second order terms. The fit itself have been implemented by a multivariate interpolation using a modified quadratic Shepard's method [45, 46]. Discussions on the method's accuracy can be found in Ref. [6] and we emphasise that the effective likelihood is only well approximated within the bounds $\ln \left(\mathcal{L}_{\text {eff }}^{\min } / \mathcal{L}_{\text {eff }}^{\max }\right)=-10$. Lower values of the likelihood have been extrapolated by assuming Gaussian tails. As a result, for a given model, the contribution to the Bayesian evidence from regions in parameter space where the likelihood is smaller than this value are not reliable. In practice, this is unlikely to be problematic because the contribution of regions with exceedingly small likelihood values to the evidence integral is minimal. Furthermore, models that never achieve a large value of the likelihood are in any case clearly ruled out, even though the value for their Bayesian evidence is only approximate.

Let us also stress that, for our purpose, $\mathcal{L}_{\text {eff }}\left(P_{*}, \epsilon_{1}, \epsilon_{2}\right)$ is now numerically known for any input values of $P_{*}, \epsilon_{1}$ and $\epsilon_{2}$ within the prior bounds mentioned earlier. As can be seen in the posterior of $\epsilon_{1}$ (see figure 1 ), $\mathcal{L}_{\text {eff }}$ has a flat direction for very small values of $\epsilon_{1}$. As a result, and only for $\epsilon_{1}, \mathcal{L}_{\text {eff }}$ has been extrapolated by a constant along its flat direction for $\log \left(\epsilon_{1}\right)<-5$, without loss of accuracy.

\subsection{Computing the evidences}

From the effective likelihood, and within a given model of inflation, we have used the nested sampling algorithm MultiNest [26, 27] to perform the multidimensional integral of Eq. (3.9). For each slow-roll scenario of the Encyclopæedia Inflationaris, the analytic form of the functionals $\epsilon_{n}\left(\theta_{\text {reh }}, \theta_{\text {inf }}\right)$ have been derived in Ref. [5] and they have been numerically evaluated using the public code ASPIC. The evidences reported below have been obtained by requiring a MultiNest target accuracy of $10^{-4}$ on the evidence and a number of live points equals to 30000. Typically, this amounts to a few hundred thousand samples for each model and around one hour of CPU time. We have not reported any numerical error on the evidences because, with such a target accuracy, they remain completely negligible with respect to the prior sensitivity effects.

Moreover, for all of the models, we have traded the parameter $M$, namely the mass scale giving the normalisation of the potential $V(\phi)$, by the amplitude $P_{*}$ of the scalar primordial power spectrum at the pivot wavenumber. Both of these parameters are indeed in one-to-one correspondence once the functionals $\epsilon_{n}\left(\theta_{\text {reh }}, \theta_{\text {inf }}\right)$ are given, but using $P_{*}$ instead of $M$ has the advantage of minimising superfluous degeneracies in the parameter space, as does the choice of using the rescaled parameter $R$ instead of $R_{\text {rad }}$. From the Friedmann-Lemaitre equation, one indeed has $[6]$

$$
M^{4}=24 \pi^{2} \frac{\epsilon_{1}}{v_{*}} P_{*},
$$

at first order in slow-roll. Here $v_{*} \equiv V\left(\phi_{*}\right) / M^{4}$, and $\phi_{*}=\phi\left(\eta_{*}\right)$.

These prior choices have important consequences for the evidence calculation. They imply that, for all models tested, the prior space on both the reheating, and the potential 
normalisation are the same. As a result, the Occam's razor factors for those parameters cancel out when computing the Bayes' factor between two models (this can be seen at once by employing the Savage-Dickey density ratio, see [14, 15]). In other words, we assume that all models have the same ability to reheat the universe after inflation and to produce the observed amplitude of the CMB anisotropies. As definite reheating predictions are almost absent in all the models we have explored, and those same models do not predict definite values of $M$, this is a fair assumption.

However, if one imagines a situation in which $M$ is an actual output of the model under scrutiny, its evidence should be reviewed. One may envisage two cases. Either the predicted values for $M$ (and $\epsilon_{1}$ ) yield a prior on $P_{*}$ whose support is outside the range we have used, i.e. $\ln \left(10^{10} P_{*}\right) \in[2.7,4.2]$ (see figure. 1 ), which is compatible with the data - in which case such a model would be ruled out; or it overlaps with it and the evidence should be recomputed by sampling the parameter space directly over $M$. In the situation for which the model's predictions for $M$ would actually match very well the observed amplitude of the CMB anisotropies, one should expect the Bayesian evidence of that precise model to be boosted in accordance with the Occam's razor principle. The same remarks hold concerning the reheating parameter [47]. Let us stress, however, that we have not encountered such a situation in all the models tested here.

\subsection{Fine-tuning issues}

For some of the models presented here, the slow-roll regime of inflation takes place only for a very limited range of values for some of their parameters. Such "fine-tuning" of parameters which have, a priori, no reason to take exactly such specific values, is disfavoured by the Occam's razor penalty in-built into the Bayesian evidence. From a technical point of view, the likelihood can reliably be worked out only in regimes where the slow-roll is (at least roughly) valid. Otherwise, the inflationary dynamics is very difficult to track and not described by our modelisation. On the other hand, when the slow-roll is completely violated, one knows that the associated predictions are ruled out by observations, and that the likelihood in this region of parameter space, being essentially 0 , does not contribute to the the total evidence. Therefore such situations result into an Occam's razor effect which suppresses the evidence computed over "compatible" parameters (the ones for which slow-roll inflation exist) by a factor equal to the ratio of the volume of compatible parameters over the whole prior volume. For the models in which this occurs, we have added some discussions in the appendix. 
Bayesian Evidences $\ln \left(\mathcal{E} / \mathcal{E}_{\mathrm{HI}}\right)$ and $\ln \left(\mathcal{L}_{\text {max }} / \mathcal{E}_{\mathrm{HI}}\right)$

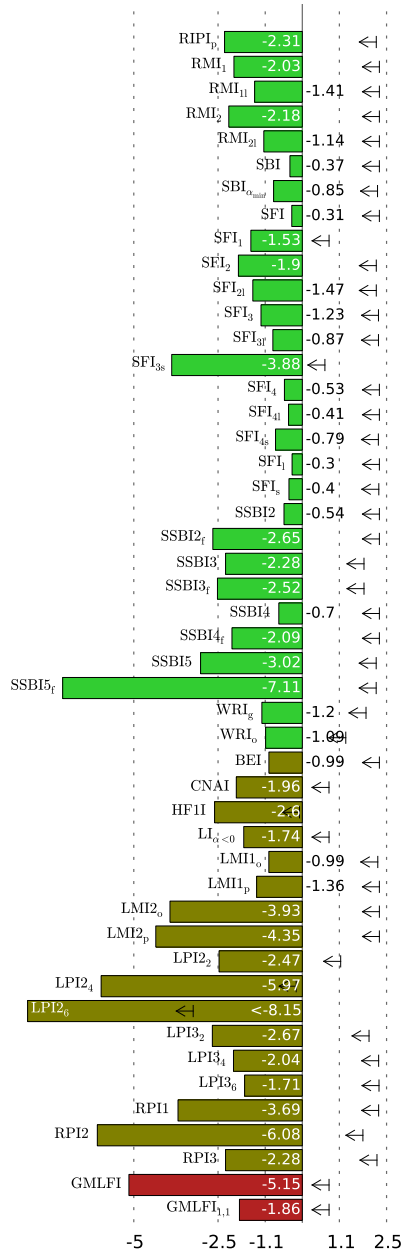

J.Martin, C.Ringeval, R.Trotta, V.Vennin

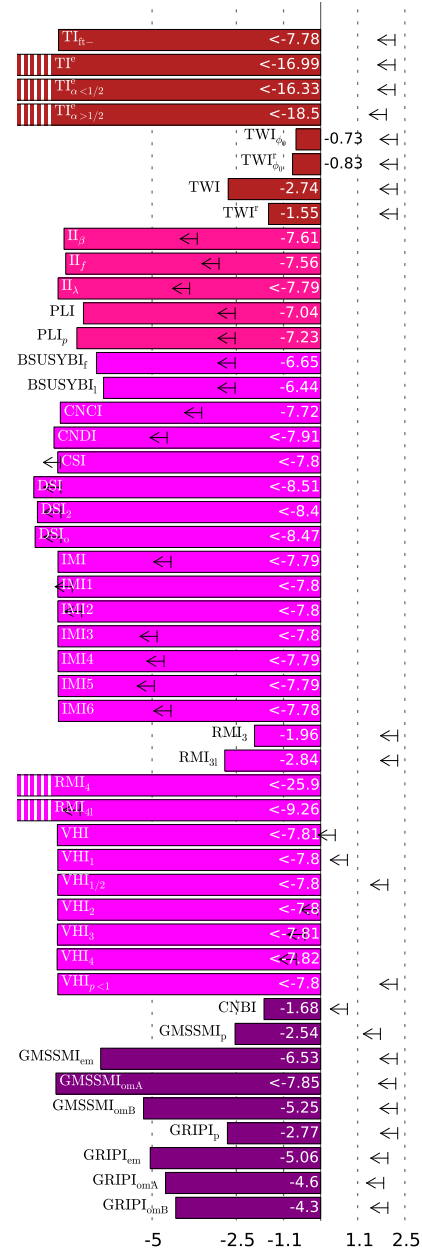

Displayed Evidences: 193

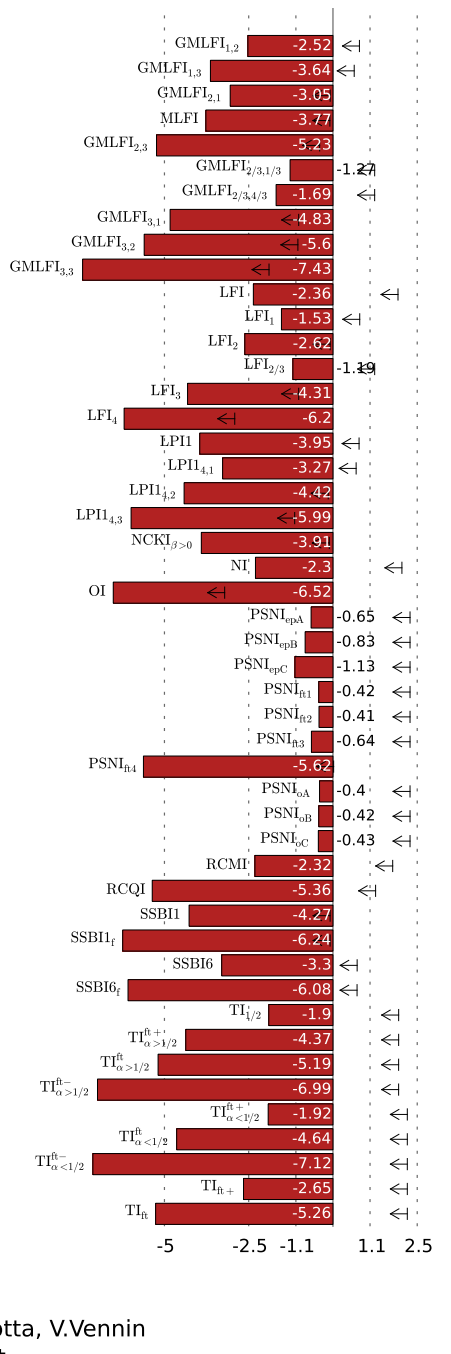

Figure 2. Bayes factors (bars) and absolute upper bound to the Bayes factors (arrows) for the Encyclopcedia Inflationaris inflationary scenarios, with Higgs inflation as the reference model (see the text for a more accurate description). 


\section{Results and discussion}

For all the models listed in the appendix A, i.e. $N^{\text {mod }}=193$, we have computed the Bayes factors $B_{\mathrm{HI}}^{i}$ with respect to the Starobinsky model [48-50] or Higgs Inflation (HI), which is our reference model. We have also evaluated each model's Bayesian complexity $\mathcal{C}_{i}$.

Our main results are displayed in figure 2, which represents all the Bayes factors. Each model is represented by a horizontal bar indicating the value of $\ln B_{\mathrm{HI}}^{i}$. A bar extending to the left corresponds to $\ln B_{\mathrm{HI}}^{i}<0$ and the model under consideration is disfavoured with respect to the the reference model. If, on the contrary, the bar extends to the right, then $\ln B_{\mathrm{HI}}^{i}>0$ and the model is preferred to Higgs inflation. Obviously, the Bayes factor of the reference model is one and, therefore, its logarithm vanishes: this is why there is no bar for HI. In front of (or inside) each bar, we have reported the exact numerical value of $\ln B_{\mathrm{HI}}^{i}$. We have also included the Jeffreys' scale of Table 1, as dashed vertical lines, as an indication of the viability of a given model compared to HI.

Bars are colour-coded according to the Schwarz-Terrero-Escalante (STE) classification associated with the slow-roll parameters of the model under consideration [40]. Following the notation used in Ref. [5], region 1 are models predicting $\epsilon_{2}>2 \epsilon_{1}>0$, i.e. the kinetic energy increases during inflation as well as the ratio of the kinetic energy to the total energy. Region 2 stands for potentials associated with $0<\epsilon_{2}<2 \epsilon_{1}$ for which the kinetic energy decreases while the ratio of the kinetic energy to the total energy still increases. Finally, region 3 is such that both quantities decrease during inflation. As shown in Ref. [5], the Planck 2013 results disfavour models living in regions 2 and 3 and the Bayes factors also reflect this. Let us stress that the parameter space of some models may span more than one region, i.e. for some values of its parameters the predictions of a model can fall in region 1 (say) while, for some other regime, they can be in region 2. It is referenced in the captions of figure 2 where the colour code takes this fact into account.

Finally, for each model, we have also calculated the maximum value of the evidence, that is to say the value that is obtained when all the prior mass for the model under consideration is concentrated in a delta-function centred at the maximum likelihood location. Clearly, in that case, one has $\mathcal{E}_{\max }=\mathcal{L}_{\max }$. It represents an absolute upper bound on the evidence: any choice of priors necessarily leads to a value of the evidence smaller than $\mathcal{E}_{\max }$. This upper bound is represented by black left-pointing arrows in figure 2. Let us also remark that this quantity would be relevant in a frequentist analysis where the $p$-value obtained from the maximum likelihood ratio would be used to compare the performances of different models.

Let us now analyse our results in more detail. Firstly, the answer to the central question of this paper, namely "what is the best model of inflation given the Planck 2013 data?" is KMIII inflation [51-53], whose Bayes factor with respect to Higgs inflation is $\ln B_{\mathrm{HI}}^{\mathrm{KMII}}=$ $0.07>0$. However, the preference is extremely mild, so much so that it is within the margin of uncertainty of our analysis, and for all practical purposes KMIII inflation has to be regarded as being on the same footing with Higgs inflation, from the point of view of the Planck data.

We can use the Jeffreys' scale as an indication for which of the models remain viable, and which are disfavoured at various levels of evidence with respect to the best models. We find 52 models in the "inconclusive" region (with respect to the best model), 41 in the "weakly disfavoured" region, 34 in the "moderately disfavoured" region and 66 in the "strongly disfavoured". Therefore, our analysis concludes that surviving models (i.e. those in the "inconclusive" region) represent $26 \%$ of the total. On the contrary, the number of models that are conclusively ruled out (i.e. those in the "strong" region) represent $34 \%$ of the total 
numbers of models. The models in the "inconclusive region", which are to be considered the best models of inflation after the Plank data, are (in alphabetical order ${ }^{4}$ ): $\mathrm{AI}, \mathrm{BI} \mathrm{ph}_{\mathrm{p}}, \mathrm{BI}_{\mathrm{s}}, \mathrm{BI}_{1 \mathrm{~s}}$, $\mathrm{BI}_{2 \mathrm{~s}}, \mathrm{BI}_{3 \mathrm{~s}}, \mathrm{BI}_{4 \mathrm{~s}}, \mathrm{BI}_{5 \mathrm{~s}}, \mathrm{BI}_{6 \mathrm{~s}}, \mathrm{BI}_{\mathrm{stg}}, \mathrm{ESI}, \mathrm{ESI}_{1}, \mathrm{ESI} \sqrt{2 / 3}, \mathrm{ESI}_{\sqrt{2}}, \mathrm{ESI}_{\mathrm{o}}, \mathrm{HI}, \mathrm{KKLTI}, \mathrm{KKLTI}_{\mathrm{s}}$, $\mathrm{KKLTI}_{\mathrm{stg}}, \mathrm{KMIII}_{\mathrm{T}} \mathrm{KMII}, \mathrm{KMII}_{V>0}$, LI, $\mathrm{LI}_{\alpha>0}, \mathrm{MHI}, \mathrm{MHI}_{\mathrm{l}}, \mathrm{MHI}_{\mathrm{s}}, \mathrm{PSNI}_{\mathrm{ft} 1}, \mathrm{PSNI}_{\mathrm{ft} 2}, \mathrm{PSNI}_{\mathrm{ft} 3}$, $\mathrm{PSNI}_{\mathrm{oA}}, \mathrm{PSNI}_{\mathrm{oB}}, \mathrm{PSNI}_{\mathrm{oC}}, \mathrm{PSNI}_{\mathrm{epA}}, \mathrm{PSNI}_{\mathrm{epB}}, \mathrm{RGI}, \mathrm{RGI}_{\mathrm{s}}, \mathrm{RGI}_{1}, \mathrm{RGI}_{1 / 16}, \mathrm{SBI}, \mathrm{SBI}_{\alpha_{\text {min }}}, \mathrm{SFI}$, $\mathrm{SFI}_{31}, \mathrm{SFI}_{4}, \mathrm{SFI}_{41}, \mathrm{SFI}_{4 \mathrm{~s}}, \mathrm{SFI}_{1}, \mathrm{SFI}_{\mathrm{s}}, \mathrm{SSBI} 2, \mathrm{SSBI} 4, \mathrm{TWI}_{\phi_{0}}$ and $\mathrm{TWI}_{\phi_{0}}^{\mathrm{r}}$. As explained above, there are more models than potential shapes because a given potential can support different priors, which are considered as separate model choices. As a consequence, the above 52 models in the "inconclusive region" encompass only 15 different potentials or scenarios.

Further insight can be garnered by considering the Bayesian complexity for each Encyclopadia Inflationaris model. We are particularly interested in evaluating the number of unconstrained parameters for the best models identified via the Bayesian evidence, i.e. the ones that are in the "inconclusive region". Since the Bayesian complexity measures the number of effective parameters supported by the data, one can define a measure of the number of unconstrained parameters by

$$
N_{i}^{\mathrm{uc}} \equiv N_{i}-\mathcal{C}_{i}
$$

where $N_{i}$ is the total number of free parameters of the model under consideration, i.e. the inflationary potential parameters, plus the reheating parameter. For models providing a reasonable good fit to the Planck data, one expects $N_{i}^{\text {uc }} \geq 0$. However, if the best-fit loglikelihood of a given model is very poor, then the Bayesian complexity can be arbitrary large, as the second term in Eq. (2.9) is large. This means that for such models $N_{i}^{\text {uc }}<0$. So we expect a negative measure of the number of unconstrained parameters to be correlated with a small value of the Bayes factor.

In figure 3 , we have plotted the location of all models in the two-dimensional plane $\left(N^{\mathrm{uc}}, \ln B_{\mathrm{HI}}\right)$. Models appearing along the same horizontal lines have thus the same Bayes factor but different number of unconstrained parameters $N^{\mathrm{uc}}$. Models with the smallest, non-negative number of unconstrained parameters are to be preferred in that they can be deemed to be simpler, even if they have the same evidence as other models with a larger value of $N^{\mathrm{uc}}$.

We can observe in figure 3 that models with $N^{\text {uc }}<0$ do have poor values of the evidence as well (ln $\left.B_{\mathrm{HI}}^{i} \ll 0\right)$, as expected from the above argument. Focusing on the models having the best evidences together with a minimal number of unconstrained parameters, i.e. $0<N_{i}^{\text {uc }}<1$ narrows down the slow-roll landscape to a few preferred models: $\mathrm{AI}, \mathrm{BI}_{1 \mathrm{~s}}, \mathrm{BI}_{2 \mathrm{~s}}$, $\mathrm{ESI}_{1}, \mathrm{ESI} \sqrt{2 / 3}, \mathrm{ESI}_{\sqrt{2}}, \mathrm{HI}, \mathrm{LI}_{\alpha>0}, \mathrm{MHI}, \mathrm{MHI}_{1}, \mathrm{RGI}, \mathrm{RGI}_{\mathrm{S}}, \mathrm{RGI}_{1}, \mathrm{SBI}_{\alpha_{\min }}, \mathrm{SFI}_{31}, \mathrm{SFI}_{4}$ and $\mathrm{SFI}_{41}$. We have now 17 preferred models, that is to say roughly $9 \%$ of the initial numbers of models. They correspond to only 9 types of potential or scenarios. It is also interesting to notice that KMIII is not in this set of preferred models since it has $N_{\mathrm{KMIII}}^{\mathrm{uc}} \simeq 2.3$. While it cannot be concluded that the models with the best Bayes factors and $0<N_{i}^{\text {uc }}<1$ are the "true" models, they are the simplest and most effective inflationary hypotheses that are compatible with the Planck 2013 CMB data. Obviously, allowing for more unconstrained parameters increases this list as displayed in figure 3.

Another interesting remark is that the 9 potentials mentioned above all belong to region 1 in the Schwarz-Terrero-Escalante classification (i.e. there are all "green"). This is of course consistent with the findings of Ref. [5] which has shown that this region is the region favoured

\footnotetext{
${ }^{4}$ The meaning of the different acronyms and the precise definition of the corresponding models can be found in appendix A.
} 

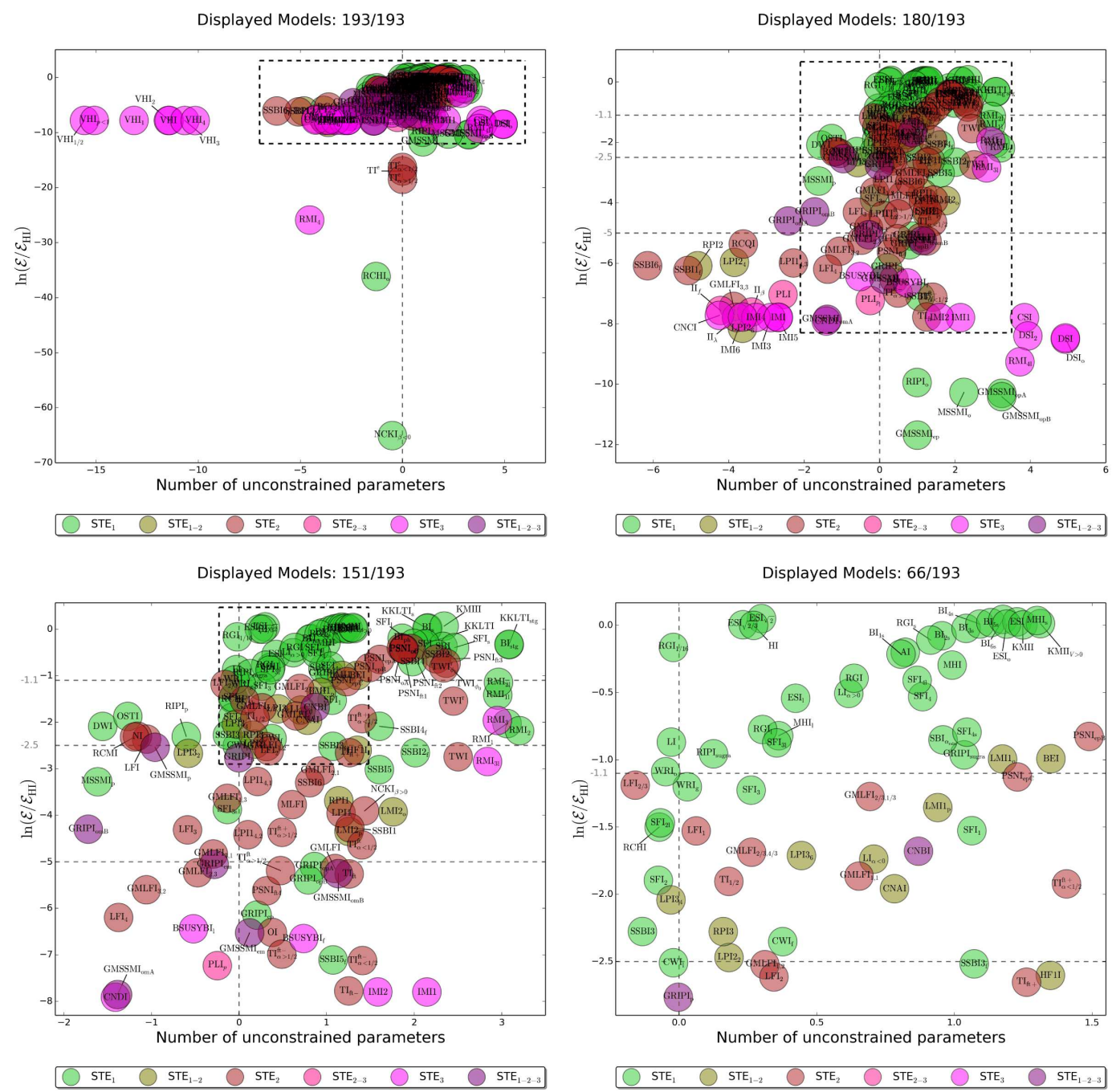

Figure 3. Logarithm of the Bayes factor versus the number of unconstrained parameters $N^{\text {uc }}$ for all the inflationary models investigated. The $N^{\mathrm{uc}}$ dimension allows us to disambiguate models with the same evidence, by preferring those with the smallest number of unconstrained (i.e., unnecessary) parameters. Optimal models are clustered around Higgs Inflation and have $N^{\mathrm{uc}} \simeq 0$ together with $B_{\mathrm{HI}} \gtrsim 0$. The four plots (from upper left to bottom right) increasingly zoom into the "best region". Each model is represented by a filled circle for illustration purposes only, and the radius of a circle has no meaning.

by the Planck data. This means that the corresponding models all belong to "plateau inflation" for which the potential does not necessarily grows to infinity when the vev of the field increases [54]. This type of potentials clearly appears to be the winners given the Planck data.

\section{Conclusions}

Let us now recap our main findings. Although this paper deals with slow-roll single-field inflation only, we do not expect multifield inflationary models to perform better than the 


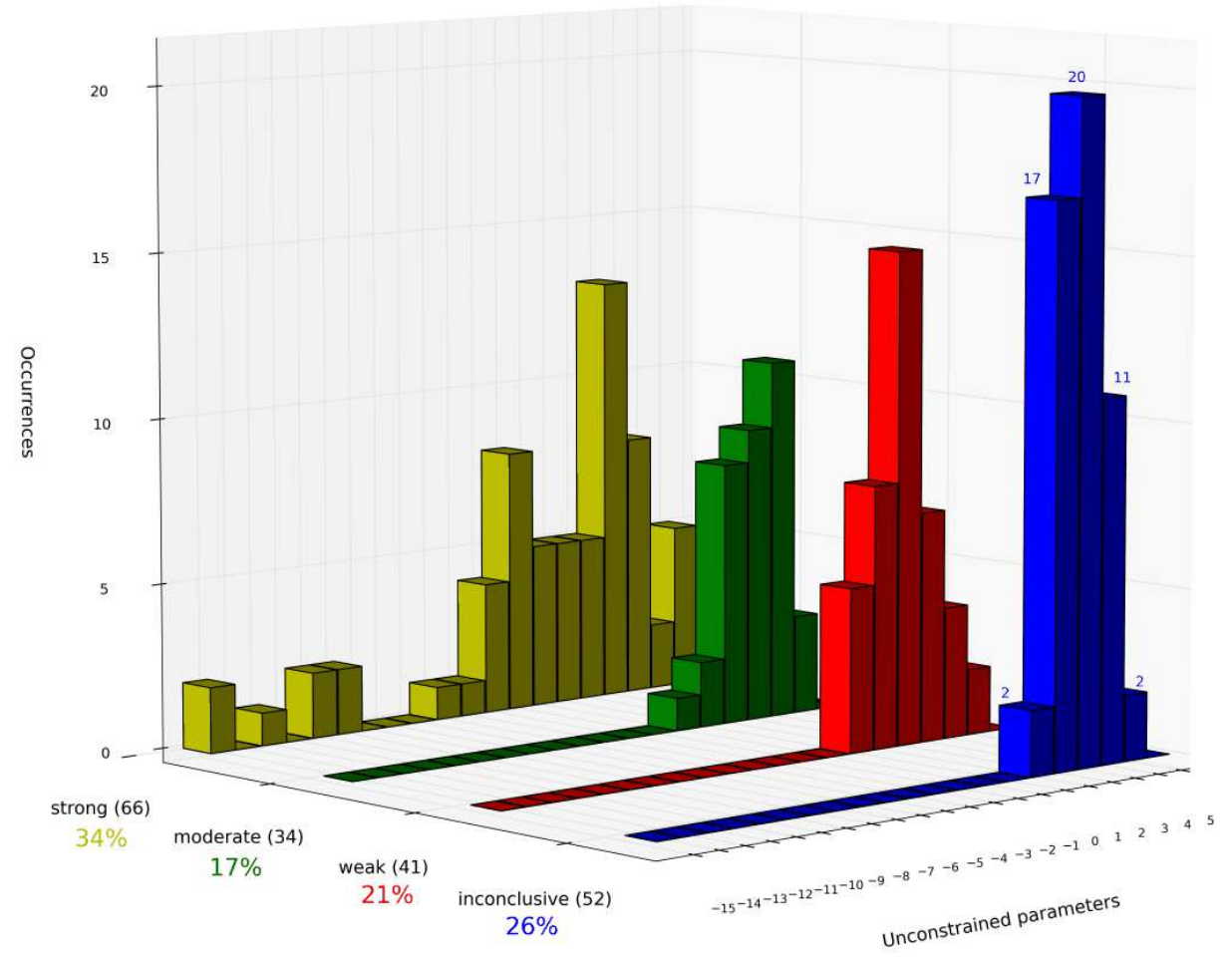

Figure 4. Histogram of the Encyclopadia Inflationaris models within the four Jeffreys' categories (inconclusive: blue, weakly disfavoured: red, moderately disfavoured: green and strongly disfavoured: yellow) and for different number of unconstrained parameters. The number of preferred models is 17 , corresponding to 9 different types of potential.

optimal subset of single-field models that have been delineated in this work. This is because adding a field necessarily introduces extra-parameters encoding the shape of the potential in this new direction. Therefore, even if a multifield scenario would fit as well the Planck 2013 data as the best slow-roll single-field models, such a model would be penalised by its larger number of unconstrained parameters (in terms of complexity). This conclusion could be modified if a multifields model was able to fit the large scales glitches in the Planck data, thus achieving a better evidence. However, those glitches are of relatively weak statistical significance and cannot, currently, greatly improve the overall fit. Furthermore, the fit improvement would have to be sufficient to offset the extra Occam's factor penalty implied by additional free parameters. Such a situation may however change by considering additional and independent data sets which could not be fitted by the class of slow-roll models discussed in this paper such as, for instance, a small, but non-vanishing, level of non-Gaussianities. The same remarks also apply for single-field scenarios with non-minimal kinetic terms (or with features in the potential). These models are not necessarily ruled out. However, either they predict observable non-Gaussianities and the fact that Planck sees a Gaussian sky implies that those models will be penalised for this wasted parameter space. Or, they genuinely do not predict non-Gaussianities but introduce additional parameters that increase the model complexity (see for instance Ref. [55-57]). Let us stress that, if we are not considering the small gain that might be associated with fitting Planck's glitches, the favoured models we have singled out in this paper already saturate the maximal possible value for the likelihood. 
As a result, even in the situation in which we would have missed an extremely good fitting and simple model, its Bayesian evidence would still be in the "inconclusive region".

Therefore, from a Bayesian point of view, it appears perfectly legitimate to focus on single-field slow-roll inflation (with a minimal kinetic term). These models have been studied and compared to the recent Planck data in Ref. [5] which, therefore, represents a complete cartography of the inflationary landscape compatible with the most recent data. In the present article, we have computed the Bayes factors and the Bayesian complexity for all these Encyclopadia Inflationaris models. Our results are summarised in an histogram in figure Fig. 4, which gives the number of models in each Jeffreys' category (defined with respect to the best model) and for each number of unconstrained parameters with $n<N^{\mathrm{uc}}<n+1$, where $n$ is an integer. This plot illustrates the power of the Planck data and allows us to summarise our main results: from a large number of models, one is able to single out a relatively small subset corresponding to the "best models". We rule out $\simeq 34 \%$ of the models at a strong level of evidence and $\simeq 26 \%$ of the models ( $9 \%$ if one includes the complexity) are preferred. All the favoured scenarios belong to the category 1 of the the Schwarz-TerreroEscalante classification and have a shape consistent with "plateau inflation".

It is also worth pointing out that a few Bayesian evidences have been calculated in Ref. [3]. The comparison is, however, difficult to carry out since the priors on reheating assumed in that paper greatly differ from those considered here ${ }^{5}$. Indeed, in Ref. [3], a prior on $\Delta N_{*}$ is chosen while the reheating energy density is arbitrarily fixed. There is no physical motivations for picking up particular values of the reheating energy density. Moreover, choosing a prior on $\Delta N_{*}$ is surprising since this does not guarantee the validity of the physical prior, namely $\rho_{\text {nuc }}<\rho_{\text {reh }}<\rho_{\text {end }}$. Another side effect is that this obviously modifies the calculation of the Bayesian evidences and, for this reason, comparing the two approaches does not lead to interesting insights.

To conclude this paper, let us present some speculations regarding what we have learnt about the physics of inflation. Firstly, let us stress that we have finally carried out one of the long standing task of primordial cosmology, namely put constraints on the shape of the inflationary potential. In some sense, this represents quite an impressive achievement since we are able to say something about physics at energy scales unreachable in accelerators. Indeed, with the Large Hardron Collider (LHC), it would obviously be impossible to establish the existence, at the Grand Unified Scale (GUT) scale, of a scalar field with a potential having a plateau shape. This perfectly illustrates the fact that cosmology can teach us something about high energy physics. On the other hand, this conclusion should be toned down: certainly, we have learnt a lot about the early Universe but, clearly, this does not give us the Lagrangian of particle physics at the GUT scales (i.e. the field content, their interactions etc ...). As a consequence, our knowledge of physics at such a high energy scale remains very limited. Hopefully, future analysis will help us to learn more about these questions. In this respect, constraining the reheating temperature of all the Encyclop cedia Inflationaris models seems promising since this can tell us something about the interaction of the inflaton field with the rest of the world.

Finally, one cannot help making the connection between the results obtained here and

\footnotetext{
${ }^{5}$ Let us also stress that the description made by Refs. [3] of the work of Ref. [29] on reheating is incorrect. It is claimed that the study of Ref. [29] is restricted to equations of state of the form $\bar{w}_{\text {reh }}=(p-2) /(p+2)$, which emerges in the case of a potential with the shape $\propto \phi^{p}$. This situation was indeed considered in Ref. [29] but only as a particular example. The completely generic case $-1 / 3<\bar{w}_{\text {reh }}<1$ was in fact the main concern of Ref. [29].
} 
the recent works about "conformal inflation" [49, 50, 58-66]. It is well-known that it is difficult to control the flatness of the inflaton potential that can easily be destroyed by quantum corrections. However, if one starts with any shape of $V(\phi)$, not necessarily very flat, and assumes a non-minimal coupling (for instance, of the form $\xi \phi^{2} R$ ), then, in the Einstein frame, the potential automatically flattens out and, precisely takes the form of plateau inflation for some range of the field. A striking example is provided in figure 4 of Ref. [61]: far from the origin, the potential automatically acquires the typical shape found in the present article to be favoured by the Planck data (see in particular right bottom of figure 4). Let us stress at this point that, although non-minimally coupled to gravity, this class of models belong to the ASPIC category since, after a conformal transformation to the Einstein frame, these models are in fact equivalent to single-field slow-roll inflation. In this representation, the non-triviality of the non-minimal coupling has been "transferred" to the complicated, non-minimal, interaction of $\phi$ with the other degrees of freedom present in the early Universe. In fact, Higgs inflation is the prototypical example of this class of scenarios and the ingredients necessary to describe the reheating phase in this case have been described in Ref. [5]. Therefore, we are in a situation where two strong theoretical arguments (the flatness of the potential and the presence of a non-minimal coupling to gravity - recalling that, according to the standard lore, a term that is not forbidden by a symmetry must be present in the theory) point precisely to the models that appear favoured by recent data. Whether this is just a coincidence or whether we are starting to understand something deeper about Nature will hopefully be answered in the near future when even more accurate data become available.

\section{Acknowledgments}

This work is partially supported by the ESA Belgian Federal PRODEX Grant No. 4000103071 and the Wallonia-Brussels Federation grant ARC No. 11/15-040.

\section{A Choice of priors for inflationary models}

In this appendix we detail the priors used in this article, and report the corresponding Bayesian evidences, complexities, number of parameters and likelihoods at the best fit point of all Encyclopadia Inflationaris scenarios. The priors are directly transcribed from considerations presented in Ref. [5], which is assumed to be known to the reader.

As discussed in section 2.2, there are cases where it is difficult to numerically estimate the evidences. In particular, this happens when one tries to extend the prior ranges in order to study the impact of the prior choices on our physical conclusions. However, most of the time, this prior sensitivity can be trivially accounted for by means of simple analytical calculations that we now briefly review. There are few instances in the following where they are concretely used.

A common situation is when the support of the likelihood is included in the prior range $\left[\theta_{\text {min }}, \theta_{\text {max }}\right]$, i.e. $\mathcal{L}(D \mid \theta, \mathcal{M}) \simeq 0$ for $\theta \notin\left[\theta_{\min }, \theta_{\text {max }}\right]$. The evidence of a model $\mathcal{M}$ is given by

$$
\mathcal{E}(D \mid \mathcal{M})=\int_{\theta_{\min }}^{\theta_{\max }} \mathrm{d} \theta \mathcal{L}(D \mid \theta, \mathcal{M}) \pi(\theta \mid \mathcal{M})
$$

where, for simplicity, we have assumed that there is only one parameter, $\theta$ (the argument can be generalised to any dimensions). For any proper (i.e., normalised) prior distribution 
$\pi(\theta \mid \mathcal{M})$, one has

$$
\pi(\theta \mid \mathcal{M})=\frac{\Pi(\theta)}{\int_{\theta_{\min }}^{\theta_{\max }} \mathrm{d} \theta \Pi(\theta)}, \quad \text { with } \quad \int_{\theta_{\min }}^{\theta_{\max }} \mathrm{d} \theta \pi(\theta \mid \mathcal{M})=1 .
$$

Let us assume that we change the prior range for the parameter $\theta$ and consider a new upper bound $\bar{\theta}_{\text {max }}$. The new prior is now given by

$$
\pi(\theta \mid \mathcal{M})=\frac{\Pi(\theta)}{\int_{\theta_{\min }}^{\bar{\theta}_{\max }} \mathrm{d} \theta \Pi(\theta)},
$$

where, in accordance with the above discussion, the likelihood is vanishing in $\left[\theta_{\max }, \bar{\theta}_{\max }\right]$. As a consequence, the value of the evidence for the larger prior range is given by

$$
\overline{\mathcal{E}}(D \mid \mathcal{M})=\int_{\theta_{\min }}^{\bar{\theta}_{\max }} \mathrm{d} \theta \mathcal{L}(D \mid \theta, \mathcal{M}) \pi(\theta \mid \mathcal{M})=\mathcal{E}(D \mid \mathcal{M}) \frac{\int_{\theta_{\min }}^{\theta_{\max }} \mathrm{d} \theta \Pi(\theta)}{\int_{\theta_{\min }}^{\bar{\theta}_{\max }} \mathrm{d} \theta \Pi(\theta)},
$$

and is obtained from the previous evidence value by simply rescaling it by the ratio of the prior volumes.

If instead the likelihood is flat along the $\theta$ direction, i.e. the data do not constrain the parameter under consideration, $\mathcal{L}(D \mid \theta, \mathcal{M})=\mathcal{L}_{0}$, then the evidence is unchanged by modifying the prior bounds

$$
\overline{\mathcal{E}}(D \mid \mathcal{M})=\int_{\theta_{\min }}^{\bar{\theta}_{\max }} \mathrm{d} \theta \mathcal{L}(D \mid \theta, \mathcal{M}) \pi(\theta \mid \mathcal{M})=\mathcal{L}_{0} \int_{\theta_{\min }}^{\bar{\theta}_{\max }} \mathrm{d} \theta \pi(\theta \mid \mathcal{M})=\mathcal{L}_{0}=\mathcal{E}(D \mid \mathcal{M}),
$$

and one should evaluate the Bayesian complexity to distinguish between the models.

Let us notice that the complexity may also be modified when the prior range is extended to regions where the likelihood is known to be negligible. However, contrary to the evidence, there is no simple analytical treatment of how the complexity should be extrapolated in this case. One can nevertheless make further simplifying assumptions to roughly estimate how the complexity is sensitive to the choice of priors.

Assuming that the prior and likelihood distributions are Gaussian, the complexity is given by $[23]$

$$
\mathcal{C}=\sum_{i=1}^{N} \frac{1}{1+\left(\frac{\sigma_{\mathcal{L}}^{i}}{\sigma_{\Pi}^{i}}\right)^{2}} \simeq \frac{N}{1+\left(\frac{\sigma_{\mathcal{L}}}{\sigma_{\Pi}}\right)^{2}}
$$

where $N$ is the number of parameters, $\sigma_{\Pi}^{i}$ and $\sigma_{\mathcal{L}}^{i}$ are the prior width and the standard deviations of the likelihood covariance matrix along its eigendirections $i$, respectively. The last approximation in the above equation assumes that one can define the averaged values $\sigma_{\Pi}$ and $\sigma_{\mathcal{L}}$ over all the eigendirections. If the prior is widened along $n$ directions (chosen among the $N$ parameters), its averaged volume $\sigma_{\Pi}^{n}$ gets multiplied by the same ratio $\overline{\mathcal{E}} / \mathcal{E}$ as computed above, i.e.

$$
\sigma_{\bar{\Pi}}=\sigma_{\Pi}\left(\frac{\mathcal{E}}{\overline{\mathcal{E}}}\right)^{\frac{1}{n}}
$$


Plugging back this relation in Eq. (A.6), one gets

$$
\overline{\mathcal{C}}=\frac{N}{1+\left(\frac{\overline{\mathcal{E}}}{\mathcal{E}}\right)^{2 / n}\left(\frac{N}{\mathcal{C}}-1\right)}
$$

where $\overline{\mathcal{E}} / \mathcal{E}$ is given by a volume ratio of the type Eq. (A.4).

In the next subsections, we discuss, for each Encyclopæedia Inflationaris scenarios, our choice of priors. We also give the definition of all the acronyms used in the paper, in particular in Fig. 2.

\section{A.1 Higgs Inflation (HI)}

The Higgs inflation model the potential of which is given by [5]

$$
V(\phi)=M^{4}\left(1-e^{-\sqrt{2 / 3} \phi / M_{\mathrm{P} 1}}\right)^{2},
$$

which contains only one parameter: the mass scale $M$. However, as discussed in section 3.3, this one has been traded for $P_{*}$ in our analysis and there are no other free parameter in this potential. In total, including the reheating parameter, one ends up with a two-parameters model. For this reason, and besides the fact that it was actually the first model of inflation ever proposed, we have chosen to take HI as the "reference model".

\begin{tabular}{|l||c||c||c||c||c|}
\hline Name & Priors & $\ln \left(\mathcal{E} / \mathcal{E}_{\mathrm{HI}}\right)$ & $\mathcal{C}$ & $N$ & $\ln \left(\mathcal{L}_{\max } / \mathcal{E}_{\mathrm{HI}}\right)$ \\
\hline HI & - & 0.00 & 1.73 & 2 & 2.22 \\
\hline
\end{tabular}

\section{A.2 Radiatively Corrected Higgs Inflation (RCHI)}

This model is a one-parameter model. The shape of the potential reads [5]

$$
V(\phi)=M^{4}\left(1-2 e^{-2 / \sqrt{6} \phi / M_{\mathrm{P} 1}}+\frac{A_{\mathrm{I}}}{16 \pi^{2}} \frac{\phi}{\sqrt{6} M_{\mathrm{P} 1}}\right) .
$$

The parameter $A_{\text {I }}$ controls the amplitude of the radiative corrections to the, tree level, HI potential. The one-loop expansion is valid under the condition $A_{\mathrm{I}} \ll 64 \pi^{2}$, hence the physical prior $A_{\mathrm{I}} \in[-100,100]$. However, numerically, when $A_{\mathrm{I}}<-65$, the likelihood is so small that it cannot be calculated in a reliable way. As a consequence, we choose the numerical prior to be $A_{\mathrm{I}} \in[-65,100]$. Anyhow, as already mentioned, the range $A_{\mathrm{I}} \in[-100,-65]$ does not contribute to the likelihood. On the other hand, as discussed in Ref. [5], particle physics implies $-48<A_{\mathrm{I}}<-20$ and this defines a new model, the "original" one, that we denote $\mathrm{RCHI}_{\mathrm{o}}$ in the following. We thus have two possible priors for this scenario as indicated by the following table:

\begin{tabular}{|l||c||c||c||c||c|}
\hline Name & Priors & $\ln \left(\mathcal{E} / \mathcal{E}_{\mathrm{HI}}\right)$ & $\mathcal{C}$ & $N$ & $\ln \left(\mathcal{L}_{\max } / \mathcal{E}_{\mathrm{HI}}\right)$ \\
\hline RCHI & $A_{\mathrm{I}} \in[-65,100]$ & -1.50 & 3.07 & 3 & 2.26 \\
RCHI $_{\mathrm{O}}$ & $A_{\mathrm{I}} \in[-48,-20]$ & -36.16 & 4.29 & 3 & -28.87 \\
\hline
\end{tabular}




\section{A.3 Large Field Inflation (LFI)}

Large field inflation is characterised by the following potential [5]

$$
V(\phi)=M^{4}\left(\frac{\phi}{M_{\mathrm{Pl}}}\right)^{p} .
$$

This potential depends on a mass scale $M$ fixed by the CMB normalisation and a free index $p$ of $\mathcal{O}(1)$ that can also take specific integer or rational values. Hence, one may assume a general prior on $p$ such that one can calculate the evidence of this class of model. Here one takes $p \in[0.2,5]$ because, for $p>5$, one already knows that the models are ruled out and $p>0.2$ instead of $p=0$ for numerical reasons (in addition, the potential cannot be completely flat since one needs to stop inflation). Another possibility is simply to fix $p$ to some interesting values: $p=2 / 3$ corresponds to monodromy inflation [67] while $p=1, \cdots, 4$ represents interesting phenomenological scenarios.

\begin{tabular}{|l||c||c||c||c||c|}
\hline Name & Priors & $\ln \left(\mathcal{E} / \mathcal{E}_{\mathrm{HI}}\right)$ & $\mathcal{C}$ & $N$ & $\ln \left(\mathcal{L}_{\max } / \mathcal{E}_{\mathrm{HI}}\right)$ \\
\hline $\mathrm{LFI}$ & $p \in[0.2,5]$ & -2.36 & 4.06 & 3 & 1.93 \\
$\mathrm{LFI}_{2 / 3}$ & $p=2 / 3$ & -1.19 & 2.16 & 2 & 1.24 \\
$\mathrm{LFI}_{1}$ & $p=1$ & -1.53 & 1.94 & 2 & 0.79 \\
$\mathrm{LFI}_{2}$ & $p=2$ & -2.62 & 1.66 & 2 & -0.08 \\
$\mathrm{LFI}_{3}$ & $p=3$ & -4.31 & 2.59 & 2 & -1.02 \\
$\mathrm{LFI}_{4}$ & $p=4$ & -6.20 & 3.38 & 2 & -2.91 \\
\hline
\end{tabular}

\section{A.4 Mixed Large Field Inflation (MLFI)}

This model possesses the following potential [5]

$$
V(\phi)=M^{4}\left(\frac{\phi}{M_{\mathrm{Pl}}}\right)^{2}\left(1+\alpha \frac{\phi^{2}}{M_{\mathrm{Pl}}^{2}}\right) .
$$

Beside the usual mass scale $M$ fixed by the CMB normalisation, MLFI contains only one parameter, $\alpha$. Since the order of magnitude of this parameter is a priori unknown, a Jeffreys prior on $\alpha$ is assumed. In practice, when $\alpha<10^{-5}$, the likelihood is numerically very close to that of $\mathrm{LFI}_{2}$ and when $\alpha>10$, the likelihood is numerically very close to that of $\mathrm{LFI}_{4}$. As a consequence, we take the prior given in the following table:

\begin{tabular}{|l||c||c||c||c||c|}
\hline Name & Priors & $\ln \left(\mathcal{E} / \mathcal{E}_{\mathrm{HI}}\right)$ & $\mathcal{C}$ & $N$ & $\ln \left(\mathcal{L}_{\max } / \mathcal{E}_{\mathrm{HI}}\right)$ \\
\hline MLFI & $\log (\alpha) \in[-5,1]$ & -3.77 & 2.39 & 3 & -0.09 \\
\hline
\end{tabular}

\section{A.5 Radiatively Corrected Massive Inflation (RCMI)}

The potential of this model is given by [5]

$$
V(\phi)=M^{4}\left(\frac{\phi}{M_{\mathrm{Pl}}}\right)^{2}\left[1-2 \alpha \frac{\phi^{2}}{M_{\mathrm{Pl}}^{2}} \ln \left(\frac{\phi}{M_{\mathrm{Pl}}}\right)\right] .
$$

It depends on one parameter, $\alpha$, which represents the amplitude of the radiative corrections to the potential of the $\mathrm{LFI}_{2}$ scenario. Since the one-loop correction can vary over many orders of magnitude, it is meaningful to choose a Jeffreys prior on $\alpha$. Then, clearly one must require $\alpha \ll 1$ in order for the perturbative expansion to be under control. On the other hand, the 
shape of the potential has been derived under the assumption that fermion loops dominate over self-interaction loops. This implies a lower bound on $\alpha$, namely $\alpha>10^{-15}$ [5]. However, when $\alpha<10^{-7}$, the likelihood is numerically very close to that of $\mathrm{LFI}_{2}$ and, therefore, it is not necessary to consider smaller values of $\alpha$. There also exists an upper bound on $\alpha$ coming from the requirement of having a sufficient number of e-folds during inflation, $\alpha<6 \times 10^{-4}$. Moreover, when $\alpha>10^{-3}$, the likelihood is so small that the evidence cannot be properly computed. As a consequence, an upper bound on $\alpha$ of $\simeq 10^{-3}$ seems to be an appropriate choice. Our choice is summarised in the following table:

\begin{tabular}{|l||c||c||c||c||c|}
\hline Name & Priors & $\ln \left(\mathcal{E} / \mathcal{E}_{\mathrm{HI}}\right)$ & $\mathcal{C}$ & $N$ & $\ln \left(\mathcal{L}_{\max } / \mathcal{E}_{\mathrm{HI}}\right)$ \\
\hline RCMI & $\log (\alpha) \in[-7,-3]$ & -2.32 & 4.19 & 3 & 1.77 \\
\hline
\end{tabular}

\section{A.6 Radiatively Corrected Quartic Inflation (RCQI)}

This model is a quartic large field model $\mathrm{LFI}_{4}$ plus radiative corrections [5]. The potential reads

$$
V(\phi)=M^{4}\left(\frac{\phi}{M_{\mathrm{Pl}}}\right)^{4}\left[1-\alpha \ln \left(\frac{\phi}{M_{\mathrm{Pl}}}\right)\right] .
$$

The amplitude of these corrections is controlled by the parameter $\alpha$. As discussed in the previous subsection, the order of magnitude of $\alpha$ is not known and, therefore, a Jeffreys prior must be chosen. Moreover, the perturbative expansion making sense only if the radiative correction is small, one must have $\alpha \ll 1$. The physical prior is therefore $\log (\alpha) \in[-\infty, 0]$. However, in practice, when $\alpha<10^{-3}$, the likelihood is numerically very close to that of $\mathrm{LFI}_{4}$ and when $\alpha>10^{-0.1}$, the likelihood is so small that it cannot be computed in a reliable way. Hence, the prior that we choose is the one indicated in the following table.

\begin{tabular}{|l||c||c||c||c||c|}
\hline Name & Priors & $\ln \left(\mathcal{E} / \mathcal{E}_{\mathrm{HI}}\right)$ & $\mathcal{C}$ & $N$ & $\ln \left(\mathcal{L}_{\max } / \mathcal{E}_{\mathrm{HI}}\right)$ \\
\hline RCQI & $\log (\alpha) \in[-3,-0.1]$ & -5.36 & 6.62 & 3 & 1.27 \\
\hline
\end{tabular}

\section{A.7 Natural Inflation (NI)}

This is a one parameter model and the potential is given by [5]

$$
V(\phi)=M^{4}\left[1+\cos \left(\frac{\phi}{f}\right)\right] .
$$

The order of magnitude of the free parameter $f$ is not known and, therefore, a Jeffreys prior is chosen. Moreover, the model is compatible with the CMB only if the mass scale $f$ is super-Planckian. It is not clear whether this condition makes sense at the fundamental level but, from the effective field point of view, several mechanisms have been invented such that this condition can be realised. In this situation $f$ can scale from a few $M_{\mathrm{Pl}}$ to $\sim 100 M_{\mathrm{Pl}}$, hence the prior $\log \left(f / M_{\mathrm{Pl}}\right) \in[0,2.5]$, see the following table.

\begin{tabular}{|l||c||c||c||c||c|}
\hline Name & Priors & $\ln \left(\mathcal{E} / \mathcal{E}_{\mathrm{HI}}\right)$ & $\mathcal{C}$ & $N$ & $\ln \left(\mathcal{L}_{\mathrm{max}} / \mathcal{E}_{\mathrm{HI}}\right)$ \\
\hline NI & $\log \left(f / M_{\mathrm{Pl}}\right) \in[0,2.5]$ & -2.30 & 4.16 & 3 & 2.05 \\
\hline
\end{tabular}




\section{A.8 Exponential SUSY Inflation (ESI)}

The potential of this model can be written as [5]

$$
V(\phi)=M^{4}\left(1-e^{-q \phi / M_{\mathrm{Pl}}}\right),
$$

where $q$ is a free parameter. A priori, different priors on $q$ are possible and this gives rise to different versions of this scenario. If we view ESI as a phenomenological model, then one can assume that the parameter $q$ is a free $\mathcal{O}(1)$ quantity. In that case, a natural prior is $q \in[0.1,6]$. But one can also assume that the order of magnitude of $q$ is not known (in the following, we denote the corresponding version of the scenario by $\mathrm{ESI}_{1}$ ). In this situation, we must choose a Jeffreys prior, typically $\log (q) \in[-3,3]$. However, when $q>1$, the model is numerically difficult to track since it produces a too weak level of gravity waves. Moreover, in this regime, the likelihood reaches a stationary value. Therefore, as explained before, one can restrict ourselves to the numerical prior $\log (q) \in[-3,1]$.

Another possible prior is based on the original derivation of the ESI scenario (we denote this version by $\mathrm{ESI}_{\mathrm{O}}$ in what follows). Indeed, in that case, the model is based on supergravity and one has $q=\sqrt{2 / \beta}$, where $\beta$ is the coefficient which appears in front of the Kähler potential of the model. Hence, it seems reasonable to assume that this quantity is a coefficient of order one. This justifies our choice for the "original" prior, namely $\beta \in[1,4]$. Of course, specific values of $\beta$ are also very relevant. In particular, $\beta=1$ or $\beta=3$ represents the cases where the inflaton field is either a dilaton or a moduli $(\beta=3$ corresponds to the "no scale" structure). In the following, we denote these versions of the ESI scenario by ESI $\sqrt{2}$ and ESI $\sqrt{2 / 3}$, respectively.

\begin{tabular}{|l||c||c||c||c||c|}
\hline Name & Priors & $\ln \left(\mathcal{E} / \mathcal{E}_{\mathrm{HI}}\right)$ & $\mathcal{C}$ & $N$ & $\ln \left(\mathcal{L}_{\mathrm{max}} / \mathcal{E}_{\mathrm{HI}}\right)$ \\
\hline ESI $_{\text {ESI }_{1}}$ & $q \in[0.1,6]$ & 0.03 & 1.77 & 3 & 2.29 \\
ESI $_{\mathrm{O}}$ & $\beta=2 / q^{2} \in[-3,1]$ & -0.54 & 2.58 & 3 & 2.29 \\
ESI $_{\sqrt{2}}$ & $q=\sqrt{2}$ & 0.01 & 1.81 & 3 & 2.25 \\
ESI $_{\sqrt{2 / 3}}$ & $q=\sqrt{2 / 3}$ & 0.05 & 1.70 & 2 & 2.25 \\
\end{tabular}

\section{A.9 Power Law Inflation (PLI)}

The potential of this class of models can be expressed as [5]

$$
V(\phi)=M^{4} e^{-\alpha \phi / M_{\mathrm{P} 1}},
$$

where $\alpha$ is a positive coefficient. A priori, it is a small quantity the order of magnitude of which is not known. As a consequence, a Jeffreys prior seems to be the most natural choice and we take $\log (\alpha) \in[-4,0]$. On a more phenomenological viewpoint, inflation occurs when $\alpha<\sqrt{2}$ only and, therefore, it makes also sense to choose a flat prior on $\alpha$, namely $\alpha \in[0, \sqrt{2}]$ (in the following, we denote this version of power law inflation by $\mathrm{PLI}_{p}$ ). However, when $\alpha>1.1$, the likelihood is so small that it cannot be properly calculated. Hence, we will restrict ourselves to the prior $\alpha \in[0,1.1]$.

\begin{tabular}{|l||c||c||c||c||c|}
\hline Name & Priors & $\ln \left(\mathcal{E} / \mathcal{E}_{\mathrm{HI}}\right)$ & $\mathcal{C}$ & $N$ & $\ln \left(\mathcal{L}_{\max } / \mathcal{E}_{\mathrm{HI}}\right)$ \\
\hline PLI & $\log (\alpha) \in[-4,0]$ & -7.04 & 5.56 & 3 & -2.54 \\
PLI $_{p}$ & $\alpha \in[0,1.1]$ & -7.23 & 3.25 & 3 & -2.54 \\
\hline
\end{tabular}




\section{A.10 Kähler Moduli Inflation (KMII)}

The potential of KMII inflation is given by [5]

$$
V(\phi)=M^{4}\left(1-\alpha \frac{\phi}{M_{\mathrm{P} 1}} e^{-\phi / M_{\mathrm{P} 1}}\right),
$$

where $\alpha$ is a free positive coefficient. As discussed in detail in Ref. [5], in order for inflation to end by slow-roll violation, one must have $\alpha \gtrsim 2.4095$. On the other hand, the order of magnitude of this parameter is unspecified and this suggests a Jeffreys prior on $\alpha$. Combining these two pieces of information, we are led to the prior $\log (\alpha) \in[\log (2.4095) \simeq 0.382,4]$.

On the other hand, one can also choose $\alpha$ such that the potential is positive everywhere, as opposed to the previous situation where, for some values of the field, the potential can be negative and where one makes use of a finite portion of it only (the corresponding version of the scenario is denoted by $\mathrm{KMII}_{V>0}$ in the following). In that case, one has the extra condition $\alpha<e \simeq 2.7183$. Since $e$ is close to 2.4095, a Jeffreys prior no longer makes sense and a linear prior now seems a sensible choice. Hence our second choice $\alpha \in[2.4095, e \simeq 2.7183]$. Everything is summarised in the following table:

\begin{tabular}{|l||c||c||c||c||c|}
\hline Name & Priors & $\ln \left(\mathcal{E} / \mathcal{E}_{\mathrm{HI}}\right)$ & $\mathcal{C}$ & $N$ & $\ln \left(\mathcal{L}_{\max } / \mathcal{E}_{\mathrm{HI}}\right)$ \\
\hline KMII $_{\mathrm{KMII}_{V>0}}$ & $\log (\alpha) \in[0.382,4]$ & 0.00 & 1.75 & 3 & 2.22 \\
& $\alpha \in[2.4095,2.7183]$ & 0.01 & 1.69 & 3 & 2.22 \\
\hline
\end{tabular}

\section{A.11 Horizon Flow Inflation at first order (HF1I)}

The potential of HF1I inflation reads [5]

$$
V(\phi)=M^{4}\left(1+A_{1} \frac{\phi}{M_{\mathrm{Pl}}}\right)^{2}\left[1-\frac{2}{3}\left(\frac{A_{1}}{1+A_{1} \phi / M_{\mathrm{Pl}}}\right)^{2}\right] .
$$

This model is obtained by an integration of the horizon flow equations truncated at a given order (here at second order). As such, this scenario is in fact purely phenomenological. Moreover, it turns out that the observational predictions are not very sensitive to the value of the free parameter $A_{1}$. Therefore, since its order of magnitude is not fixed, it makes sense to choose a Jeffrey prior on $A_{1}$ and we take $\log \left(A_{1}\right) \in[-3,3]$ as indicated below.

\begin{tabular}{|l||c||c||c||c||c|}
\hline Name & Priors & $\ln \left(\mathcal{E} / \mathcal{E}_{\mathrm{HI}}\right)$ & $\mathcal{C}$ & $N$ & $\ln \left(\mathcal{L}_{\max } / \mathcal{E}_{\mathrm{HI}}\right)$ \\
\hline HF1I & $\log \left(A_{1}\right) \in[-3,3]$ & -2.60 & 1.65 & 3 & -0.08 \\
\hline
\end{tabular}

\section{A.12 Coleman Weinberg Inflation (CWI)}

Coleman Weinberg inflation is based on the following potential [5]

$$
V(\phi)=M^{4}\left[1+\alpha\left(\frac{\phi}{Q}\right)^{4} \ln \left(\frac{\phi}{Q}\right)\right]
$$

with $\alpha=4 e$ in order to have a vanishing minimum. The shape of $V(\phi)$ is therefore characterised by only one parameter, $Q$. In the original version of the scenario, $Q$ is fixed by the GUT scale, $Q \sim 10^{14}-10^{15} \mathrm{GeV}$. Therefore, in this case, it is natural to choose a flat prior on $Q$ (we denote this version of the scenario by $\mathrm{CWI}_{\mathrm{f}}$ ). On the other hand, if one considers a more general situation, then there is a priori no criterion to fix the value (or the order of magnitude) of $Q$ and, therefore, this justifies the choice of a Jeffreys prior, namely $\log \left(Q / M_{\mathrm{Pl}}\right) \in[-5,-3]$ (we denote the version of the scenario by $\mathrm{CWI}_{1}$ ). 


\begin{tabular}{|c||c||c||c||c||c|}
\hline Name & Priors & $\ln \left(\mathcal{E} / \mathcal{E}_{\mathrm{HI}}\right)$ & $\mathcal{C}$ & $N$ & $\ln \left(\mathcal{L}_{\max } / \mathcal{E}_{\mathrm{HI}}\right)$ \\
\hline $\mathrm{CWI}_{\mathrm{f}}$ & $Q / M_{\mathrm{Pl}} \in\left[5 \times 10^{-5}, 5 \times 10^{-4}\right]$ & -2.35 & 2.62 & 3 & 1.49 \\
$\mathrm{CWI}_{\mathrm{l}}$ & $\log \left(Q / M_{\mathrm{Pl}}\right) \in[-5,-3]$ & -2.51 & 3.02 & 3 & 1.60 \\
\hline
\end{tabular}

\section{A.13 Loop Inflation (LI)}

The potential of LI inflation can be written as [5]

$$
V(\phi)=M^{4}\left[1+\alpha \ln \left(\frac{\phi}{M_{\mathrm{Pl}}}\right)\right],
$$

where the parameter $\alpha$ controls the strength of the one loop correction to the tree level $V(\phi)$ (here the constant term) and must therefore be such that $\alpha \ll 1$. When $\alpha<0$, in order to have a sufficient number of $e$-folds, one must require $\alpha>\alpha_{\min } \simeq-0.3$ [5]. In principle, the model makes sense only if inflation proceeds at sub-Planckian vev's which is, strictly speaking, not possible in this regime. If we allow vev's larger than the Planck mass, typically up to $\phi / M_{\mathrm{Pl}} \simeq 1000$, then this sets an additional condition, namely $\alpha<-0.1$. When $\alpha>0$, there is no extra condition on $\alpha$ except, as already signaled, that $\alpha$ must be small in order for the perturbative expansion to make sense.

From the previous considerations, we assume a flat prior $\alpha \in[-0.3,-0.1]$ in the case where $\alpha<0$ (we denote this version of the scenario by $\mathrm{LI}_{\alpha<0}$ ). We have seen that, when $\alpha>0$, there exists no restrictions on this parameter. In particular, its order of magnitude is not specified and, therefore, it makes sense to choose a Jeffreys prior, namely $\log (\alpha) \in$ $[\log (0.003), \log (0.3)]$ (in the following, this version of the scenario is denoted by $\mathrm{LI}_{\alpha>0}$ ). Finally, when the sign is left unspecified, we simply consider a flat prior $\alpha \in\left[\alpha_{\min },-0.1\right] \cup$ $[0,0.3]$. These priors are summarised in the following table.

\begin{tabular}{|l||c||c||c||c||c|}
\hline Name & Priors & $\ln \left(\mathcal{E} / \mathcal{E}_{\mathrm{HI}}\right)$ & $\mathcal{C}$ & $N$ & $\ln \left(\mathcal{L}_{\mathrm{max}} / \mathcal{E}_{\mathrm{HI}}\right)$ \\
\hline $\mathrm{LI}$ & $\alpha \in\left[\alpha_{\min },-0.1\right] \cup[0,0.3]$ & -0.87 & 3.03 & 3 & 2.27 \\
$\mathrm{LI}_{\alpha>0}$ & $\log (\alpha) \in[\log (0.003), \log (0.3)]$ & -0.51 & 2.38 & 3 & 2.27 \\
$\mathrm{LI}_{\alpha<0}$ & $\alpha \in\left[\alpha_{\min },-0.1\right]$ & -1.74 & 2.29 & 3 & 0.79 \\
\hline
\end{tabular}

\section{A.14 $R+R^{2 p}$ Inflation ( RpI)}

The potential of $R+R^{2 p}$ inflation can be expressed as

$$
V(\phi)=M^{4} e^{-2 \sqrt{2 / 3} \phi / M_{\mathrm{Pl}}}\left|e^{\sqrt{2 / 3} \phi / M_{\mathrm{Pl}}}-1\right|^{2 p /(2 p-1)},
$$

which depends on the parameter $p$. The case $p=1$ is peculiar and corresponds to Higgs Inflation (HI). It has been shown in Ref. [5] that, if $p$ takes integer values different from $p=1$, then the model is ruled out since it leads to values of $r$ and $n_{\mathrm{S}}$ that are not compatible with the Planck data. As a consequence, $p$ must be sufficiently close to 1 , and therefore must be a real number. When $p>1$, the potential possesses a maximum located at

$$
\frac{\phi_{\max }}{M_{\mathrm{P} 1}}=\sqrt{\frac{3}{2}} \ln \left(\frac{2 p-1}{p-1}\right) .
$$

and two regimes of inflation exist (denoted by RPI1 and RPI2 in what follows) depending on whether inflation takes place in $\phi \in\left[0, \phi_{\max }\right]$ or in $\phi \in\left[\phi_{\max }, \infty\right]$. In the first case, inflation stops by slow-roll violation and the model is therefore a one parameter model. In the second 
case, however, inflation must stop by instability at $\phi_{\text {end }}$ and, hence, the corresponding model is in fact a two parameters model, $p$ and $\phi_{\text {end }}$. Since $p$ must be close to one, we choose the flat prior $p \in[1,1.5]$. In the case of RPI2, the order of magnitude of $\phi_{\text {end }}$ being unspecified, we take the following Jeffreys prior on $\phi_{\text {end }}: \log \left(\phi_{\text {end }} / \phi_{\max }\right) \in[0.5,2]$.

If $p<1$, then there is a single regime where inflation can proceed. It is denoted by RPI3 in what follows. In that case, inflation stops by violation of the slow-roll conditions and, therefore, the model is a one parameter model. As a consequence, we choose to consider the following flat prior on $p: p \in[0.8,1]$.

\begin{tabular}{|l||c||c||c||c||c|}
\hline Name & Priors & $\ln \left(\mathcal{E} / \mathcal{E}_{\mathrm{HI}}\right)$ & $\mathcal{C}$ & $N$ & $\ln \left(\mathcal{L}_{\max } / \mathcal{E}_{\mathrm{HI}}\right)$ \\
\hline RPI1 & $p \in[1,1.5]$ & -3.69 & 1.86 & 3 & 2.26 \\
\hline RPI2 & $\begin{array}{c}p \in[1,1.5] \\
\log \left(\phi_{\text {end }} / \phi_{\max }\right) \in[0.8,1]\end{array}$ & -6.08 & 8.82 & 4 & 1.80 \\
\hline RPI3 & $p \in[0.8,1]$ & -2.28 & 2.84 & 3 & 2.22 \\
\hline
\end{tabular}

\section{A.15 Double Well Inflation (DWI)}

Double Well inflation is a one parameter model characterised by the following potential

$$
V(\phi)=M^{4}\left[\left(\frac{\phi}{\phi_{0}}\right)^{2}-1\right]^{2} .
$$

As shown in Ref. [5], slow-roll inflation takes place only if $\phi_{0} / M_{\mathrm{P} 1}>2 \sqrt{2}$. On the other hand, COBE normalising the model allows us to express the mass scale $M$ in terms of the free parameter $\phi_{0}$. Then, the requirement $M / M_{\mathrm{P} 1}<1$ leads to to the constraint $\phi_{0} / M_{\mathrm{P} 1} \lesssim 10^{5}$. As a consequence, a Jeffreys logarithmic prior on $\phi_{0}$ is chosen, namely $\log \left(\phi_{0} / M_{\mathrm{Pl}}\right) \in[\log (2 \sqrt{2}) \simeq$ $0.45,5]$.

\begin{tabular}{|l||c||c||c||c||c|}
\hline Name & Priors & $\ln \left(\mathcal{E} / \mathcal{E}_{\mathrm{HI}}\right)$ & $\mathcal{C}$ & $N$ & $\ln \left(\mathcal{L}_{\max } / \mathcal{E}_{\mathrm{HI}}\right)$ \\
\hline DWI & $\log \left(\phi_{0} / M_{\mathrm{Pl}}\right) \in[\log (2 \sqrt{2}), 5]$ & -2.09 & 4.56 & 3 & 2.14 \\
\hline
\end{tabular}

\section{A.16 Mutated Hilltop Inflation (MHI)}

The potential of Mutated Hilltop inflation is given by

$$
V(\phi)=M^{4}\left[1-\operatorname{sech}\left(\frac{\phi}{\mu}\right)\right]
$$

and depends on one free parameter, $\mu$. This model is phenomenological although it is supposed to emerge from supergravity considerations. In this last case, only sub-Planckian values for $\mu$ probably make sense. This is the reason why it seems interesting to consider different priors. Given that the order of magnitude of $\mu / M_{\mathrm{P} 1}$ is not specified, we take three Jeffreys priors corresponding to situations where $\mu$ is sub-Planckian (denoted by $\mathrm{MHI}_{1}$ ), super-Planckian (denoted by $\mathrm{MHI}_{\mathrm{s}}$ ) or not specified. Those choices are summarised in the following table:

\begin{tabular}{|l||c||c||c||c||c|}
\hline Name & Priors & $\ln \left(\mathcal{E} / \mathcal{E}_{\mathrm{HI}}\right)$ & $\mathcal{C}$ & $N$ & $\ln \left(\mathcal{L}_{\mathrm{max}} / \mathcal{E}_{\mathrm{HI}}\right)$ \\
\hline $\mathrm{MHI}$ & $\log \left(\mu / M_{\mathrm{Pl}}\right) \in[-2,2]$ & -0.30 & 2.01 & 3 & 2.29 \\
$\mathrm{MHI}_{\mathrm{l}}$ & $\log \left(\mu / M_{\mathrm{Pl}}\right) \in[-2,0]$ & -0.82 & 2.64 & 3 & 2.23 \\
$\mathrm{MHI}_{\mathrm{S}}$ & $\log \left(\mu / M_{\mathrm{Pl}}\right) \in[0,2]$ & 0.04 & 1.70 & 3 & 2.29 \\
\hline
\end{tabular}




\section{A.17 Radion Gauge Inflation (RGI)}

The potential of Radion Gauge inflation can be expressed as

$$
V(\phi)=M^{4} \frac{\left(\phi / M_{\mathrm{Pl}}\right)^{2}}{\alpha+\left(\phi / M_{\mathrm{Pl}}\right)^{2}},
$$

where $\alpha$ is a dimensionless positive parameter. A priori, smaller than unity values are preferred but, at the same time, $\alpha>1$ is not forbidden. This is why it is interesting to study how the Bayesian evidence of the model depends on the range of variation of $\alpha$. Let us also notice that the order of magnitude of this parameter is not specified. As a consequence, we choose three Jeffreys priors, one such that $\log (\alpha) \in[-4,4]$, one corresponding to a situation where $\alpha<1$, namely $\log (\alpha) \in[-4,0]$ (and we denote this version of the model by $\mathrm{RGI}_{\mathrm{s}}$ ) and one corresponding to $\alpha>1$, namely $\log (\alpha) \in[0,4]$ (this version being referred to as $\mathrm{RGI}_{1}$ ). Finally, in Ref. [68], the potential of Radion Gauge inflation was also obtained in the context of S-dual superstring models. In that case, the value of $\alpha$ is fixed and given by $\alpha=1 / 16$ which leads to a fourth choice of prior. Everything is summarised in the following table:

\begin{tabular}{|l||c||c||c||c||c|}
\hline Name & Priors & $\ln \left(\mathcal{E} / \mathcal{E}_{\mathrm{HI}}\right)$ & $\mathcal{C}$ & $N$ & $\ln \left(\mathcal{L}_{\max } / \mathcal{E}_{\mathrm{HI}}\right)$ \\
\hline RGI & $\log (\alpha) \in[-4,4]$ & -0.39 & 2.36 & 3 & 2.29 \\
$\mathrm{RGI}_{\mathrm{S}}$ & $\log (\alpha) \in[-4,0]$ & -0.11 & 2.09 & 3 & 2.29 \\
$\mathrm{RGI}_{1}$ & $\log (\alpha) \in[0,4]$ & -0.77 & 2.70 & 3 & 2.20 \\
RGI $_{1 / 16}$ & $\alpha=1 / 16$ & -0.16 & 2.02 & 2 & 2.20 \\
\hline
\end{tabular}

\section{A.18 MSSM Inflation (MSSMI)}

In this scenario, inflation occurs along a flat direction of the MSSM potential. This flat direction is usually lifted by higher order non-renormalisable operators and SUSY soft terms. As a consequence, one can show that the potential takes the form [5]

$$
V(\phi)=M^{4}\left[\left(\frac{\phi}{\phi_{0}}\right)^{2}-\frac{2}{3}\left(\frac{\phi}{\phi_{0}}\right)^{6}+\frac{1}{5}\left(\frac{\phi}{\phi_{0}}\right)^{10}\right]
$$

where $\phi_{0}$ is a free parameter which can be expressed as

$$
\phi_{0}^{8}=\frac{M_{\mathrm{P} 1}^{6} m_{\phi}^{2}}{10 \lambda_{6}^{2}} .
$$

The quantity $\lambda_{6}$ is a coupling constant that is taken to be of order one while $m_{\phi}$ is a soft breaking mass and, thus, is chosen to be around $\simeq 1 \mathrm{TeV}$. As a consequence, one has $\phi_{0} \simeq 10^{14} \mathrm{GeV}$. In this original form of the scenario (denoted in what follows by MSSMI ${ }_{\mathrm{o}}$ ), it is therefore natural to take a flat prior on $\phi_{0}$ such that $\phi_{0} / M_{\mathrm{P} 1} \in\left[2 \times 10^{-5}, 2 \times 10^{-4}\right]$.

This model can also be viewed as a phenomenological inflection point potential (denoted by $\operatorname{MSSMI}_{\mathrm{p}}$ ) where the value of $\phi_{0}$ is not fixed by high energy physics considerations. In that case, a Jeffreys prior on $\phi_{0}$ is appropriate and, here, we take $\log \left(\phi_{0} / M_{\mathrm{Pl}}\right) \in[-3,3]$.

\begin{tabular}{|l||c||c||c||c||c|}
\hline Name & Priors & $\ln \left(\mathcal{E} / \mathcal{E}_{\mathrm{HI}}\right)$ & $\mathcal{C}$ & $N$ & $\ln \left(\mathcal{L}_{\mathrm{max}} / \mathcal{E}_{\mathrm{HI}}\right)$ \\
\hline MSSMI $_{\mathrm{o}}$ & $\phi_{0} / M_{\mathrm{Pl}} \in\left[2 \times 10^{-5}, 2 \times 10^{-4}\right]$ & -10.27 & 0.76 & 3 & -7.40 \\
MSSMI $_{\mathrm{p}}$ & $\log \left(\phi_{0} / M_{\mathrm{Pl}}\right) \in[-3,3]$ & -3.28 & 4.61 & 3 & 1.72 \\
\hline
\end{tabular}




\section{A.19 Renormalisable Inflection Point Inflation (RIPI)}

This model is derived in the same context as MSSM inflation except that an additional term in the superpotential involving right handed neutrinos is considered. The amplitude of this new term is controlled by the dimensionless coupling constant $h \simeq 10^{-12}$. This gives rise to a new flat direction parametrised by the inflaton field $\phi$. This flat direction is lifted by the same mechanism discussed previously in the context of MSSM inflation and leads to the following potential [5]

$$
V(\phi)=M^{4}\left[\left(\frac{\phi}{\phi_{0}}\right)^{2}-\frac{4}{3}\left(\frac{\phi}{\phi_{0}}\right)^{3}+\frac{1}{2}\left(\frac{\phi}{\phi_{0}}\right)^{4}\right],
$$

where

$$
\phi_{0}=\sqrt{3} \frac{m_{\phi}}{h},
$$

$m_{\phi}$, as a soft breaking mass, being between $100 \mathrm{GeV}$ and $10 \mathrm{TeV}$. As a consequence, one has $\phi_{0} \sim 10^{14} \mathrm{GeV}$. For this version of the model (denoted as the "original version", RIPI o $^{\text {) a }}$ flat prior on $\phi_{0}$ represents the preferred choice, $\phi_{0} / M_{\mathrm{P} 1} \in\left[2 \times 10^{-5}, 2 \times 10^{-4}\right]$. As for MSSM inflation, however, one can also see this scenario as a phenomenological scenario where $\phi_{0}$ is not specified (denoted by RIPI $\mathrm{p}_{\mathrm{p}}$ in what follows). In this case, a Jeffreys prior on $\phi_{0}$ is natural and we take $\log \left(\phi_{0} / M_{\mathrm{Pl}}\right) \in[-3,3]$. Finally, the above potential can also arise in a supergravity framework with shift symmetry in the Kähler potential (denoted by RIPI $\mathrm{I}_{\text {sugra }}$ ) which allows for super-Planckian vev of the inflaton field $\phi$. For this reason, we also consider the prior $\phi_{0} / M_{\mathrm{P} 1} \in[10,50]$.

\begin{tabular}{|l||c||c||c||c||c|}
\hline Name & Priors & $\ln \left(\mathcal{E} / \mathcal{E}_{\mathrm{HI}}\right)$ & $\mathcal{C}$ & $N$ & $\ln \left(\mathcal{L}_{\max } / \mathcal{E}_{\mathrm{HI}}\right)$ \\
\hline $\mathrm{RIPI}_{\mathrm{O}}$ & $\phi_{0} / M_{\mathrm{Pl}} \in\left[2 \times 10^{-5}, 2 \times 10^{-4}\right]$ & -9.94 & 2.01 & 3 & -6.76 \\
RIPI $_{\mathrm{p}}$ & $\log \left(\phi_{0} / M_{\mathrm{Pl}}\right) \in[-3,3]$ & -2.31 & 3.60 & 3 & 2.19 \\
$\mathrm{RIPI}_{\text {sugra }}$ & $\phi_{0} / M_{\mathrm{Pl}} \sim[10,50]$ & -0.96 & 2.87 & 3 & 2.19 \\
\hline
\end{tabular}

\section{A.20 Arctan Inflation (AI)}

The potential of AI can be expressed as

$$
V(\phi)=M^{4}\left[1-\frac{2}{\pi} \arctan \left(\frac{\phi}{\mu}\right)\right],
$$

where $\mu$ is a free parameter. As shown in Ref. [5], inflation stops by slow-roll violation only if $\mu / M_{\mathrm{P} 1}<0.512378$. This model is purely phenomenological and, as a consequence, the scale $\mu$ is not fixed by any high energy physics considerations. As a consequence, its order of magnitude is a priori unspecified. Therefore, we choose a Jeffreys logarithmic prior on $\mu$, namely $\log \left(\mu / M_{\mathrm{Pl}}\right) \in[-3, \log (0.51 \cdots) \simeq-0.29]$.

\begin{tabular}{|l||c||c||c||c||c|}
\hline Name & Priors & $\ln \left(\mathcal{E} / \mathcal{E}_{\mathrm{HI}}\right)$ & $\mathcal{C}$ & $N$ & $\ln \left(\mathcal{L}_{\max } / \mathcal{E}_{\mathrm{HI}}\right)$ \\
\hline AI & $\log \left(\mu / M_{\mathrm{Pl}}\right) \in[-3,-0.29]$ & -0.20 & 2.18 & 3 & 2.29 \\
\hline
\end{tabular}

\section{A.21 Constant ns A Inflation (CNAI)}

The potential of CNAI is given by the following expression

$$
V(\phi)=M^{4}\left[3-\left(3+\alpha^{2}\right) \tanh ^{2}\left(\frac{\alpha}{\sqrt{2}} \frac{\phi}{M_{\mathrm{Pl}}}\right)\right],
$$


where $\alpha$ is a dimensionless free parameter. It was demonstrated in Ref. [5] that slow-roll inflation takes place provided $\alpha<\alpha_{\max } \simeq 0.66$. This model is phenomenological and is not based on high energy physics. It is in fact designed to produce an exact power law spectrum of density perturbations. As a consequence, the order of magnitude of $\alpha$ is not specified and one chooses to work with a Jeffreys prior $\log (\alpha) \in\left[-4, \log \left(\alpha_{\max }\right) \simeq-0.18\right]$.

\begin{tabular}{|l||c||c||c||c||c|}
\hline Name & Priors & $\ln \left(\mathcal{E} / \mathcal{E}_{\mathrm{HI}}\right)$ & $\mathcal{C}$ & $N$ & $\ln \left(\mathcal{L}_{\max } / \mathcal{E}_{\mathrm{HI}}\right)$ \\
\hline CNAI & $\log (\alpha) \in[-4,-0.18]$ & -1.96 & 2.22 & 3 & 0.79 \\
\hline
\end{tabular}

\section{A.22 Constant ns B Inflation (CNBI)}

This model is very similar to CNAI inflation. It is also a phenomenological scenario designed to produce a constant spectral index and also depends on one dimensionless parameter $\alpha$. The corresponding potential can be expressed as

$$
V(\phi)=M^{4}\left[\left(3-\alpha^{2}\right) \tan ^{2}\left(\frac{\alpha}{\sqrt{2}} \frac{\phi}{M_{\mathrm{Pl}}}\right)-3\right] .
$$

It was shown in Ref. [5] that slow-roll inflation takes place if $\alpha<\alpha_{\max } \simeq 0.2975$. If one CMB normalises the model, then one can express the mass scale $M$ in terms of $\alpha$. It follows that the requirement $M / M_{\mathrm{Pl}}<1$ implies $\alpha \lesssim 10^{-9}$. As a consequence, we should take a Jeffreys prior on $\alpha$, namely $\log (\alpha) \in\left[-9, \log \left(\alpha_{\max }\right) \simeq-0.527\right]$. In practice, however, when $\alpha>10^{-1.4}$, the likelihood is so small that it cannot be properly calculated. Moreover, when $\alpha<10^{-5}$, the value of the likelihood reaches a numerical stationary value and, therefore, it is not necessary to numerically calculate it beyond that point. As a consequence, we consider the following prior: $\log (\alpha) \in[-5,-1.4]$.

\begin{tabular}{|l||c||c||c||c||c|}
\hline Name & Priors & $\ln \left(\mathcal{E} / \mathcal{E}_{\mathrm{HI}}\right)$ & $\mathcal{C}$ & $N$ & $\ln \left(\mathcal{L}_{\max } / \mathcal{E}_{\mathrm{HI}}\right)$ \\
\hline CNBI & $\log (\alpha) \in[-5,-1.4]$ & -1.68 & 2.13 & 3 & 0.79 \\
\hline
\end{tabular}

\section{A.23 Open String Tachyonic Inflation (OSTI)}

In this model, the inflaton field is a tachyon field on a D3-brane. In principle, its kinetic term is non-minimal but when higher order terms are neglected, it becomes a standard slow-roll model with a potential given by the following expression

$$
V(\phi)=-M^{4}\left(\frac{\phi}{\phi_{0}}\right)^{2} \ln \left[\left(\frac{\phi}{\phi_{0}}\right)^{2}\right] .
$$

In the original version of the model, $\phi_{0}$ is set to the string scale $\phi_{0} \sim M_{\mathrm{s}}$. However, $\phi_{0}$ can also be viewed as a free sub-Planckian scale. In that case, a Jeffreys prior is appropriate, for instance $\log \left(\phi_{0} / M_{\mathrm{Pl}}\right) \in[0,4]$. However, when $\phi_{0} / M_{\mathrm{Pl}}<10$, the likelihood is so small that it cannot be numerically calculated in a reliable way. As a consequence, in what follows, we consider the prior $\log \left(\phi_{0} / M_{\mathrm{Pl}}\right) \in[1,4]$ only.

\begin{tabular}{|l||c||c||c||c||c|}
\hline Name & Priors & $\ln \left(\mathcal{E} / \mathcal{E}_{\mathrm{HI}}\right)$ & $\mathcal{C}$ & $N$ & $\ln \left(\mathcal{L}_{\max } / \mathcal{E}_{\mathrm{HI}}\right)$ \\
\hline OSTI & $\log \left(\phi_{0} / M_{\mathrm{Pl}}\right) \in[1,4]$ & -1.87 & 4.27 & 3 & 2.14 \\
\hline
\end{tabular}




\section{A.24 Witten-O'Raifeartaigh Inflation (WRI)}

The potential of WRI inflation can be expressed as

$$
V(\phi)=M^{4} \ln \left(\frac{\phi}{\phi_{0}}\right)^{2}
$$

When the high energy justifications of the scenario are considered, the condition $\phi_{0}=M_{\mathrm{P} 1}$ holds. In what follows, we call this version of the model the "original WRI" and we denote it as $\mathrm{WRI}_{\mathrm{O}}$. If this condition is relaxed (the corresponding version of the model is then denoted by $\mathrm{WRI}_{\mathrm{g}}$ ) and if the model is now viewed as a more phenomenological one, then the order of magnitude and value of $\phi_{0}$ are unspecified and a Jeffreys prior is appropriate. We choose $\log \left(\phi_{0} / M_{\mathrm{P} 1}\right) \in[-3,3]$. These considerations are summarised in the following table:

\begin{tabular}{|c||c||c||c||c||c|}
\hline Name & Priors & $\ln \left(\mathcal{E} / \mathcal{E}_{\mathrm{HI}}\right)$ & $\mathcal{C}$ & $N$ & $\ln \left(\mathcal{L}_{\mathrm{max}} / \mathcal{E}_{\mathrm{HI}}\right)$ \\
\hline $\mathrm{WRI}_{\mathrm{O}}$ & $\phi_{0}=M_{\mathrm{Pl}}$ & -1.09 & 2.05 & 2 & 1.29 \\
$\mathrm{WRI}_{\mathrm{g}}$ & $\log \left(\phi_{0} / M_{\mathrm{Pl}}\right) \in[-3,3]$ & -1.20 & 2.97 & 3 & 1.89 \\
\hline
\end{tabular}

\section{A.25 Small Field Inflation (SFI)}

Small field inflation is characterised by the following potential

$$
V(\phi)=M^{4}\left[1-\left(\frac{\phi}{\mu}\right)^{p}\right]
$$

which depends on two parameters, the dimensionless index $p$ and the mass scale $\mu$. In most of high energy physics implementations, only the case $\mu<M_{\mathrm{Pl}}$ is sensible. It is, however, always possible to take a more phenomenological point of view and also consider the case $\mu>M_{\mathrm{Pl}}$. In what follows, for this reason, we will discuss a "small" version of the scenario for which $\log \left(\mu / M_{\mathrm{Pl}}\right) \in[-1,0]$ and a "large" version for which $\log \left(\mu / M_{\mathrm{Pl}}\right) \in[0,2]$. Two remarks are in order at this point. Firstly, a Jeffreys prior is chosen on $\mu$ because, a priori, its order of magnitude is unspecified. Secondly, in the small version of the model, we only consider $\log \left(\mu / M_{\mathrm{Pl}}\right) \in[-1,0]$ (and not, for instance, $\log \left(\mu / M_{\mathrm{Pl}}\right) \in[-2,0]$ ) because, when $\mu / M_{\mathrm{Pl}}<0.1$, the likelihood is so small that it cannot be properly numerically calculated.

The index $p$ is an $\mathcal{O}(1)$ parameter that can also take specific integer values. We will treat the case where there is a flat prior on $p$, namely $p \in[2,10]$, but also the case where $p$ has specific values, $p=1, p=2, p=3$ and $p=4$. Let us also notice that for $p=1$ and $p=2$, the small version of the SFI inflation does not exist because slow-roll is violated in that case (for instance, for $p=2$, one has $\epsilon_{2}>4$ ).

Our priors are summarised in the following table: 


\begin{tabular}{|l||c||c||c||c||c|}
\hline Name & Priors & $\ln \left(\mathcal{E} / \mathcal{E}_{\mathrm{HI}}\right)$ & $\mathcal{C}$ & $N$ & $\ln \left(\mathcal{L}_{\mathrm{max}} / \mathcal{E}_{\mathrm{HI}}\right)$ \\
\hline $\mathrm{SFI}$ & $\begin{array}{c}p \in[2,10] \\
\log \left(\mu / M_{\mathrm{Pl}}\right) \in[-1,2]\end{array}$ & -0.31 & 1.88 & 4 & 2.29 \\
\hline $\mathrm{SFI}_{\mathrm{S}}$ & $\begin{array}{c}p \in[2,10] \\
\log \left(\mu / M_{\mathrm{Pl}}\right) \in[-1,0]\end{array}$ & -0.40 & 1.54 & 4 & 2.27 \\
\hline $\mathrm{SFI}_{1}$ & $\begin{array}{c}p \in[2,10] \\
\log \left(\mu / M_{\mathrm{Pl}}\right) \in[0,2]\end{array}$ & -0.30 & 2.01 & 4 & 2.29 \\
\hline $\mathrm{SFI}_{1}$ & $\begin{array}{c}p=1 \\
\log \left(\mu / M_{\mathrm{Pl}}\right) \in[-1,2]\end{array}$ & -1.53 & 1.94 & 3 & 0.79 \\
\hline $\mathrm{SFI}_{2}$ & $\begin{array}{c}p=2 \\
\log \left(\mu / M_{\mathrm{Pl}}\right) \in[-1,2]\end{array}$ & -1.90 & 3.07 & 3 & 2.19 \\
\hline $\mathrm{SFI}_{21}$ & $\begin{array}{c}p=2 \\
\log \left(\mu / M_{\mathrm{Pl}}\right) \in[0,2]\end{array}$ & -1.47 & 3.07 & 3 & 2.19 \\
\hline $\mathrm{SFI}_{3}$ & $\begin{array}{c}p=3 \\
\log \left(\mu / M_{\mathrm{Pl}}\right) \in[-1,2]\end{array}$ & -1.23 & 2.74 & 3 & 2.26 \\
\hline $\mathrm{SFI}_{3 \mathrm{~s}}$ & $\begin{array}{c}p=3 \\
\log \left(\mu / M_{\mathrm{Pl}}\right) \in[-1,0]\end{array}$ & -3.88 & 3.13 & 3 & 0.67 \\
\hline $\mathrm{SFI}_{31}$ & $\begin{array}{c}p=3 \\
\log \left(\mu / M_{\mathrm{Pl}}\right) \in[0,2]\end{array}$ & -0.87 & 2.65 & 3 & 2.26 \\
\hline $\mathrm{SFI}_{4}$ & $\begin{array}{c}p=4 \\
\log \left(\mu / M_{\mathrm{Pl}}\right) \in[-1,2]\end{array}$ & -0.53 & 2.12 & 3 & 2.29 \\
\hline $\mathrm{SFI}_{4 \mathrm{~s}}$ & $\begin{array}{c}p=4 \\
\log \left(\mu / M_{\mathrm{Pl}}\right) \in[-1,0]\end{array}$ & -0.79 & 1.95 & 3 & 2.26 \\
\hline $\mathrm{SFI}_{41}$ & $\begin{array}{c}p=4 \\
\log \left(\mu / M_{\mathrm{Pl}}\right) \in[0,2]\end{array}$ & -0.41 & 2.14 & 3 & 2.29 \\
\hline
\end{tabular}

\section{A.26 Intermediate Inflation (II)}

Intermediate Inflation is a phenomenological model that can be defined by demanding an equation of state during inflation of the form

$$
\rho+p=\gamma \rho^{\lambda},
$$

where $\gamma>0$ and $\lambda>1$ are dimensionless parameters, $\rho$ and $p$ being the energy density and pressure stored in the inflaton field, respectively. This assumption is in fact equivalent to having a scale factor given by $a(t) \propto \exp \left(A t^{f}\right)$ where

$$
f=\frac{2(1-\lambda)}{1-2 \lambda} .
$$

Given that $\lambda>1$, it follows that $0<f<1$. Finally, it is also equivalent to postulate the following potential

$$
V(\phi)=M^{4}\left[\left(\frac{\phi}{M_{\mathrm{Pl}}}\right)^{-\beta}-\frac{\beta^{2}}{6}\left(\frac{\phi}{M_{\mathrm{Pl}}}\right)^{-\beta-2}\right],
$$

with

$$
\beta=4\left(\frac{1}{f}-1\right) .
$$

In this scenario, inflation cannot stop by violation of the slow-roll conditions and, hence, one needs to postulate an extra mechanism such as tachyonic instability. This implies that the 
scenario depends on another parameter, $\phi_{\text {end }}$, the vev at which inflation ends. Intermediate inflation is therefore a two parameters models, $\phi_{\text {end }}$ and $\lambda$ (or $f$ or $\beta$ ).

Given the above considerations, one can choose to take a flat prior on $\beta \in[0,10]$ (in the following, we denote the corresponding version of the scenario by $\mathrm{II}_{\beta}$ ). It makes also sense to work with a flat prior on $f \in[0,1]$ (this version of the model is denoted $\mathrm{II}_{f}$ ). In fact, in order to avoid an infinite value of $\beta$, we will consider the following prior $f \in[0.1,1]$. Finally, we also investigate a Jeffreys prior on $\lambda$ (this version is denoted by $\mathrm{II}_{\lambda}$ ), namely $\log (\lambda) \in[0.1,4]$, the lower bound $\log (\lambda)>0.1$ being chosen to have finite values of $\beta$.

The prior on $\phi_{\text {end }}$ also needs to be discussed. It was shown in Ref. [5] that the parameter $x_{\text {end }}=\phi_{\text {end }} / M_{\mathrm{P} 1}$ must be larger than some value $x_{\text {end }}^{\text {min }}$ in order to have a sufficient number of $e$-folds during inflation. The parameter $x_{\text {end }}$ is only known numerically and has been calculated in Ref. [5]. Moreover, the order of magnitude of $x_{\text {end }}$ is not known and, therefore, this suggests a Jeffreys prior. As a consequence, we take $\log \left(x_{\text {end }}\right) \in\left[\log \left(x_{\text {end }}^{\min }\right), 4\right]$. Everything is summarised in the following table.

\begin{tabular}{|l||c||c||c||c||c|}
\hline Name & Priors & $\ln \left(\mathcal{E} / \mathcal{E}_{\mathrm{HI}}\right)$ & $\mathcal{C}$ & $N$ & $\ln \left(\mathcal{L}_{\max } / \mathcal{E}_{\mathrm{HI}}\right)$ \\
\hline $\mathrm{II}_{\beta}$ & $\begin{array}{c}\beta \in[0,10] \\
\log \left(x_{\text {end }}\right) \in\left[\log \left(x_{\text {end }}^{\text {min }}\right), 4\right]\end{array}$ & -7.61 & 7.39 & 4 & -3.66 \\
\hline $\mathrm{II}_{f}$ & $\begin{array}{c}f=1 /(1+\beta / 4) \in[0.1,1] \\
\log \left(x_{\text {end }}\right) \in\left[\log \left(x_{\text {end }}^{\text {min }}\right), 4\right]\end{array}$ & -7.56 & 8.22 & 4 & -3.02 \\
\hline $\mathrm{II}_{\lambda}$ & $\begin{array}{c}\log (\lambda)=\log (1+2 / \beta) \in[0.1,4] \\
\log \left(x_{\text {end }}\right) \in\left[\log \left(x_{\text {end }}^{\text {min }}\right), 4\right]\end{array}$ & -7.79 & 7.79 & 4 & -3.89 \\
\hline
\end{tabular}

\section{A.27 Kähler Moduli Inflation II (KMIII)}

Kähler Moduli Inflation III is a stringy inspired scenario the potential of which can be written as

$$
V(\phi)=M^{4}\left[1-\alpha\left(\frac{\phi}{M_{\mathrm{Pl}}}\right)^{4 / 3} e^{-\beta\left(\phi / M_{\mathrm{Pl}}\right)^{4 / 3}}\right] .
$$

In this model, the inflaton field is a modulus field. The potential depends on two parameters, $\alpha$ and $\beta$. As reviewed in Ref. [5], the order of magnitude of the parameter $\beta$ is in fact controlled by the compactification volume $\mathcal{V}$. More precisely, one can show that $\alpha=\mathcal{O}\left(\mathcal{V}_{\mathrm{s}}^{5 / 3}\right)$ and $\beta=\mathcal{O}\left(\mathcal{V}_{\mathrm{s}}^{2 / 3}\right)$ where $\mathcal{V}_{\mathrm{s}}$ is a dimensionless volume defined by $\mathcal{V}_{\mathrm{s}}=\mathcal{V} / \ell_{\mathrm{s}}^{6}, \ell_{\mathrm{s}}$ being the string length. Since typical values are usually chosen such that $\mathcal{V}_{\mathrm{s}} \sim 10^{6}$ and since the order of magnitude of $\mathcal{V}_{\mathrm{s}}$ is not precisely specified, we take a logarithmic prior on $\mathcal{V}_{\mathrm{s}}$, namely $\log (\mathcal{V}) \in[5,7]$.

On the other hand, the ratio $\alpha /(\beta \mathcal{V})$ is a $\mathcal{O}(1)$ quantity, thanks to the scaling mentioned above. As a consequence, we choose a flat prior $\alpha /(\beta \mathcal{V}) \in[0.2,5]$. In practice, once the number $\mathcal{V}_{\mathrm{s}}$ is fixed, one calculate $\beta$ by means of $\beta=\mathcal{V}_{\mathrm{s}}^{2 / 3}$. Then, the ratio $\alpha /(\beta \mathcal{V})$ is chosen and one deduces the value of $\alpha$.

\begin{tabular}{|c||c||c||c||c||c|}
\hline Name & Priors & $\ln \left(\mathcal{E} / \mathcal{E}_{\mathrm{HI}}\right)$ & $\mathcal{C}$ & $N$ & $\ln \left(\mathcal{L}_{\max } / \mathcal{E}_{\mathrm{HI}}\right)$ \\
\hline KMIII & $\begin{array}{c}\log (\mathcal{V}) \in[5,7] \\
\alpha /(\beta \mathcal{V}) \in[0.2,5]\end{array}$ & 0.07 & 1.66 & 3 & 2.22 \\
\hline
\end{tabular}




\section{A.28 Logamediate inflation (LMI)}

This model is a phenomenological model designed such that the scale factor during inflation behaves as

$$
a(t)=a_{0} \exp \left[A\left(\ln \frac{t}{t_{0}}\right)^{\lambda}\right],
$$

where $A>0$ and $\lambda>1$ are two dimensionless parameters and $t_{0}$ is a third parameter the dimension of which is time. From this expression of the scale factor, one can infer the shape of the potential. Straightforward calculations [5] lead to

$$
V(\phi)=M^{4}\left(\frac{\phi}{M_{\mathrm{Pl}}}\right)^{4(1-\gamma)} \exp \left[-\beta\left(\frac{\phi}{M_{\mathrm{Pl}}}\right)^{\gamma}\right]
$$

where the parameters $\gamma$ and $\beta$ can be expressed as

$$
\gamma=\frac{2}{\lambda+1}, \quad \beta=2\left(\frac{\lambda+1}{2 \sqrt{2 A \lambda}}\right)^{2 /(\lambda+1)} .
$$

These relations, together with the conditions on $A$ and $\lambda$, imply $0<\gamma \leq 1$ and $\beta>0$. The potential (A.43) has a maximum located at

$$
x_{\max } \equiv \frac{\phi_{\max }}{M_{\mathrm{Pl}}}=\left[\frac{4(1-\gamma)}{\beta \gamma}\right]^{1 / \gamma}
$$

This gives rise to two different versions of the model [5]: either inflation proceeds on the left side of its maximum and the field vev decreases during inflation (we call this version LMI1 in the following) or it proceeds on the right side of its maximum and the field vev increases during inflation (this version is denoted LMI2). In the case of LMI1, inflation stops by slow-roll violation. The case of LMI2 is more complicated but, in brief, one needs an extra mechanism to end inflation and this introduces a new parameter in the model, $x_{\text {end }}$, see Ref. [5] for more details. LMI2 is therefore a three parameter model.

Regarding the priors, we essentially have two choices: either we specify them on the parameters characterising the potential or we specify them on the parameters controlling the behaviour of the scale factor. In the following, we consider both cases.

Let us start with the case where we choose priors on the parameters of the potential. In the following, we denote the two corresponding versions of the scenario by LMI $1_{p}$ and LMI $2_{\mathrm{p}}$. For LMI1 $1_{\mathrm{p}}$, it is natural to take a flat prior on $\gamma$, namely $\gamma \in[0,1]$. In fact, $\gamma=0$ is numerically pathological and, therefore, in practice, we consider $\gamma \in[0.1,1]$. For the parameter $\beta$, one takes a flat prior $\beta \in\left[0.01, \beta_{\max }(\gamma)\right]$, where

$$
\beta_{\max }(\gamma)=2^{2-3 \gamma / 2}(0.1)^{\gamma / 2} \frac{(1-\gamma)^{1-\gamma / 2}}{\gamma^{1+\gamma / 2}}
$$

As discussed in Ref. [5], the condition $\beta<\beta_{\max }(\gamma)$ is mandatory in order for the slow-roll conditions to be valid.

Let us now turn to LMI2 $2_{\mathrm{p}}$. For this case, we also consider a flat prior on $\gamma, \gamma \in[0.1,0.99]$. For this model, there is no condition on $\beta$ in order to satisfy the slow-roll and, therefore, one takes a flat prior on this parameter, namely $\beta \in[0.01,10]$. Finally, the order of magnitude of $x_{\text {end }}$ is not specified and this suggests a Jeffreys prior. Notice also that one must have 
$x_{\text {end }}>x_{\text {end }}^{\min }\left(\gamma, \beta, \Delta N_{\min }\right)$ in order to have at least $\Delta N_{\min }$ e-folds during inflation (typically $\Delta N_{\text {min }} \simeq 50$ ). Combining these two pieces of information leads us to the following prior $\log \left(x_{\text {end }}\right) \in\left[\log \left(x_{\text {end }}^{\min }\right), \log \left(100 x_{\text {end }}^{\min }\right)\right]$.

Let us now treat the case where the priors are chosen from considerations based on the form of the scale factor (A.42). We denote these versions $\mathrm{LMI}_{\mathrm{o}}$ and $\mathrm{LMI} 2_{\mathrm{o}}$. This means that we first choose $A$ and $\lambda$ and then infer $\gamma$ and $\beta$ from Eqs. (A.44). For the LMI1 o model, since $\lambda$ is a $\mathcal{O}(1)$ parameter, one takes a flat prior on this parameter, namely $\lambda \in[1,6]$. For the parameter $A$, one needs to take into account the fact that there is a maximum value of $\beta$, see the above discussion. In fact, it is possible to invert Eqs. (A.44) and to express $A$ in terms of $\beta$ and $\gamma$. One finds

$$
A=\left(\frac{2}{\beta}\right)^{2 / \gamma}\left(\frac{2}{\gamma}\right)^{2} \frac{1}{8(2 / \gamma-1)}
$$

Given that $2 / \gamma>1$, the presence of a $\beta_{\max }$ implies a $A_{\min }$ which can be expressed as

$$
A_{\min }=\left(\frac{2}{\beta_{\max }}\right)^{2 / \gamma}\left(\frac{2}{\gamma}\right)^{2} \frac{1}{8(2 / \gamma-1)} .
$$

In addition, since the order of magnitude of $A$ is a priori not fixed, one chooses to work with a Jeffreys prior. We therefore take $\log (A) \in\left[A_{\min }(\lambda), 2\right]$.

Let us finally examine the LMI2 $2_{\mathrm{o}}$ version. We take the same prior on $\lambda$ and $x_{\text {end }}$ as before. Since there is no maximum value of $\beta$ anymore, there is no minimal value of $A$. As a consequence, we work with the following prior on $A$ : $\log (A) \in[-2,2]$.

\begin{tabular}{|c|c|c|c|c|c|}
\hline Name & Priors & $\ln \left(\mathcal{E} / \mathcal{E}_{\mathrm{HI}}\right)$ & $\mathcal{C}$ & $N$ & $\ln \left(\mathcal{L}_{\max } / \mathcal{E}_{\mathrm{HI}}\right)$ \\
\hline $\mathrm{LMI}_{\mathrm{p}}$ & $\begin{array}{c}\gamma \in[0.1,1] \\
\beta \in\left[0.01, \beta_{\max }(\gamma)\right]\end{array}$ & -1.36 & 3.06 & 4 & 2.29 \\
\hline $\mathrm{LMI}_{\mathrm{o}}$ & $\begin{array}{c}\lambda \in[1,6] \\
\log (A) \in\left[A_{\min }(\lambda), 2\right]\end{array}$ & -0.99 & 2.83 & 4 & 2.24 \\
\hline $\mathrm{LMI}_{2}$ & $\begin{array}{c}\gamma \in[0.1,0.99] \\
\beta \in[0.01,10] \\
\log \left(x_{\text {end }}\right) \in\left[\log \left(x_{\text {end }}^{\min }\right), \log \left(100 x_{\text {end }}^{\min }\right)\right]\end{array}$ & -4.35 & 3.74 & 5 & 2.29 \\
\hline $\mathrm{LMI} 2_{\mathrm{o}}$ & $\begin{array}{c}\lambda \in[1.1,6] \\
\log (A) \in[-2,2] \\
\log \left(x_{\text {end }}\right) \in\left[\log \left(x_{\text {end }}^{\min }\right), \log \left(100 x_{\text {end }}^{\min }\right)\right]\end{array}$ & -3.93 & 3.24 & 5 & 2.29 \\
\hline
\end{tabular}

All the above considerations are summarised in the following table:

\section{A.29 Twisted inflation (TWI)}

The potential of Twisted Inflation (TWI) is given by the following expression

$$
V(\phi)=M^{4}\left[1-A\left(\frac{\phi}{\phi_{0}}\right)^{2} e^{-\phi / \phi_{0}}\right],
$$

where the two parameters $M$ and $\phi_{0}$ can be expressed as

$$
M^{4}=\frac{8 \mathcal{N}}{A \pi^{2}(2 \pi R)^{4}}, \quad \frac{\phi_{0}}{M_{\mathrm{P} 1}}=\frac{1}{2 \pi R M_{\mathrm{Pl}}},
$$


the constant $A$ being defined by $A=32 /[93 \zeta(5)] \simeq 0.33$. This model is based on higher dimensional supersymmetric gauge theories, more precisely $\mathrm{U}(\mathcal{N})$ Yang-Mills theory, and $R$ represents the radius of compactification. The above potential is valid provided $R M_{\mathrm{Pl}} \gg 1$, that is to say $\phi_{0} / M_{\mathrm{Pl}} \ll 1$. In fact, the model makes sense if $\phi \ll M_{\mathrm{Pl}}$ for any vev and not only $\phi_{0}$, see Ref. [5] for more detail. Inflation cannot stop by violation of the slow-roll conditions and, as a consequence, one needs to introduce another mechanism which is characterised by a new parameter, $\phi_{\text {end }}$. TWI inflation is therefore a two parameter model.

Let us now discuss the priors. We have just seen that $\phi_{0}$ must be sub-Planckian. Since its order of magnitude is a priori unknown, it seems natural to take a Jeffreys prior, namely $\log \left(\phi_{0} / M_{\mathrm{Pl}}\right) \in[-4,-1]$. Concerning the vev at which inflation ends, we know that $\phi_{\text {end }} / \phi_{0}>2$ because the minimum of the potential is located at $\phi / \phi_{0}=2$. Otherwise, as already discussed, the only other constraint is $\phi_{\text {end }} \ll M_{\mathrm{Pl}}$. However, in practice, for values of $\phi_{\text {end }}$ approaching the Planck mass, the potential is so flat that this regime is already strongly disfavoured (because $n_{\mathrm{S}} \simeq 1$ ). Therefore, it is better to choose an upper bound for $\log \left(\phi_{\mathrm{end}} / \phi_{0}\right)$ supplemented with the hard prior $\phi_{\mathrm{end}}<M_{\mathrm{Pl}}$. Then, one can study if the evidence is changed if we modify the upper bound. Since the order of magnitude of this parameter is a priori not specified, we must also take a Jeffreys prior on $\phi_{0}$. To summarise, we consider the two following priors $\log \left(\phi_{\text {end }} / \phi_{0}\right) \in[\log (2), \log (20)]$ and $\log \left(\phi_{\text {end }} / \phi_{0}\right) \in$ $[\log (2), \log (40)]$ and check that, indeed, the final result is not sensitive to the upper bound. In the following, we denote these priors by $\mathrm{TWI}_{\phi_{0}}$ and $\mathrm{TWI}_{\phi_{0}}^{\mathrm{r}}$.

At the fundamental level, Twisted Inflation is in fact characterised by $\mathcal{N}$ and not by $\phi_{0}$. If we CMB normalise the model, one can express the latter in terms of the former, namely $\phi_{0} / M_{\mathrm{P} 1} \simeq 10^{-5} / \sqrt{\mathcal{N}}$. In this version of the model, denoted by TWI and TWI ${ }^{\mathrm{r}}$ in what follows, the prior choices are now fixed on $\mathcal{N}$ (and the value of $\phi_{0}$ is calculated using the above equation). Since $\mathcal{N}$ is a priori a number of order one, it makes sense to take a flat prior and we choose $\mathcal{N} \in[1,100]$. Concerning $\phi_{\text {end }}$, we just take the same priors as before.

\begin{tabular}{|c||c||c||c||c||c|}
\hline Name & Priors & $\ln \left(\mathcal{E} / \mathcal{E}_{\mathrm{HI}}\right)$ & $\mathcal{C}$ & $N$ & $\ln \left(\mathcal{L}_{\max } / \mathcal{E}_{\mathrm{HI}}\right)$ \\
\hline \multirow{2}{*}{$\mathrm{TWI}_{\phi_{0}}$} & $\begin{array}{c}\log \left(\phi_{0} / M_{\mathrm{Pl}}\right) \in[-4,-1] \\
\log \left(\phi_{\text {end }} / \phi_{0}\right) \in[\log (2), \log (20)] \\
\phi_{\text {end }}<M_{\mathrm{P} 1}\end{array}$ & -0.73 & 1.64 & 4 & 2.27 \\
\hline \multirow{2}{*}{$\mathrm{TWI}_{\phi_{0}}^{\mathrm{r}}$} & $\begin{array}{c}\log \left(\phi_{0} / M_{\mathrm{Pl}}\right) \in[-4,-1] \\
\log \left(\phi_{\text {end }} / \phi_{0}\right) \in[\log (2), \log (40)] \\
\phi_{\text {end }}<M_{\mathrm{P} 1}\end{array}$ & -0.83 & 1.66 & 4 & 2.27 \\
\hline \multirow{2}{*}{$\mathrm{TWI}$} & $\begin{array}{c}\mathcal{N}=10^{-10}\left(\phi_{0} / M_{\mathrm{Pl}}\right)^{-2} \in[1,100] \\
\log \left(\phi_{\text {end }} / \phi_{0}\right) \in[\log (2), \log (20)] \\
\phi_{\text {end }}<M_{\mathrm{Pl}}\end{array}$ & -2.74 & 1.50 & 4 & 2.27 \\
\hline \multirow{2}{*}{$\mathrm{TWI}$} & $\begin{array}{c}\mathcal{N}=10^{-10}\left(\phi_{0} / M_{\mathrm{Pl}}\right)^{-2} \in[1,100] \\
\log \left(\phi_{\text {end }} / \phi_{0}\right) \in[\log (2), \log (40)] \\
\phi_{\text {end }}<M_{\mathrm{Pl}}\end{array}$ & -1.55 & 1.55 & 4 & 2.27 \\
\hline
\end{tabular}

\section{A.30 Generalised MSSM Inflation (GMSSMI)}

This model is a generalisation of MSSMI studied in section A.18. The potential can be expressed as [5]

$$
V(\phi)=M^{4}\left[\left(\frac{\phi}{\phi_{0}}\right)^{2}-\frac{2}{3} \alpha\left(\frac{\phi}{\phi_{0}}\right)^{6}+\frac{\alpha}{5}\left(\frac{\phi}{\phi_{0}}\right)^{10}\right] .
$$


This is a two-parameters model, $\phi_{0}$ and $\alpha$, and the potential of MSSMI is recovered for $\alpha=1$. As already discussed in section A.18 and in Ref. [5], the typical value for the vev $\phi_{0}$ is $\phi_{0} \simeq 10^{14} \mathrm{GeV}$. The model can also be viewed as a phenomenological one, that is to say as a representative of the class of the so-called inflection point inflationary scenario.

Let us now discuss the priors. Viewed as a phenomenological model (denoted by GMSSMI $_{\mathrm{p}}$ in what follows), the model is such that the scale of $\phi_{0}$ is unspecified and, therefore, a Jeffreys prior is appropriate. We choose to work with $\log \left(\phi_{0} / M_{\mathrm{Pl}}\right) \in[-5,5]$. On the other hand, the parameter $\alpha$ is of order one and, as a consequence, we take a flat prior: $\alpha \in[0.9,1.1]$. Finally, a hard prior has been implemented to reject all non slow-roll cases (defined to have $\left|\epsilon_{2}\right|>0.2$ ).

If we now want to calculate the evidence of the model motivated by particle physics, we must include in the analysis the fact that the vev $\phi_{0}$ is around $10^{14} \mathrm{GeV}$. For this reason, we choose a flat prior such that $\phi_{0} / M_{\mathrm{Pl}} \in\left[2 \times 10^{-5}, 2 \times 10^{-4}\right]$. One also knows that, if $\alpha$ is not precisely tuned around $\alpha=1$, then the model can not support slow-roll inflation and is, therefore, ruled out. Moreover, requiring at least $\Delta N \simeq 60 e$-fold during inflation leads to the constraint

$$
|\alpha-1|<\frac{\phi_{0}^{4}}{M_{\mathrm{Pl}}^{4}} \frac{\pi^{2}}{900 \Delta N^{2}},
$$

see Ref. [5]. This formula tells us that, if $|\alpha-1| \gtrsim 10^{-20}$, then the model is ruled out. This illustrates the extreme fine-tuning needed for this model to be compatible with the Planck data. When $\alpha>1$, we implement this fine-tuning through two different choices of priors satisfying the above condition, namely $\log (1-\alpha) \in[-28,-23]$ and $\log (1-\alpha) \in[-28,-20]$, corresponding to the $\mathrm{GMSSMI}_{\mathrm{omA}}$ and $\mathrm{GMSSMI}_{\mathrm{omB}}$ versions of the model, GMSSMI $\mathrm{OmB}_{\mathrm{om}}$ being on the validity threshold. If $\alpha>1$, we define two other models denoted GMSSMI ${ }_{\mathrm{opA}}$ and GMSSMI $_{\mathrm{opB}}$ such that $\log (\alpha-1) \in[-28,-23]$ and $\log (\alpha-1) \in[-28,-21.75]$. Our choices for the priors are summarised in the following table:

\begin{tabular}{|c|c|c|c|c|c|}
\hline Name & Priors & $\ln \left(\mathcal{E} / \mathcal{E}_{\mathrm{HI}}\right)$ & $\mathcal{C}$ & $N$ & $\ln \left(\mathcal{L}_{\max } / \mathcal{E}_{\mathrm{HI}}\right)$ \\
\hline GMSSMI $_{\mathrm{p}}$ & $\begin{array}{c}\log \left(\phi_{0} / M_{\mathrm{Pl}}\right) \in[-5,5] \\
\alpha \in[0.9,1.1]\end{array}$ & -2.54 & 4.96 & 4 & 1.77 \\
\hline $\mathrm{GMSSMI}_{\mathrm{opA}}$ & $\begin{array}{c}\phi_{0} / M_{\mathrm{Pl}} \in\left[2 \times 10^{-5}, 2 \times 10^{-4}\right] \\
\log (\alpha-1) \in[-28,-23] \\
\ln R \in[-46,0], \Delta N>60\end{array}$ & -10.30 & 0.76 & 4 & -7.40 \\
\hline $\mathrm{GMSSMI}_{\mathrm{opB}}$ & $\begin{array}{c}\phi_{0} / M_{\mathrm{Pl}} \in\left[2 \times 10^{-5}, 2 \times 10^{-4}\right] \\
\log (\alpha-1) \in[-28,-21.75] \\
\ln R \in[-46,0], \Delta N>60\end{array}$ & -10.41 & 0.76 & 4 & -7.40 \\
\hline GMSSMI $_{\mathrm{omA}}$ & $\begin{array}{c}\phi_{0} / M_{\mathrm{PI}} \in\left[2 \times 10^{-5}, 2 \times 10^{-4}\right] \\
\log (1-\alpha) \in[-28,-23] \\
\ln R \in[-46,0], \Delta N>60\end{array}$ & -7.85 & 5.39 & 4 & 2.23 \\
\hline GMSSMI $_{\mathrm{omB}}$ & $\begin{array}{c}\phi_{0} / M_{\mathrm{PI}} \in\left[2 \times 10^{-5}, 2 \times 10^{-4}\right] \\
\log (1-\alpha) \in[-28,-20] \\
\ln R \in[-46,0], \Delta N>60\end{array}$ & -5.25 & 2.87 & 4 & 2.27 \\
\hline
\end{tabular}

One may also wonder how the evidence would be changed if one penetrates the regime where Eq. (A.52) is not satisfied (and where the slow-roll approximation is not satisfied). In that case, since all non slow-roll models are incompatible with the Planck data, the evidence should only be rescaled by the ratio of the prior volumes. Therefore, in the following, we 


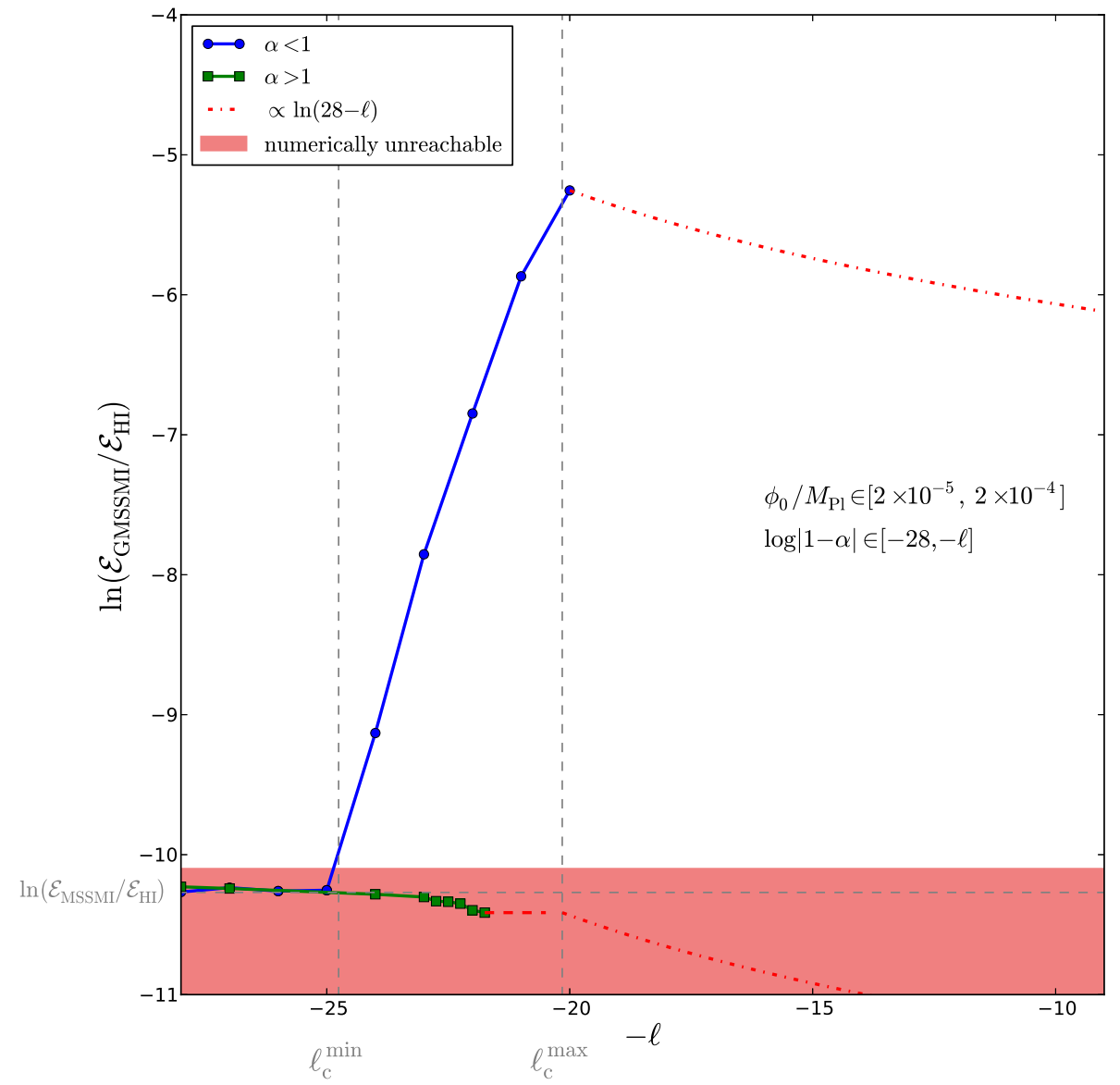

Figure 5. Evolution of the GMSSMI Bayes factor versus the upper bound $-\ell$ of the prior range on $\alpha$ for $\alpha>1$ and $\alpha<1$. The green squares and blue circles represent numerical values of the evidence. The dotted red curves represent the analytical laws giving the behaviour of the Bayes factor versus $-\ell$ for $-\ell \gtrsim \ell_{\mathrm{c}}^{\max }$ according to Eqs. (A.53) and (A.54). These equations predict how the Bayes factor behaves with $-\ell$ and, therefore, can be used to extrapolate in regimes where $\alpha$ becomes of order one.

study the more general situation where $\log |\alpha-1| \in[-28,-\ell]$, where $\ell$ is the variable with respect to which we want to study the behaviour of the Bayesian evidence. In the prior plane $\left[\phi_{0} / M_{\mathrm{P} 1}, \log |1-\alpha|\right]$, Eq. (A.52) defines a line above which the likelihood vanishes (since, in that case and as already mentioned, the model becomes incompatible with the data). This curve approximately goes from $\left(2 \times 10^{-5},-24 \equiv \ell_{\mathrm{c}}^{\min }\right)$ to $\left(2 \times 10^{-4},-20 \equiv \ell_{\mathrm{c}}^{\max }\right)$ and, therefore, defines three different regions according to whether $-\ell<\ell_{\mathrm{c}}^{\min },-\ell \in\left[\ell_{\mathrm{c}}^{\min }, \ell_{\mathrm{c}}^{\max }\right]$ or $-\ell>\ell_{\mathrm{c}}^{\max }$.

Let us first assume that $\alpha>1$ and $\log (\alpha-1) \in[-28,-\ell]$. If $-\ell \lesssim-24$, then $\alpha-1$ is so small that one expects the model to be equivalent to MSSMI. If $\ell \in\left[\ell_{\mathrm{c}}^{\min }, \ell_{\mathrm{c}}^{\max }\right]$ (denoted the "transition region" in what follows), then only numerical calculations can track the behaviour of the evidence. Notice that $\mathrm{GMSSMI}_{\mathrm{opA}}$ and $\mathrm{GMSSMI}_{\mathrm{opB}}$ belongs to this region. Finally, for $-\ell>\ell_{\mathrm{c}}^{\max }$, one expects the evidence to scale with the ratio of the prior volumes. These expectations are confirmed in Fig. 5 (solid green line). However, for numerical reasons, we 
are in fact unable to follow the evidence beyond the point $-\ell \simeq-21.75$ (GMSSMI $_{\text {opB }}$ model) which is still in the transition region. One can nevertheless assume that the evidence does not change much between that point and the edge of the transition region (hence the small horizontal dashed red segment inside the transition region in Fig. 5). In that case, in the regime $-\ell>\ell_{\mathrm{c}}^{\max }$, one can write

$$
\ln \left[\frac{\mathcal{E}_{\log (\alpha-1) \in[-28,-\ell]}}{\mathcal{E}_{\mathrm{HI}}}\right] \simeq \ln \left(\frac{\mathcal{E}_{\mathrm{GMSSMI}}{ }_{\mathcal{E}_{\mathrm{HP}}}}{\mathcal{H I}}\right)+\ln \left(28-\ell_{\mathrm{c}}^{\max }\right)-\ln (28-\ell) .
$$

This rough approximation can be considered as reasonable because it gives an upper bound on the value of the evidence (since the evidence can only decrease in the transition region) which is, anyhow, in a regime where the model is strongly disfavoured. Moreover, one should also keep in mind that we are close to a regime where the numerical calculations cannot really be trusted (light red shaded region).

The case $\alpha<1$ is very similar and in Fig. 5, we have represented different numerical values of the Bayes factor versus $-\ell$ (blue solid line). The interpretation is very similar and one notices that, this time, one can track the evidence until the end of the transition regime, i.e. until the GMSSMI $\mathrm{omB}_{\mathrm{B}}$ model. Then, one can extrapolate it using again the ratio of the prior volumes and this leads to the following expression

$$
\ln \left(\frac{\mathcal{E}_{\log (1-\alpha) \in[-28,-\ell]}}{\mathcal{E}_{\mathrm{HI}}}\right)=\ln \left(\frac{\mathcal{E}_{\mathrm{GMSSMI}_{\mathrm{omB}}}}{\mathcal{E}_{\mathrm{HI}}}\right)+\ln \left(28-\ell_{\mathrm{c}}^{\max }\right)-\ln (28-\ell) .
$$

This expression is plotted as the (upper) dotted red line in Fig. 5 and allows us to extrapolate, in a reliable way, the Bayes factor for values $-\ell \gtrsim-20$. Let us also notice that, in this case, the calculation is performed in a regime where numerical calculations are trustful.

In Fig. 5, one also notices that, for $\alpha>1$, the evidence decreases in the transition region while, for $\alpha<1$, it grows. This is because the spectral index of GMSSMI decreases with the value of $\alpha$ starting from the MSSMI value $n_{\mathrm{S}} \simeq 0.9$ when $|\alpha-1| \simeq 0$. As a consequence, when $\alpha<1$, if $-\ell$ is increased then $n_{\mathrm{S}}$ grows and, therefore, crosses the Planck best fit region. For this reason, the blue curve in Fig. 5 increases in the transition region. In the case $\alpha>1$, one observes the opposite behaviour since, in that situation, the model moves away from the Planck best fit region.

The previous considerations allow us to extrapolate the evidence analytically to the theoretical prior in which $\alpha$ varies up to unity. Those two extrapolated models have been named $\mathrm{GMSSMI}_{\mathrm{ep}}$ and GMSSMI $\mathrm{em}_{\mathrm{em}}$ in the next table and their evidence have been estimated

\begin{tabular}{|c|c|c|c|c|c|}
\hline Name & Priors & $\ln \left(\mathcal{E} / \mathcal{E}_{\mathrm{HI}}\right)$ & $\mathcal{C}$ & $\bar{N}$ & $\ln \left(\mathcal{L}_{\max } / \mathcal{E}_{\mathrm{HI}}\right)$ \\
\hline GMSSMI $_{\mathrm{ep}}$ & $\begin{array}{c}\phi_{0} / M_{\mathrm{Pl}} \in\left[2 \times 10^{-5}, 2 \times 10^{-4}\right] \\
\log (\alpha-1) \in[-28,-0] \\
\ln R \in[-46,0]\end{array}$ & -11.69 & 3.00 & 4 & -7.40 \\
\hline GMSSMI $_{\mathrm{em}}$ & $\begin{array}{c}\phi_{0} / M_{\mathrm{Pl}} \in\left[2 \times 10^{-5}, 2 \times 10^{-4}\right] \\
\log (1-\alpha) \in[-28,-0] \\
\ln R \in[-46,0]\end{array}$ & -6.53 & 3.88 & 4 & 2.27 \\
\hline
\end{tabular}
using the two equations derived above, namely Eqs. (A.53) and (A.54).

In the above table, the complexities have been rescaled following the rough estimate given by Eq. (A.8). 


\section{A.31 Generalised Renormalisable Point Inflation (GRIPI)}

In the very same way as GMSSMI is a generalisation of MSSMI, see section A.30, the GRIPI potential is a generalisation of the RIPI one, see section A.19. This potential can be written as

$$
V(\phi)=M^{4}\left[\left(\frac{\phi}{\phi_{0}}\right)^{2}-\frac{4 \alpha}{3}\left(\frac{\phi}{\phi_{0}}\right)^{3}+\frac{\alpha}{2}\left(\frac{\phi}{\phi_{0}}\right)^{4}\right],
$$

and depends on two parameters, $\phi_{0}$ and $\alpha$. The case $\alpha=1$ corresponds to the RIPI potential. As discussed in Ref. [5], the typical value of the vev $\phi_{0}$ is given by $\phi_{0} \simeq 10^{14} \mathrm{GeV}$ and/or $\phi_{0} \simeq 10^{17} \mathrm{GeV}$. In fact, in the case $\phi_{0} \simeq 10^{14} \mathrm{GeV}$, the amount of fine-tuning is similar to the GMSSMI case. For this reason, it is not so interesting to replicate the discussion of the previous section and, here, one focuses on the case $\phi_{0} \simeq 10^{17} \mathrm{GeV}$ where one can expect the fine tuning problem to be sligthly less severe.

Let us now discuss the priors. The GRIPI potential can always be viewed as a phenomenological model (simply denoted GRIPI $_{p}$ in what follows). In that case, the order of magnitude of the parameter $\phi_{0}$ is not specified and, therefore, one chooses a Jeffreys prior, namely $\log \left(\phi_{0} / M_{\mathrm{P} 1}\right) \in[-5,5]$. Regarding the parameter $\alpha$, since it is of order one, we simply take $\alpha \in[0.9,1.1]$. As for GMSSMI, we have also added a hard prior boundary, enforcing $\left|\epsilon_{2}\right|<0.2$, as otherwise some regions of the parameter space would predict non-slow-roll inflation.

Returning to the original version of the model and considering the fact that, in this case, the vev $\phi_{0}$ is specified, we choose the prior $\phi_{0} / M_{\mathrm{Pl}} \in\left[2 \times 10^{-2}, 2 \times 10^{-1}\right]$. As for GMSSMI, if $\alpha$ is not tuned around $\alpha=1$, the model becomes inconsistent. Requiring at least $\Delta N \simeq 60$ $e$-fold during inflation leads to the condition

$$
|\alpha-1|<\frac{\phi_{0}^{4}}{M_{\mathrm{P} 1}^{4}} \frac{\pi^{2}}{576 \Delta N^{2}},
$$

see Ref. [5] and, therefore, if $|\alpha-1| \gtrsim 10^{-8}$, then the model is a priori ruled out. As a consequence, when $\alpha>1$, we consider two cases satisfying the above constraint namely $\log (1-\alpha) \in[-15,-10]$ and $\log (1-\alpha) \in[-28,-8]$, thus defining the GRIPI omA $_{\text {and GRIPI }}$ omB models. If $\alpha>1$, we define two other models denoted GRIPI $\mathrm{opA}_{\mathrm{o} A}$ and GRIPI $\mathrm{opB}_{\mathrm{oB}}$ such that $\log (\alpha-1) \in[-28,-10]$ and $\log (\alpha-1) \in[-28,-8]$.

Finally, the GRIPI potential can also arise in a supergravity framework (we denote this

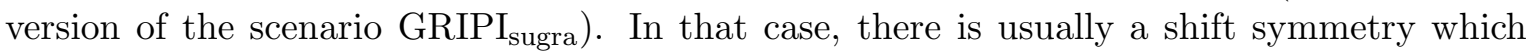
allows us to consider super-Planckian vev of the field. For this reason, we also investigate the prior $\phi_{0} / M_{\mathrm{Pl}} \in[10,50]$. The prior on $\alpha$ is still taken to be with $\alpha \in[0.9,1.1]$ in agreement with the previous discussion. All the considerations presented in this section are summarised in the table below. 


\begin{tabular}{|c|c|c|c|c|c|}
\hline Name & Priors & $\ln \left(\mathcal{E} / \mathcal{E}_{\mathrm{HI}}\right)$ & $\mathcal{C}$ & $N$ & $\ln \left(\mathcal{L}_{\max } / \mathcal{E}_{\mathrm{HI}}\right)$ \\
\hline GRIPI $_{p}$ & $\begin{array}{c}\log \left(\phi_{0} / M_{\mathrm{Pl}}\right) \in[-5,5] \\
\alpha \in[0.9,1.1]\end{array}$ & -2.77 & 4.00 & 4 & 2.29 \\
\hline $\mathrm{GRIPI}_{\mathrm{opA}}$ & $\begin{array}{c}\phi_{0} / M_{\mathrm{Pl}} \in\left[2 \times 10^{-2}, 2 \times 10^{-1}\right] \\
\log (\alpha-1) \in[-15,-10] \\
\ln R \in[-46,0], \Delta N>60\end{array}$ & -5.10 & 3.14 & 4 & -0.31 \\
\hline $\mathrm{GRIPI}_{\mathrm{opB}}$ & $\begin{array}{c}\phi_{0} / M_{\mathrm{Pl}} \in\left[2 \times 10^{-2}, 2 \times 10^{-1}\right] \\
\log (\alpha-1) \in[-15,-8] \\
\ln R \in[-46,0], \Delta N>60\end{array}$ & -5.39 & 3.21 & 4 & -0.31 \\
\hline GRIPI $_{\mathrm{omA}}$ & $\begin{array}{c}\phi_{0} / M_{\mathrm{Pl}} \in\left[2 \times 10^{-2}, 2 \times 10^{-1}\right] \\
\log (1-\alpha) \in[-15,-10] \\
\ln R \in[-46,0], \Delta N>60\end{array}$ & -4.60 & 6.42 & 4 & 1.87 \\
\hline GRIPI $_{\mathrm{omB}}$ & $\begin{array}{c}\phi_{0} / M_{\mathrm{Pl}} \in\left[2 \times 10^{-2}, 2 \times 10^{-1}\right] \\
\log (1-\alpha) \in[-15,-8] \\
\ln R \in[-46,0], \Delta N>60\end{array}$ & -4.30 & 5.72 & 4 & 1.99 \\
\hline GRIPI $_{\text {sugra }}$ & $\begin{array}{c}\phi_{0} / M_{\mathrm{P} 1} \in[10,50] \\
\alpha \in[0.9,1.1]\end{array}$ & -0.96 & 2.96 & 4 & 2.23 \\
\hline
\end{tabular}

As was done in the case of GMSSM inflation in the previous section, one can also study how the choice of the prior on $\alpha$ affects the determination of the Bayesian evidence. For this reason, we consider the following priors: $\log (\alpha-1) \in[-15,-\ell]$ for $\alpha>1$ and $\log (1-\alpha) \in[-15,-\ell]$ for $\alpha<1$. The dependence of the evidence with respect to $\ell$ can be derived as in the previous section. In the prior plane $\left[\phi_{0} / M_{\mathrm{Pl}}, \log |1-\alpha|\right]$, Eq. (A.56) defines a line above which the likelihood is tiny and can be considered to be vanishing. This line divides the prior space into two parts and goes from $\left(2 \times 10^{-2},-12 \equiv \ell_{\mathrm{c}}^{\mathrm{min}}\right)$ to $\left(2 \times 10^{-1},-8 \equiv \ell_{\mathrm{c}}^{\max }\right)$ and, therefore, defines three different regions according to whether $-\ell<\ell_{\mathrm{c}}^{\min },-\ell \in\left[\ell_{\mathrm{c}}^{\min }, \ell_{\mathrm{c}}^{\max }\right]$ or $-\ell>\ell_{\mathrm{c}}^{\max }$.

Let us first assume that $\alpha>1$. If $-\ell \lesssim-15$, then $\alpha-1$ is tiny and one expects GRIPI to be equivalent to RIPI (with the same value of $\phi_{0}$ ). If $\ell \in\left[\ell_{\mathrm{c}}^{\min }, \ell_{\mathrm{c}}^{\max }\right]$, then only numerical calculations can track the behaviour of the evidence. Notice that GRIPI $_{\mathrm{opA}}$ and GRIPI $_{\mathrm{opB}}$ belongs to this region. Finally, for $-\ell>\ell_{\mathrm{c}}^{\max }$, one expects the evidence to scale with the ratio of the prior volumes. In that case, one can write

$$
\ln \left(\frac{\mathcal{E}_{\log (\alpha-1) \in[-15,-\ell]}}{\mathcal{E}_{\mathrm{HI}}}\right)=\ln \left(\frac{\mathcal{E}_{\mathrm{GRIPI}}}{\mathcal{E}_{\mathrm{HI}}}\right)+\ln \left(15-\ell_{\mathrm{c}}^{\max }\right)-\ln (15-\ell) .
$$

Here, we have taken GRIPI ${ }_{\mathrm{opB}}$ as the calibration model, a natural choice considering that this model lies at the frontier of the transition region. The corresponding results are represented in Fig. 6 (solid green line for the numerical results and dashed red line for the extrapolated evidences).

For $\alpha<1$, taking GRIPI $\mathrm{omB}_{\mathrm{om}}$ as a calibration model, exactly the same discussion applies and one is led to (again, see Fig. 6)

$$
\ln \left(\frac{\mathcal{E}_{\log (1-\alpha) \in[-15,-\ell]}}{\mathcal{E}_{\mathrm{HI}}}\right)=\ln \left(\frac{\mathcal{E}_{\mathrm{GRIPI}} \mathcal{E}_{\mathrm{HI}}}{\mathcal{H}_{\mathrm{om}}}\right)+\ln \left(15-\ell_{\mathrm{c}}^{\max }\right)-\ln (15-\ell) .
$$

We can now use these formulae to rescale the evidence if $\alpha$ varies up to unity. Naming the two corresponding models GRIPI $\mathrm{ep}_{\mathrm{ep}}$ and $\mathrm{GRIPI}_{\mathrm{em}}$, their evidences have been reported below. 


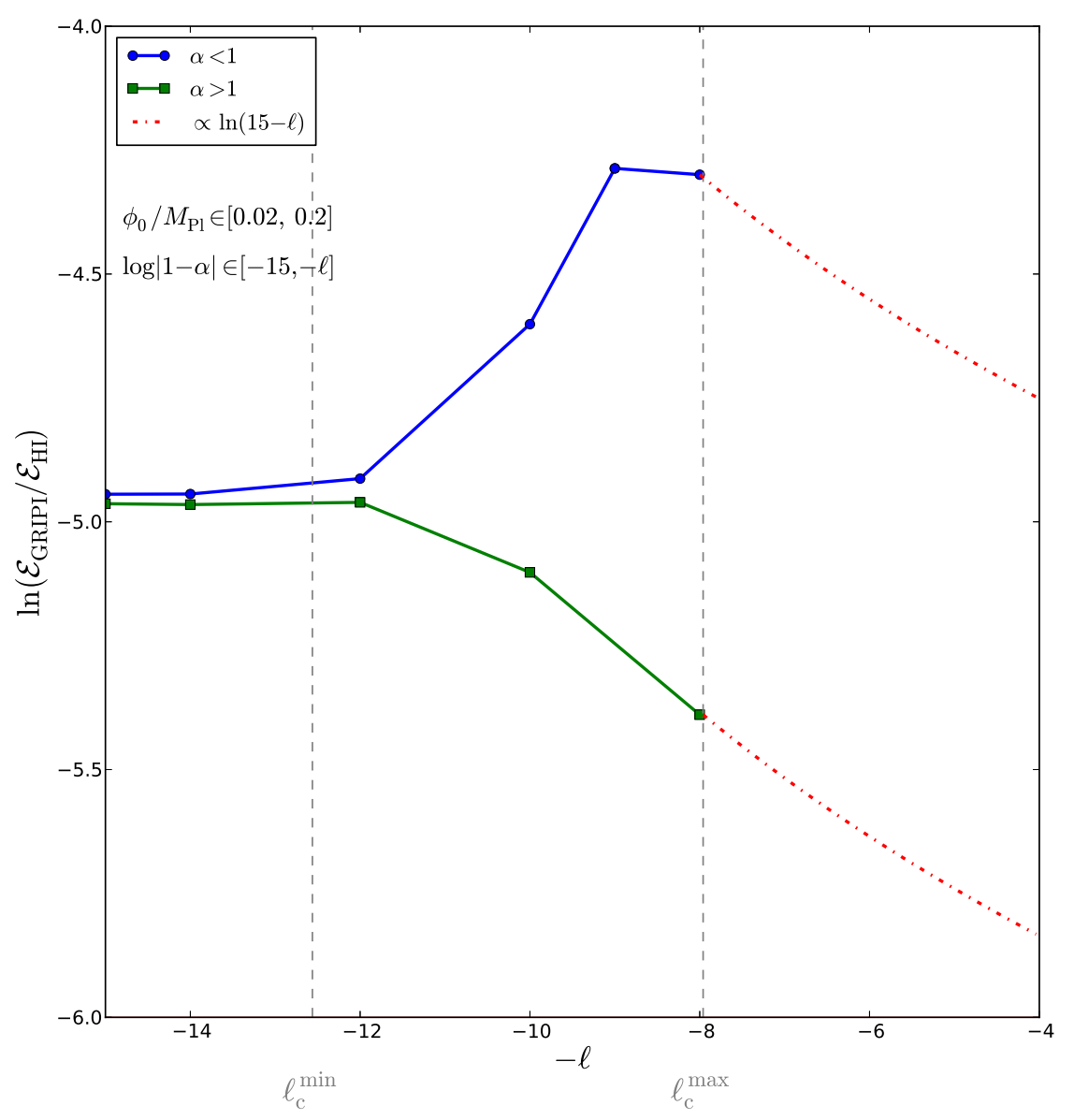

Figure 6. Evolution of the GRIPI Bayes factor versus the upper bound $-\ell$ of the prior range on $\alpha$ for $\alpha>1$ and $\alpha<1$. The green squares and blue circles represent numerical values of the evidence. The dotted red curves represent the analytical laws giving the behaviour of the Bayes factor versus $-\ell$ for $-\ell \gtrsim \ell_{\mathrm{c}}^{\max }$ according to Eqs. (A.57) and (A.58). These equations predict how the Bayes factor behaves with $-\ell$ and, therefore, can be used to extrapolate in regimes where $\alpha$ becomes of order one. The behaviour of the evidences is very similar to what was found in the GMSSMI case, see Fig. 5 . However, a difference with GMSSMI is that, in the case $\alpha>1$, one is now able to track the Bayes factors through the entire transition region.

\begin{tabular}{|l||c||c||c||c||c|}
\hline Name & Priors & $\ln \left(\mathcal{E} / \mathcal{E}_{\mathrm{HI}}\right)$ & $\mathcal{C}$ & $N$ & $\ln \left(\mathcal{L}_{\mathrm{max}} / \mathcal{E}_{\mathrm{HI}}\right)$ \\
\hline GRIPI $_{\mathrm{ep}}$ & $\begin{array}{c}\phi_{0} / M_{\mathrm{Pl}} \in\left[2 \times 10^{-2}, 2 \times 10^{-1}\right] \\
\log (\alpha-1) \in[-15,0]\end{array}$ & -6.15 & 3.79 & 4 & -0.31 \\
\hline GRIPI $_{\mathrm{em}}$ & $\begin{array}{c}\phi_{0} / M_{\mathrm{Pl}} \in\left[2 \times 10^{-2}, 2 \times 10^{-1}\right] \\
\log (1-\alpha) \in[-15,0]\end{array}$ & -5.06 & 4.28 & 4 & 1.99 \\
\hline
\end{tabular}

In this table, complexities have also been rescaled following the rough estimate given by Eq. (A.8). 


\section{A.32 Brane SUSY Breaking Inflation (BSUSYBI)}

The potential is a sum of two exponential and reads

$$
V(\phi)=M^{4}\left(e^{\sqrt{6} \frac{\phi}{M_{\mathrm{Pl}}}}+e^{\sqrt{6} \gamma \frac{\phi}{M_{\mathrm{P} 1}}}\right) .
$$

In addition to the parameter $\gamma$, the field value $x_{\mathrm{end}}=\phi_{\mathrm{end}} / M_{\mathrm{P} 1}$ at which inflation ends has to be specified. Within the superstring scenario from which this model is inspired, $0<\gamma<1 / \sqrt{3}$ [69]. However, the upper limit would already implies to $\epsilon_{1}(x)>3 \gamma^{2} \simeq 1$ and slow-roll is violated everywhere. We have therefore limited the prior on $\gamma$ to slightly lower values $\gamma<0.3$ considering either a flat prior or a Jeffreys prior. Concerning, $x_{\text {end }}$, one notices that inflation proceeds at decreasing field values and is confined in a region $x<x_{\epsilon_{1}=1}$, $x_{\epsilon_{1}=1}$ being the solution of $\epsilon_{1}(x)=1$. As a result, there is a maximal bound $x_{\text {end }}^{\max }$ which has been defined such that inflation last more than $120 e$-folds. The quantity is only known numerically and is obtained by integrating the field trajectory from $x_{\mathrm{ini}}=x_{\epsilon_{1}=1}$ during 120 $e$-folds. On the contrary, there is no lower limit on the allowed values of $x_{\text {end }}$ and the limit $x_{\text {end }} \rightarrow-\infty$, would correspond to $n_{\mathrm{S}}=1$ and $r=0$. Therefore, for $x_{\text {end }}$ negative enough, the likelihood, and therefore the evidence, becomes independent on the lower bound on $x_{\text {end }}$. We have therefore considered the following priors:

\begin{tabular}{|l||c||c||c||c||c|}
\hline Name & Priors & $\ln \left(\mathcal{E} / \mathcal{E}_{\mathrm{HI}}\right)$ & $\mathcal{C}$ & $N$ & $\ln \left(\mathcal{L}_{\mathrm{max}} / \mathcal{E}_{\mathrm{HI}}\right)$ \\
\hline BSUSYBI $_{\mathrm{f}}$ & $\begin{array}{c}\gamma \in[0,0.3] \\
x_{\text {end }} \in\left[-200, x_{\mathrm{end}}^{\max }\right]\end{array}$ & 3.26 & 3.26 & 4 & -2.54 \\
\hline BSUSYBI $_{1}$ & $\begin{array}{c}\log (\gamma) \in[-3,-1] \\
x_{\text {end }} \in\left[-200, x_{\mathrm{end}}^{\max }\right]\end{array}$ & 4.52 & 3.26 & 4 & -2.54 \\
\hline
\end{tabular}

\section{A.33 Tip Inflation (TI)}

This string inspired potential has two parameters, a dimensionless coupling $\alpha$ and a typical vev $\mu$ :

$$
V(\phi)=M^{4}\left[1+\cos \left(\frac{\phi}{\mu}\right)+\alpha \sin ^{2}\left(\frac{\phi}{\mu}\right)\right] .
$$

As made explicit in Ref. [5], these parameters encode combinations of geometrical quantities related to the relative position of branes within a conifold geometry. This potential supports inflation at its top provided $\alpha \simeq 1 / 2$, which amounts to some level of fine-tuning. When this condition is satisfied, $\mu$ actually gives the volume of the extra-dimensions

$$
\frac{\mu}{M_{\mathrm{P} 1}} \simeq 2 \times 10^{8} \sigma_{0}^{9 / 4}
$$

where $\sigma_{0}$ is the stabilised value of the volume modulus in the absence of uplifting terms [70]. A typical value for $\sigma_{0}$ is $\sigma_{0} \simeq 10^{2}$ which translates into $\mu / M_{\mathrm{P} 1} \simeq 10^{-4}$, up to a few orders of magnitude. Following these considerations, we have examined various priors designed to measure how important is the fine-tuning over $\alpha$. In particular, the three sub-classes $\alpha \gtrsim 1 / 2, \alpha=1 / 2$ and $\alpha \lesssim 1 / 2$ yield different observable predictions and have been treated as separated models. They are summarised below. 


\begin{tabular}{|l||c||c||c||c||c|}
\hline Name & Priors & $\ln \left(\mathcal{E} / \mathcal{E}_{\mathrm{HI}}\right)$ & $\mathcal{C}$ & $N$ & $\ln \left(\mathcal{L}_{\max } / \mathcal{E}_{\mathrm{HI}}\right)$ \\
\hline $\mathrm{TI}_{\alpha>1 / 2}^{\mathrm{ft}+}$ & $\begin{array}{c}\left.\alpha \in] 0.5,0.5+10^{-7}\right] \\
\log \left(\mu / M_{\mathrm{P} 1}\right) \in[-5,-3]\end{array}$ & -4.37 & 3.51 & 4 & 1.95 \\
\hline $\mathrm{TI}_{\alpha>1 / 2}^{\mathrm{ft}}$ & $\begin{array}{c}\left.\alpha \in] 0.5,0.5+2 \times 10^{-7}\right] \\
\log \left(\mu / M_{\mathrm{Pl}}\right) \in[-5,-3]\end{array}$ & -5.19 & 3.53 & 4 & 1.95 \\
\hline $\mathrm{TI}_{\alpha>1 / 2}^{\mathrm{ft}-}$ & $\begin{array}{c}\left.\alpha \in] 0.5,0.5+10^{-6}\right] \\
\log \left(\mu / M_{\mathrm{P} 1}\right) \in[-5,-3]\end{array}$ & -6.99 & 3.51 & 4 & 1.95 \\
\hline $\mathrm{TI}_{1 / 2}$ & $\begin{array}{c}\alpha=1 / 2 \\
\log \left(\mu / M_{\mathrm{P} 1}\right) \in[-5,-3]\end{array}$ & -1.90 & 2.82 & 3 & 1.95 \\
\hline $\mathrm{TI}_{\alpha<1 / 2}^{\mathrm{ft}+}$ & $\begin{array}{c}\alpha \in\left[0.5-10^{-7}, 0.5[\right. \\
\log \left(\mu / M_{\mathrm{P} 1}\right) \in[-5,-3]\end{array}$ & -1.92 & 2.59 & 4 & 2.21 \\
\hline $\mathrm{TI}_{\alpha<1 / 2}^{\mathrm{ft}}$ & $\begin{array}{c}\alpha \in\left[0.5-10^{-6}, 0.5[\right. \\
\log \left(\mu / M_{\mathrm{Pl}}\right) \in[-5,-3]\end{array}$ & -4.64 & 2.60 & 4 & 2.21 \\
\hline $\mathrm{TI}_{\alpha<1 / 2}^{\mathrm{ft}-}$ & $\begin{array}{c}\alpha \in\left[0.5-10^{-5}, 0.5[\right. \\
\log \left(\mu / M_{\mathrm{Pl}}\right) \in[-5,-3]\end{array}$ & -7.12 & 2.59 & 4 & 2.21 \\
\hline
\end{tabular}

For completeness, we have also considered models in which $\alpha \simeq 1 / 2$ without any prior prejudice on the sign of $\alpha-1 / 2$. One gets the following evidences:

\begin{tabular}{|l||c||c||c||c||c|}
\hline Name & Priors & $\ln \left(\mathcal{E} / \mathcal{E}_{\mathrm{HI}}\right)$ & $\mathcal{C}$ & $N$ & $\ln \left(\mathcal{L}_{\mathrm{max}} / \mathcal{E}_{\mathrm{HI}}\right)$ \\
\hline $\mathrm{TI}_{\mathrm{ft}+}$ & $\begin{array}{c}\alpha \in\left[0.5-10^{-7}, 0.5+10^{-7}\right] \\
\log \left(\mu / M_{\mathrm{Pl}}\right) \in[-5,-3]\end{array}$ & -2.65 & 2.74 & 4 & 2.21 \\
\hline $\mathrm{TI}_{\mathrm{ft}}$ & $\begin{array}{c}\alpha \in\left[0.5-10^{-6}, 0.5+10^{-6}\right] \\
\log \left(\mu / M_{\mathrm{Pl}}\right) \in[-5,-3]\end{array}$ & -5.26 & 2.74 & 4 & 2.21 \\
\hline $\mathrm{TI}_{\mathrm{ft}-}$ & $\begin{array}{c}\alpha \in\left[0.5-10^{-5}, 0.5+10^{-5}\right] \\
\log \left(\mu / M_{\mathrm{Pl}}\right) \in[-5,-3]\end{array}$ & -7.78 & 2.75 & 4 & 2.21 \\
\hline
\end{tabular}

One can also wonder what happens if one detunes the prior on $\alpha$ since, after all, this fine-tuning is not theoretically motivated. Let us first consider the case where $\alpha>1 / 2$. We want to calculate the evidence if the prior on $\alpha$ is chosen such that $\alpha \in] 0.5,0.5+a]$. We assume that, for $a>10^{-6}$, the likelihood vanishes (which is, according to the results presented in the tables, a realistic hypothesis). Applying the considerations presented earlier [see Eq. (A.4)] and taking as a calibration model $\mathrm{TI}_{\alpha>1 / 2}^{\mathrm{ft}-}$, one obtains that for $a>10^{-6}$

$$
\mathcal{E}_{\alpha \in] 0.5,0.5+a]}=\mathcal{E}_{\left.\alpha \in] 0.5,0.5+10^{-6}\right]} \frac{10^{-6}}{a},
$$

or

$$
\ln \left(\frac{\mathcal{E}_{\alpha \in] 0.5,0.5+a]}}{\mathcal{E}_{\mathrm{HI}}}\right)=\ln \left(\frac{\mathcal{E}_{\mathrm{TI}}^{\mathrm{ft}-}}{\mathcal{E}_{\mathrm{HI}}}\right)-6 \ln (10)-\ln (a) \simeq-6.99-6 \ln (10)-\ln (a) .
$$

If we now assume $\alpha<1 / 2$, the same considerations lead to a similar formula, namely

$$
\ln \left(\frac{\mathcal{E}_{\alpha \in] 0.5-a, 0.5]}}{\mathcal{E}_{\mathrm{HI}}}\right)=\ln \left(\frac{\mathcal{E}_{\mathrm{TI}}^{\mathrm{ft}-}-}{\mathcal{E}_{\mathrm{HI}}}\right)-5 \ln (10)-\ln (a) \simeq-7.12-5 \ln (10)-\ln (a),
$$

for $a>10^{-5}$. Finally, the case where the sign of $\alpha-1 / 2$ is not specified yields

$$
\ln \left(\frac{\mathcal{E}_{\alpha \in] 0.5-a, 0.5+a]}}{\mathcal{E}_{\mathrm{HI}}}\right)=\ln \left(\frac{\mathcal{E}_{\mathrm{TI}_{\mathrm{ft}-}}}{\mathcal{E}_{\mathrm{HI}}}\right)-5 \ln (10)-\ln (a) \simeq-7.78-5 \ln (10)-\ln (a),
$$


for $a>10^{-5}$. As expected, we see on these last three formulae that, if one increases the range of the prior in a region where the likelihood vanishes, then the corresponding models get penalised for the wasted parameter space. Therefore, the above calculations concretely illustrate the Occam's razor effect.

Applying these formulae allows us to extrapolate the evidence for a natural prior choice having $a=10^{-1}$, i.e. assuming only $|\alpha-0.5|<0.1$.

\begin{tabular}{|l||c||c||c||c||c|}
\hline Name & Priors & $\ln \left(\mathcal{E} / \mathcal{E}_{\mathrm{HI}}\right)$ & $\mathcal{C}$ & $N$ & $\ln \left(\mathcal{L}_{\mathrm{max}} / \mathcal{E}_{\mathrm{HI}}\right)$ \\
\hline $\mathrm{TI}_{\alpha<1 / 2}^{\mathrm{e}}$ & $\begin{array}{c}\alpha \in[0.4,0.5] \\
\log \left(\mu / M_{\mathrm{Pl}}\right) \in[-5,-3]\end{array}$ & -16.33 & 4.00 & 4 & 2.21 \\
\hline $\mathrm{TI}_{\alpha>1 / 2}^{\mathrm{e}}$ & $\begin{array}{c}\alpha \in[0.5,0.6] \\
\log \left(\mu / M_{\mathrm{Pl}}\right) \in[-5,-3]\end{array}$ & -18.50 & 4.00 & 4 & 1.95 \\
\hline $\mathrm{TI}^{\mathrm{e}}$ & $\begin{array}{c}\alpha \in[0.4,0.6] \\
\log \left(\mu / M_{\mathrm{Pl}}\right) \in[-5,-3]\end{array}$ & -16.99 & 4.00 & 4 & 2.21 \\
\hline
\end{tabular}

In this table, complexities have also been rescaled following the rough estimate given by Eq. (A.8). Here the volume ratio is so big that the rescaled complexities end up being very close to the number of parameters, which is certainly overestimated due to our crude assumptions in deriving Eq. (A.8).

\section{A.34 Beta Exponential Inflation (BEI)}

This model is an extension of PLI to the generalised exponential function $\exp _{1-\beta}$ defined by $\exp _{1-\beta}(x)=(1+\beta x)^{1 / \beta}$ for $1+\beta x>0$ and $\exp _{1-\beta}(x)=0$ otherwise. The potential therefore reads

$$
V(\phi)=M^{4} \exp _{1-\beta}\left(\lambda \frac{\phi}{M_{\mathrm{Pl}}}\right)
$$

where $\lambda>0$ is a dimensionless parameter. As detailed in Ref. [5], inflation ends naturally only for $\beta>0$, which will be our prior. The model being phenomenological, there is no natural value for $\lambda$ and we have chosen a Jeffreys prior. Moreover, one can show that the slow-roll observable predictions does not depend on $\lambda$, and thus the prior boundaries do not affect the evidence. In the limit $\beta \rightarrow 0$, the model becomes strongly disfavoured such that, changing the lower limit of the $\beta$-prior accordingly decreases the evidence of the model. This is summarised in the following table:

\begin{tabular}{|l||c||c||c||c||c|}
\hline Name & Priors & $\ln \left(\mathcal{E} / \mathcal{E}_{\mathrm{HI}}\right)$ & $\mathcal{C}$ & $N$ & $\ln \left(\mathcal{L}_{\max } / \mathcal{E}_{\mathrm{HI}}\right)$ \\
\hline BEI & $\begin{array}{c}\log (\lambda) \in[-3,3] \\
\log (\beta) \in[-1.5,3]\end{array}$ & -0.99 & 2.65 & 4 & 2.29 \\
\hline
\end{tabular}

\section{A.35 Pseudo Natural Inflation (PSNI)}

The potential of PSNI reads

$$
V(\phi)=M^{4}\left[1+\alpha \ln \left(\cos \frac{\phi}{f}\right)\right],
$$

where $\alpha$ is a dimensionless coupling and $f$ is an energy scale. In order for the model to be consistent, one should have $f<m_{\mathrm{Pl}}=\sqrt{8 \pi} M_{\mathrm{Pl}}$ and $\alpha \ll 1$ [71]. As discussed in Ref. [5], the above potential has $\epsilon_{2}>\epsilon_{2}^{\min }=2 \alpha M_{\mathrm{Pl}}^{2} / f^{2}$ and slow-roll inflation can occur only if $\alpha M_{\mathrm{Pl}}^{2} / f^{2}$ is constrained to be small. 

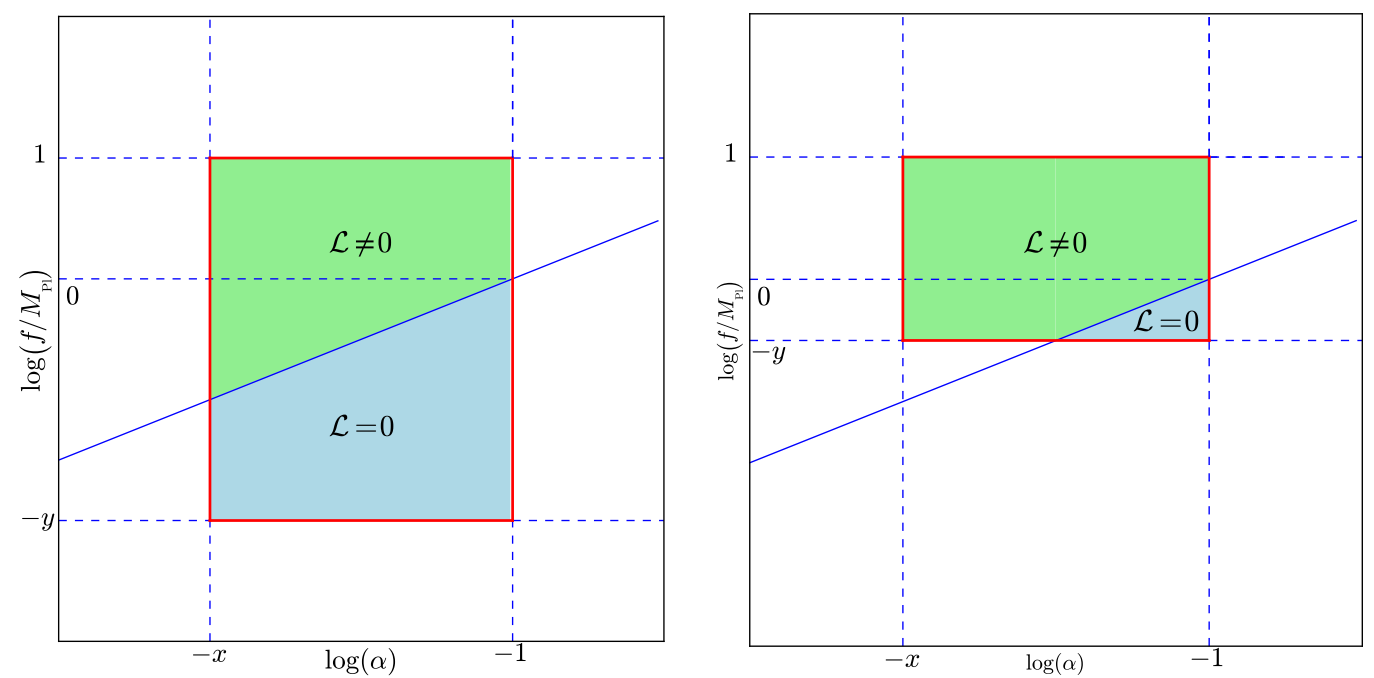

Figure 7. Priors for PSNI inflation in the plane $\left[\log \alpha, \log \left(f / M_{\mathrm{P} 1}\right)\right]$. The red rectangle represents the theoretical motivated prior. The blue line represents the condition of validity of the slow-roll approximation, namely $\alpha f^{2} / M_{\mathrm{Pl}}^{2}<0.1$. Above this line (green region), slow-roll is satisfied and below (blue region) slow-roll is strongly violated. As a consequence, the likelihood vanishes in the blue region and is different from zero in the green one. Numerically, one can only determine the Bayesian evidence with a prior corresponding to the green region. The evidence corresponding to the red rectangle can be derived from analytical considerations (see text). The left panel corresponds to the situation where $y>(x-1) / 2$ while the right panel is for $y<(x-1) / 2$.

A first phenomenological choice of priors therefore consists in adopting prior boundaries for uniform priors in the quantities $\log \left(\alpha M_{\mathrm{Pl}}^{2} / f^{2}\right)$ and $\log \left(f / M_{\mathrm{Pl}}\right)$. Taking $\log \left(f / M_{\mathrm{Pl}}\right) \in$ $[-2,1]$ and $\log \left(\alpha M_{\mathrm{Pl}}^{2} / f^{2}\right)<-1$, different lower bounds on $\alpha M_{\mathrm{Pl}}^{2} / f^{2}$ have been studied, corresponding to different levels of fine-tuning of this parameter.

\begin{tabular}{|l||c||c||c||c||c|}
\hline Name & Priors & $\ln \left(\mathcal{E} / \mathcal{E}_{\mathrm{HI}}\right)$ & $\mathcal{C}$ & $N$ & $\ln \left(\mathcal{L}_{\mathrm{max}} / \mathcal{E}_{\mathrm{HI}}\right)$ \\
\hline $\mathrm{PSNI}_{\mathrm{ft} 1}$ & $\begin{array}{c}\log \left(\alpha M_{\mathrm{Pl}}^{2} / f^{2}\right) \in[-5,-1] \\
\log \left(f / M_{\mathrm{Pl}}\right) \in[-2,1]\end{array}$ & -0.42 & 2.13 & 4 & 2.29 \\
\hline $\mathrm{PSNI}_{\mathrm{ft} 2}$ & $\begin{array}{c}\log \left(\alpha M_{\mathrm{Pl}}^{2} / f^{2}\right) \in[-3,-1] \\
\log \left(f / M_{\mathrm{Pl}}\right) \in[-2,1]\end{array}$ & -0.41 & 2.04 & 4 & 2.29 \\
\hline $\mathrm{PSNI}_{\mathrm{ft} 3}$ & $\begin{array}{c}\log \left(\alpha M_{\mathrm{Pl}}^{2} / f^{2}\right) \in[-2,-1] \\
\log \left(f / M_{\mathrm{Pl}}\right) \in[-2,1]\end{array}$ & -0.64 & 1.74 & 4 & 2.29 \\
\hline $\mathrm{PSNI}_{\mathrm{ft} 4}$ & $\begin{array}{c}\log \left(\alpha M_{\mathrm{Pl}}^{2} / f^{2}\right) \in[-1.5,-1] \\
\log \left(f / M_{\mathrm{Pl}}\right) \in[-2,1]\end{array}$ & -5.62 & 3.68 & 4 & 0.02 \\
\hline
\end{tabular}

One can see that the evidence increases as the lower bound on $\log \left(\alpha M_{\mathrm{Pl}}^{2} / f^{2}\right)$ decreases because the likelihood is better in the region where $\log \left(\alpha M_{\mathrm{Pl}}^{2} / f^{2}\right)$ is small.

Another sensible choice of priors, based on the previous considerations, is to take uniform priors on the theoretical motivated parameter $\log (\alpha) \in[-x,-1]$ and $\log \left(f / M_{\mathrm{Pl}}\right) \in$ $[-y, 1]$, where $x$ and $y$ are positive numbers left unspecified for the moment. In the plane $\left[\log \left(f / M_{\mathrm{Pl}}\right), \log \alpha\right]$ the two-dimensional prior range is represented in Fig. 7 by the red rectangle. As discussed previously, $\alpha M_{\mathrm{Pl}}^{2} / f^{2}$ must be a small quantity for slow-roll to be satisfied. In the numerical calculations, we have assumed that it is smaller than 0.1, i.e., we use the 
same hard prior boundary as before, $\epsilon_{2}<0.2$. Such a hard prior cuts the theoretical prior domain of figure 7 along the curve

$$
\log \left(\frac{f}{M_{\mathrm{Pl}}}\right)>\frac{1}{2} \log \alpha+\frac{1}{2},
$$

which is represented by a blue line in Fig. 7. As for GMSSMI and GRIPI, we can estimate analytically how the evidence would be rescaled by removing this hard prior, but first we need to estimate how much prior volume is affected.

When $y \geq(x-1) / 2$ (left panel of Fig. 7 ), the hard prior boundary line intersects the right vertical edge of the red rectangle at the point $(-1,0)$ and the left vertical edge at $(-x, 1 / 2-x / 2)$. In the case where $y \leq(x-1) / 2$ (right panel of Fig. 7$)$, this line still intersects the right vertical edge of the red rectangle at the point $(-1,0)$ but now meets the bottom horizontal edge at $(-1-2 y,-y)$. The condition (A.68) corresponds to the green region in Fig. 7, where slow-roll is valid and the likelihood non-vanishing. The complementary domain has been represented in blue on the same figure. In this domain, slow-roll is violated and the predictions cannot be in agreement with the observations. As a consequence, the likelihood function $\mathcal{L}$ is very small and for the purpose of our analytical extrapolation it will be assumed to vanish. In the following table, we have numerically computed the evidences in the green domain, i.e. in the region where our computations can be trusted, for various prior choices.

\begin{tabular}{|l||c||c||c||c||c|}
\hline Name & Priors & $\ln \left(\mathcal{E} / \mathcal{E}_{\mathrm{HI}}\right)$ & $\mathcal{C}$ & $N$ & $\ln \left(\mathcal{L}_{\max } / \mathcal{E}_{\mathrm{HI}}\right)$ \\
\hline \multirow{2}{*}{ PSNI $_{\mathrm{OA}}$} & $\begin{array}{c}\log (\alpha) \in[-7,-1] \\
\log \left(f / M_{\mathrm{Pl}}\right) \in[-2,1] \\
\alpha M_{\mathrm{Pl}}^{2} / f^{2}<10^{-1}\end{array}$ & -0.40 & 2.15 & 4 & 2.29 \\
\hline \multirow{2}{*}{$\mathrm{PSNI}_{\mathrm{OB}}$} & $\begin{array}{c}\log (\alpha) \in[-5,-1] \\
\log \left(f / M_{\mathrm{Pl}}\right) \in[-2,1] \\
\alpha M_{\mathrm{Pl}}^{2} / f^{2}<10^{-1}\end{array}$ & -0.42 & 2.12 & 4 & 2.29 \\
\hline \multirow{2}{*}{$\mathrm{PSNI}_{\mathrm{OC}}$} & $\begin{array}{c}\log (\alpha) \in[-3,-1] \\
\log \left(f / M_{\mathrm{Pl}}\right) \in[-2,1] \\
\alpha M_{\mathrm{Pl}}^{2} / f^{2}<10^{-1}\end{array}$ & -0.43 & 2.12 & 4 & 2.29 \\
\hline
\end{tabular}

We can now use these evidences calculated with the green domain prior and rescale them appropriately to obtain the evidences over the full domain, including the slow-roll violating regions, that is to say in the red rectangle. From Eq. (A.4), generalised to a two-dimensional prior, one gets

$$
\mathcal{E}_{\text {red }}=\frac{\int_{\text {green }} \mathrm{d} \log (\alpha) \mathrm{d} \log \left(f / M_{\mathrm{Pl}}\right)}{\int_{\text {red }} \mathrm{d} \log (\alpha) \mathrm{d} \log \left(f / M_{\mathrm{Pl}}\right)} \mathcal{E}_{\text {green }},
$$

i.e. the evidence is rescaled according to the ratio of the prior volumes between the green and red domains. Explicitly, one gets

$$
\int_{\text {red }} \mathrm{d} \log (\alpha) \mathrm{d} \log \left(f / M_{\mathrm{Pl}}\right)=(x-1)(y+1),
$$

and

$$
\int_{\text {green }} \mathrm{d} \log (\alpha) \mathrm{d} \log \left(f / M_{\mathrm{P} 1}\right)=\left\{\begin{array}{ccc}
\frac{(x-1)(x+3)}{4} & \text { if } & y \geq \frac{x-1}{2}, \\
(y+1)(x-1)-y^{2} & \text { if } \quad y \leq \frac{x-1}{2}
\end{array}\right.
$$


such that

$$
\ln \left(\frac{\mathcal{E}_{\mathrm{red}}}{\mathcal{E}_{\mathrm{HI}}}\right)=\ln \left(\frac{\mathcal{E}_{\text {green }}}{\mathcal{E}_{\mathrm{HI}}}\right)+\left\{\begin{array}{r}
\ln \left[\frac{x+3}{4(y+1)}\right] \quad \text { if } \quad y \geq \frac{x-1}{2}, \\
\ln \left[1-\frac{y^{2}}{(y+1)(x-1)}\right] \quad \text { if } \quad y \leq \frac{x-1}{2} .
\end{array}\right.
$$

Therefore, the rescaled evidence (for the red domain) can be obtained from the one in the green region by using the correction factor given by the above formula. The results are summarised below.

\begin{tabular}{|l||c||c||c||c||c|}
\hline Name & Priors & $\ln \left(\mathcal{E} / \mathcal{E}_{\mathrm{HI}}\right)$ & $\mathcal{C}$ & $N$ & $\ln \left(\mathcal{L}_{\mathrm{max}} / \mathcal{E}_{\mathrm{HI}}\right)$ \\
\hline PSNI $_{\mathrm{epA}}$ & $\begin{array}{c}\log (\alpha) \in[-7,-1] \\
\log \left(f / M_{\mathrm{Pl}}\right) \in[-2,1]\end{array}$ & -0.65 & 2.39 & 4 & 2.29 \\
\hline $\mathrm{PSNI}_{\mathrm{epB}}$ & $\begin{array}{c}\log (\alpha) \in[-5,-1] \\
\log \left(f / M_{\mathrm{Pl}}\right) \in[-2,1]\end{array}$ & -0.83 & 2.51 & 4 & 2.29 \\
\hline $\mathrm{PSNI}_{\mathrm{epC}}$ & $\begin{array}{c}\log (\alpha) \in[-3,-1] \\
\log \left(f / M_{\mathrm{Pl}}\right) \in[-2,1]\end{array}$ & -1.13 & 2.77 & 4 & 2.29 \\
\hline
\end{tabular}

In this table, complexities have also been rescaled following the rough estimate given by Eq. (A.8).

\section{A.36 Non Canonical Kähker Inflation (NCKI)}

The model has two dimensionless parameters $\alpha$ and $\beta$ and its potential reads

$$
V(\phi)=M^{4}\left[1+\alpha \ln \left(\frac{\phi}{M_{\mathrm{Pl}}}\right)+\beta\left(\frac{\phi}{M_{\mathrm{Pl}}}\right)^{2}\right] .
$$

The logarithmic term encodes loop corrections to the monomial part of the potential [72] and, therefore, natural values of $\alpha$ are such $0<\alpha \ll 1$ whereas $\beta=\mathcal{O}(1)$. As discussed in Ref. [5], for $\beta>0$, the first Hubble flow function has a maximum $\epsilon_{1}^{\max } \simeq \beta / 2(\alpha \ll 1)$ at $x_{\epsilon_{1}^{\max }} \simeq 1 / \sqrt{\beta}$, with $x \equiv \phi / M_{\mathrm{Pl}}$. Therefore, we require $\beta$ to small enough to have $\epsilon_{1}^{\max } \ll 1$ to ensure slow-roll inflation. If this condition is not satisfied, inflation could still process in the large field limit, but would be equivalent to the LFI models. A similar requirement exists for $\beta<0$ by noticing that the second Hubble flow function verifies $\epsilon_{2}^{\min }>-8 \beta(\alpha \ll 1)$, which should be less than unity. The lower limit of $|\beta|$ is arbitrary but cannot not be too small in order to maintain the hierarchy between the loop corrections and the monomial term. On purely phenomenological grounds, taking the limit $\beta \rightarrow 0$ gives back the LI potential. We have accordingly chosen the following priors:

\begin{tabular}{|l||c||c||c||c||c|}
\hline Name & Priors & $\ln \left(\mathcal{E} / \mathcal{E}_{\mathrm{HI}}\right)$ & $\mathcal{C}$ & $N$ & $\ln \left(\mathcal{L}_{\mathrm{max}} / \mathcal{E}_{\mathrm{HI}}\right)$ \\
\hline $\mathrm{NCKI}_{\beta>0}$ & $\begin{array}{c}\log (\alpha) \in[-4,-1] \\
\beta \in[0.02,0.2]\end{array}$ & -3.91 & 2.57 & 4 & -0.11 \\
\hline $\mathrm{NCKI}_{\beta<0}$ & $\begin{array}{c}\log (\alpha) \in[-4,-1] \\
\beta \in[-0.1,-0.02]\end{array}$ & -65.07 & 4.50 & 4 & -56.01 \\
\hline
\end{tabular}




\section{A.37 Constant Spectrum Inflation (CSI)}

This potential is designed to produce a scale invariant power spectrum $n_{\mathrm{S}} \simeq 1$ and reads

$$
V(\phi)=\frac{M^{4}}{\left(1-\alpha \frac{\phi}{M_{\mathrm{Pl}}}\right)^{2}},
$$

where $\alpha$ is supposed to be small. This potential requires the field value $x_{\mathrm{end}}=\phi_{\mathrm{end}} / M_{\mathrm{Pl}}$ at which inflation stops to be specified. In the branch $x=\phi / M_{\mathrm{Pl}}<1 / \alpha$, inflation proceeds at decreasing field value while it cannot start at too large initial field value $x_{\text {ini }}$. Indeed, one has $\epsilon_{1}(x)<1$ only for $x<x_{\epsilon_{1}=1}$ such that $x_{\text {ini }}$ is bounded from above $x_{\text {ini }}<x_{\epsilon_{1}=1}$. This implies that there is a maximal bound for $x_{\mathrm{end}}$, that is numerically determined by requesting inflation to last, at least, $120 e$-folds from $x_{\mathrm{ini}}=x_{\epsilon_{1}=1}$ to $x_{\mathrm{end}}=x_{\mathrm{end}}^{\max }$. A priori, there is no lower limit for $x_{\text {end }}$. However, for $x_{\mathrm{end}}^{\mathrm{min}} \rightarrow-\infty$, all the slow-roll functions vanish, $n_{\mathrm{S}} \rightarrow 1$, $r \rightarrow 0$. Therefore, the likelihood values become independent on $x_{\text {end }}^{\min }$, as is the evidence. For convenience, we have chosen $x_{\text {end }}^{\min }(\alpha)$ such that $\epsilon_{1}\left(x_{\text {end }}^{\min }\right)>10^{-16}$, the machine precision limit. Everything is summarised in the following table.

\begin{tabular}{|l||c||c||c||c||c|}
\hline Name & Priors & $\ln \left(\mathcal{E} / \mathcal{E}_{\mathrm{HI}}\right)$ & $\mathcal{C}$ & $N$ & $\ln \left(\mathcal{L}_{\mathrm{max}} / \mathcal{E}_{\mathrm{HI}}\right)$ \\
\hline CSI & $\left.\begin{array}{c}\log (\alpha) \in[-5,-1] \\
x_{\text {end }} \in\left[x_{\text {end }}^{\text {min }}, x_{\text {end }}^{\text {max }}\right.\end{array}\right]$ & -7.80 & 0.16 & 4 & -7.72 \\
\hline
\end{tabular}

\section{A.38 Orientifold Inflation (OI)}

The potential of these models has two parameters, a coupling $\alpha$ and a vev $\phi_{0}$, and reads

$$
V(\phi)=M^{4}\left(\frac{\phi}{\phi_{0}}\right)^{4}\left[\ln ^{2}\left(\frac{\phi}{\phi_{0}}\right)-\alpha\right] .
$$

As the model is motivated by super Yang-Mills orientifold theories, the vev $\phi_{0}$ should be related to the Grand Unified energy scale and the coupling $\alpha$ should be small since $\alpha=$ $\mathcal{O}\left(1 / N_{\mathrm{c}}\right), N_{\mathrm{c}} \gg 1$ being the number of colours [73]. Therefore, we have chosen the following priors.

\begin{tabular}{|l||c||c||c||c||c|}
\hline Name & Priors & $\ln \left(\mathcal{E} / \mathcal{E}_{\mathrm{HI}}\right)$ & $\mathcal{C}$ & $N$ & $\ln \left(\mathcal{L}_{\max } / \mathcal{E}_{\mathrm{HI}}\right)$ \\
\hline OI & $\begin{array}{c}\log (\alpha) \in[-3,-1] \\
\log \left(\phi_{0} / M_{\mathrm{PI}}\right) \in[-3,-1]\end{array}$ & -6.52 & 3.62 & 4 & -3.21 \\
\hline
\end{tabular}

\section{A.39 Constant $n_{\mathrm{S}}$ C Inflation (CNCI)}

This is the class "C" of potentials, according to the classification of Ref. [5], which produces a constant spectral index. The potential is parametrised by one parameter $\alpha$ and reads

$$
V(\phi)=M^{4}\left[\left(3+\alpha^{2}\right) \operatorname{coth}^{2}\left(\frac{\alpha}{\sqrt{2}} \frac{\phi}{M_{\mathrm{Pl}}}\right)-3\right] .
$$

In addition to $\alpha$, the model requires the field value $x_{\mathrm{end}} \equiv \phi_{\mathrm{end}} / M_{\mathrm{P} 1}$ at which inflation ends to be specified. These scenarios are phenomenological and motivated for $\alpha$ small. Moreover, inflation proceeds at increasing field values and there is a region at small $x=\phi / M_{\mathrm{Pl}}$ in which $\epsilon_{1}(x)>1$. As the result, $x_{\text {ini }}>x_{\epsilon_{1}=1}$, with $x_{\epsilon_{1}=1}$ the solution of $\epsilon_{1}=1$. Requesting inflation to support at least $120 \mathrm{e}$-folds from $x_{\epsilon_{1}=1}$ implies the existence of minimal value for $x_{\text {end }}>x_{\text {end }}^{\min }(\alpha)$. These considerations lead us to the following priors: 


\begin{tabular}{|c||c||c||c||c||c|}
\hline Name & Priors & $\ln \left(\mathcal{E} / \mathcal{E}_{\mathrm{HI}}\right)$ & $\mathcal{C}$ & $N$ & $\ln \left(\mathcal{L}_{\mathrm{max}} / \mathcal{E}_{\mathrm{HI}}\right)$ \\
\hline CNCI & $\begin{array}{c}\log (\alpha) \in[-5,-1] \\
x_{\text {end }} / x_{\text {end }}^{\text {min }} \in[1,10]\end{array}$ & -7.72 & 8.25 & 4 & -3.53 \\
\hline
\end{tabular}

\section{A.40 Supergravity Brane Inflation (SBI)}

The potential depends on two dimensionless parameters $\alpha$ and $\beta$ and reads

$$
V(\phi)=M^{4}\left\{1+\left[-\alpha+\beta \ln \left(\frac{\phi}{M_{\mathrm{Pl}}}\right)\right]\left(\frac{\phi}{M_{\mathrm{Pl}}}\right)^{4}\right\} .
$$

As discussed in Ref. [5], the logarithmic term comes from loop corrections that should not dominate the field dynamics. As such, the potential supports inflation in the small field region in which it is convex. For inflation to end, one requires $\alpha \geq \alpha_{\min }(\beta)$ where $\alpha_{\min }=$ $(\beta / 4)[1-\ln (\beta / 4)]$, and $\beta$ should be a small parameter. For $\alpha>\alpha_{\min }$, inflation is well defined but, at larger field values, the potential exhibits a negative minimum showing that it cannot be extended to those regions. On the other hand, for $\alpha=\alpha_{\min }$, the potential has a vanishing minimum and is well defined everywhere. We have therefore considered these two cases.

\begin{tabular}{|l||c||c||c||c||c|}
\hline Name & Priors & $\ln \left(\mathcal{E} / \mathcal{E}_{\mathrm{HI}}\right)$ & $\mathcal{C}$ & $N$ & $\ln \left(\mathcal{L}_{\max } / \mathcal{E}_{\mathrm{HI}}\right)$ \\
\hline SBI & $\begin{array}{c}\log (\alpha) \in[-5,-2] \\
\log (\beta) \in[-4,-1]\end{array}$ & -0.37 & 1.67 & 4 & 2.29 \\
\hline $\mathrm{SBI}_{\alpha_{\min }}$ & $\begin{array}{c}\alpha=\alpha_{\min } \\
\log (\beta) \in[-4,-1]\end{array}$ & -0.85 & 2.04 & 3 & 2.23 \\
\hline
\end{tabular}

\section{A.41 Spontaneous Symmetry Breaking Inflation (SSBI)}

The SSBI models are described by potentials of the form

$$
V(\phi)=M^{4}\left[1+\alpha\left(\frac{\phi}{M_{\mathrm{Pl}}}\right)^{2}+\beta\left(\frac{\phi}{M_{\mathrm{Pl}}}\right)^{4}\right],
$$

where $\alpha$ and $\beta$ are the two dimensionless parameters. As discussed in Ref. [5], this potential supports six different inflationary regimes according the relative signs of $\alpha$ and $\beta$. They are SSBI1 for $\alpha>0, \beta>0$; SSBI2 for $\alpha<0, \beta<0$; SSBI3 and SSBI4 for $\alpha>0, \beta<0$; SSBI5 and SSBI6 for $\alpha<0, \beta>0$. A priori the parameters $\alpha$ and $\beta$ may take very small values, or not, depending on the underlying theoretical motivations (see Ref. [5]). As a result, we have both considered a Jeffreys and flat prior for those two parameters. There are however some additional restrictions. For SSBI1, inflation ends only for $\alpha>\alpha_{\min }(\beta)$ which fixes an absolute lower limit for the $\alpha$-prior. Moreover, even when this condition is satisfied, SSBI1 is strongly disfavoured when $\alpha$ becomes small and we have only considered $\alpha>\max \left(10^{-3}, \alpha_{\min }\right)$. For SSBI3 and SSBI4, inflation proceeds from the top of the potential, either at increasing field values or at decreasing field values. The shape of the SSBI potential is such that this may occur in a non slow-rolling way with $\epsilon_{2}$ large. These situations violates the slow-roll approximation, and are strongly disfavoured. Therefore, we have added a hard prior rejecting all model parameter values yielding $\epsilon_{2}\left(x_{\text {top }}\right)>0.2, x_{\text {top }}$ being the field value at the top of the potential. Finally, for SSBI5 and SSBI6, there is another value $\alpha_{\max }(\beta)$ above which inflation never ends. As a result, for those scenarios, the prior on $\alpha$ verifies $\alpha<\alpha_{\max }(\beta)$. The following table summarises all the SSBI models considered with the Jeffreys prior choices: 


\begin{tabular}{|c||c||c||c||c||c|}
\hline Name & Priors & $\ln \left(\mathcal{E} / \mathcal{E}_{\mathrm{HI}}\right)$ & $\mathcal{C}$ & $N$ & $\ln \left(\mathcal{L}_{\mathrm{max}} / \mathcal{E}_{\mathrm{HI}}\right)$ \\
\hline SSBI1 & $\begin{array}{c}\max \left[-3, \log \left(\alpha_{\min }\right)\right]<\log (\alpha)<1 \\
\log (\beta) \in[-5,1]\end{array}$ & -4.27 & 2.73 & 4 & -0.08 \\
\hline SSBI2 & $\begin{array}{c}\log (-\alpha) \in[-5,1] \\
\log (-\beta) \in[-5,1]\end{array}$ & -0.54 & 1.74 & 4 & 2.29 \\
\hline SSBI3 & $\begin{array}{c}\log (\alpha) \in\left[\log \left(\alpha_{\min }\right), 1\right] \\
\log (-\beta) \in[-5,1] \\
\epsilon_{2}\left(x_{\mathrm{top}}\right)<0.2\end{array}$ & -2.28 & 4.13 & 4 & 1.83 \\
\hline SSBI4 & $\begin{array}{c}\log (\alpha) \in\left[\log \left(\alpha_{\min }\right), 1\right] \\
\log (-\beta) \in[-5,1] \\
\epsilon_{2}\left(x_{\mathrm{top}}\right)<0.2\end{array}$ & -0.70 & 2.02 & 4 & 2.29 \\
\hline SSBI5 & $\begin{array}{c}\log (-\alpha) \in\left[\log \left(-\alpha_{\max }\right), 1\right] \\
\log (\beta) \in[-5,1]\end{array}$ & -3.02 & 2.40 & 4 & 2.19 \\
\hline SSBI6 & $\begin{array}{c}\log (-\alpha) \in\left[\log \left(-\alpha_{\max }\right), 1\right] \\
\log (\beta) \in[-5,1]\end{array}$ & -3.30 & 3.19 & 4 & 0.72 \\
\hline
\end{tabular}

We have also considered the same models but when the natural values of $\alpha$ and $\beta$ are considered as being $\mathcal{O}(1)$, i.e. with flat priors rather than Jeffreys priors. They are listed in the table below.

\begin{tabular}{|l||c||c||c||c||c|}
\hline Name & Priors & $\ln \left(\mathcal{E} / \mathcal{E}_{\mathrm{HI}}\right)$ & $\mathcal{C}$ & $N$ & $\ln \left(\mathcal{L}_{\max } / \mathcal{E}_{\mathrm{HI}}\right)$ \\
\hline SSBI$_{\mathrm{f}}$ & $\begin{array}{c}\alpha \in\left[\max \left(10^{-3}, \alpha_{\min }\right), 10\right] \\
\beta \in\left[10^{-5}, 10\right]\end{array}$ & -6.24 & 9.08 & 4 & -0.08 \\
\hline SSBI $_{\mathrm{f}}$ & $\begin{array}{c}\alpha \in\left[-10,-10^{-5}\right] \\
\log (-\beta) \in[-5,1]\end{array}$ & -2.65 & 1.99 & 4 & 2.29 \\
\hline SSBI$_{\mathrm{f}}$ & $\begin{array}{c}\alpha \in\left[\alpha_{\min }, 10\right] \\
\beta \in\left[-10,-10^{-5}\right] \\
\epsilon_{2}\left(x_{\mathrm{top}}\right)<0.2\end{array}$ & -2.52 & 2.93 & 4 & 1.83 \\
\hline SSBI$_{\mathrm{f}}$ & $\begin{array}{c}\alpha \in\left[\alpha_{\min }, 10\right] \\
\beta \in\left[-10,-10^{-5}\right] \\
\epsilon_{2}\left(x_{\mathrm{top}}\right)<0.2\end{array}$ & -2.09 & 2.40 & 4 & 2.29 \\
\hline SSBI5 $_{\mathrm{f}}$ & $\begin{array}{c}\alpha \in\left[-10, \alpha_{\max }\right] \\
\beta \in\left[10^{-5}, 10\right]\end{array}$ & -7.11 & 2.93 & 4 & 2.19 \\
\hline SSBI6 $_{\mathrm{f}}$ & $\begin{array}{c}\alpha \in\left[-10, \alpha_{\max }\right] \\
\beta \in\left[10^{-5}, 10\right]\end{array}$ & -6.08 & 10.15 & 4 & 0.71 \\
\hline
\end{tabular}

\section{A.42 Inverse Monomial Inflation (IMI)}

The potential is a extension of the large field inflation potential to negative power indices and read

$$
V(\phi)=M^{4}\left(\frac{\phi}{M_{\mathrm{Pl}}}\right)^{-p}
$$

with $p>0$. Inflation proceeds at increasing field values and ends at the field value $x_{\text {end }}=$ $\phi_{\mathrm{end}} / M_{\mathrm{Pl}}$, which is an additional model parameter. There is however a region, at small field values, which does not support inflation as $\epsilon_{1}(x)>1$. Denoting $x_{\epsilon_{1}=1}$ the solution of $\epsilon_{1}=1$, this implies that $x_{\mathrm{ini}}>x_{\epsilon_{1}=1}$ and there is a minimal acceptable value for $x_{\text {end }}$ such that inflation lasts more than $120 e$-folds. As for the other models, this value $x_{\text {end }}^{\min }$ is numerically determined by solving the field trajectory starting at $x_{\mathrm{ini}}=x_{\epsilon_{1}=1}$ for the specified amount 
of $e$-folds. In the absence of definite constraints on $x_{\mathrm{end}}$, we have chosen a flat prior for $x_{\text {end }} / x_{\text {end }}^{\min } \in[1,100]$ as well as various fixed values of $p=\mathcal{O}(1)$. They are summarised below.

\begin{tabular}{|l||c||c||c||c||c|}
\hline Name & Priors & $\ln \left(\mathcal{E} / \mathcal{E}_{\mathrm{HI}}\right)$ & $\mathcal{C}$ & $N$ & $\ln \left(\mathcal{L}_{\mathrm{max}} / \mathcal{E}_{\mathrm{HI}}\right)$ \\
\hline IMI & $\begin{array}{c}p \in[1,6] \\
x_{\text {end }} / x_{\text {end }}^{\min } \in[1,100]\end{array}$ & -7.79 & 6.69 & 4 & -4.44 \\
\hline IMI1 & $\begin{array}{c}p=1 \\
x_{\text {end }} / x_{\text {end }}^{\min } \in[1,100]\end{array}$ & -7.80 & 0.85 & 3 & -7.37 \\
\hline IMI2 & $\begin{array}{c}p=2 \\
x_{\text {end }} / x_{\text {end }}^{\min } \in[1,100]\end{array}$ & -7.80 & 1.41 & 3 & -7.09 \\
\hline IMI3 & $\begin{array}{c}p=3 \\
x_{\text {end }} / x_{\text {end }}^{\min } \in[1,100]\end{array}$ & -7.80 & 5.88 & 3 & -4.85 \\
\hline IMI4 & $\begin{array}{c}p=4 \\
x_{\text {end }} / x_{\text {end }}^{\min } \in[1,100]\end{array}$ & -7.79 & 6.28 & 3 & -4.65 \\
\hline IMI5 & $\begin{array}{c}p=5 \\
x_{\text {end }} / x_{\text {end }}^{\min } \in[1,100]\end{array}$ & -7.79 & 5.68 & 3 & -4.93 \\
\hline IMI6 & $\begin{array}{c}p=6 \\
x_{\text {end }} / x_{\text {end }}^{\text {min }} \in[1,100]\end{array}$ & -7.78 & 6.63 & 3 & -4.44 \\
\hline
\end{tabular}

\section{A.43 Brane Inflation (BI)}

The potential of brane inflation reads

$$
V_{\mathrm{BI}_{\mathrm{ph}}}(\phi)=M^{4}\left[1-\left(\frac{\phi}{\mu}\right)^{-p}\right],
$$

and depends explicitly on two parameters $\mu$ and $p$. This is an approximated expression derived from KKLMMT-like inflationary scenarios, in which $p=4$ and $\mu \ll M_{\mathrm{Pl}}[74,75]$. In the following, we define $x \equiv \phi / \mu$ and inflation proceeds at decreasing $x$. It is induced by the motion of a brane inside the throat of some compactified extra-dimensions, $\phi$ referring to the position of this brane. Brane inflation can either ends naturally, i.e. when the acceleration of the universe stops, or before if a tachyonic preheating is triggered by brane annihilation. The model has therefore an additional parameter, $x_{\text {stg }}$, which is the field value at which brane annihilation occurs. Denoting by $x_{\epsilon_{1}=1}$ the solution of $\epsilon_{1}(x)=1$, inflation actually ends at the field value $x_{\text {end }}=\max \left(x_{\mathrm{stg}}, x_{\epsilon_{1}=1}\right)$. The parameter $x_{\mathrm{stg}}$ is related to various hidden string parameters such as the flux conserved quantum numbers and the volume of the throat. As shown in Ref. [76], the internal consistency of the model implies that $x_{\text {stg }}>1$, its order of magnitude remaining unknown. Moreover, there is a maximal field value, $\phi_{\mathrm{UV}}$, which corresponds to the brane position at the edge of the throat. As the model only describes brane interactions within the throat, one should impose $\phi<\phi_{\mathrm{UV}}$. As discussed in Ref. [76], the internal consistency of the model imposes that $\phi_{\mathrm{UV}}<2 M_{\mathrm{P} 1}$.

Following these considerations, we have first considered strict priors associated with the string scenario $\left(\mathrm{BI}_{\mathrm{stg}}\right)$, namely $p=4, \log \left(\mu / M_{\mathrm{Pl}}\right) \in[-6, \log (2)], \log \left(x_{\mathrm{stg}}\right) \in[0,3]$ and $\log \left(\phi_{\mathrm{UV}} / M_{\mathrm{Pl}}\right) \in[-2, \log (2)]$. For the sake of generality, we have also considered the nonapproximated potential associated with the KKLMMT model, namely

$$
V_{\mathrm{KKLTI}}(\phi)=\frac{M^{4}}{1+\left(\frac{\phi}{\mu}\right)^{-p}},
$$


under the label $\mathrm{KKLTI}_{\mathrm{stg}}$, and with the same priors as $\mathrm{BI}_{\text {stg. }}$ As one can check in the following table, there is no difference between the two models under those priors.

\begin{tabular}{|l||c||c||c||c||c|}
\hline Name & Priors & $\ln \left(\mathcal{E} / \mathcal{E}_{\mathrm{HI}}\right)$ & $\mathcal{C}$ & $N$ & $\ln \left(\mathcal{L}_{\text {max }} / \mathcal{E}_{\mathrm{HI}}\right)$ \\
\hline \multirow{3}{*}{$\mathrm{BI}_{\mathrm{stg}}$} & $\begin{array}{c}p=4 \\
\log \left(\mu / M_{\mathrm{Pl}}\right) \in[-6, \log (2)]\end{array}$ & -0.33 & 1.91 & 5 & 2.29 \\
& $\begin{array}{c}\log \left(x_{\mathrm{stg}}\right) \in[0,3] \\
\log \left(\phi_{\mathrm{UV}} / M_{\mathrm{Pl}}\right) \in[-2, \log (2)]\end{array}$ & & & & \\
\hline & $\begin{array}{c}p=4 \\
\log \left(\mu / M_{\mathrm{Pl}}\right) \in[-6, \log (2)] \\
\operatorname{KKLTI}\end{array}$ & -0.32 & 1.92 & 5 & 2.29 \\
& $\begin{array}{c}\log \left(x_{\mathrm{stg}}\right) \in[0,3] \\
\log \left(\phi_{\mathrm{UV}} / M_{\mathrm{Pl}}\right) \in[-2, \log (2)]\end{array}$ & & & & \\
\hline
\end{tabular}

Then, we have allowed for other phenomenological scenarios that would be based on the same potentials by relaxing $p$ and allowing $\mu$ to become super-Planckian. Out of the string framework, there is no motivation to keep $x_{\mathrm{stg}}$ and $\phi_{\mathrm{UV}}$ as extra model parameters and we have instead assumed that inflation ends at $x_{\text {end }}=x_{\epsilon_{1}=1}$. However, for $\mu>M_{\mathrm{Pl}}$, inflation within the potential (A.80) or (A.81) may yield different observable predictions. As a result, we have separated the models in which $\mu<M_{\mathrm{P} 1}$ from those in which $\mu$ can take any values. The phenomenological models considered, and their priors, are enumerated in the following table.

\begin{tabular}{|l||c||c||c||c||c|}
\hline Name & Priors & $\ln \left(\mathcal{E} / \mathcal{E}_{\mathrm{HI}}\right)$ & $\mathcal{C}$ & $N$ & $\ln \left(\mathcal{L}_{\max } / \mathcal{E}_{\mathrm{HI}}\right)$ \\
\hline KKLTI & $\begin{array}{c}p \in[2,10] \\
\log \left(\mu / M_{\mathrm{Pl}}\right) \in[-3,3]\end{array}$ & -0.34 & 1.90 & 4 & 2.29 \\
\hline $\mathrm{KKLTI}_{\mathrm{s}}$ & $\begin{array}{c}p \in[2,10] \\
\log \left(\mu / M_{\mathrm{Pl}}\right) \in[-3,0]\end{array}$ & 0.02 & 1.85 & 4 & 2.29 \\
\hline $\mathrm{BI}_{\mathrm{ph}}$ & $\begin{array}{c}p \in[2,10] \\
\log \left(\mu / M_{\mathrm{Pl}}\right) \in[-3,3]\end{array}$ & -0.19 & 2.14 & 4 & 2.29 \\
\hline $\mathrm{BI}_{\mathrm{s}}$ & $\begin{array}{c}p \in[2,10] \\
\log \left(\mu / M_{\mathrm{Pl}}\right) \in[-3,0]\end{array}$ & 0.03 & 1.84 & 4 & 2.29 \\
\hline $\mathrm{BI}_{1 \mathrm{~s}}$ & $\begin{array}{c}p=1 \\
\log \left(\mu / M_{\mathrm{Pl}}\right) \in[-3,0]\end{array}$ & -0.21 & 2.19 & 3 & 2.29 \\
\hline $\mathrm{BI}_{2 \mathrm{~s}}$ & $\begin{array}{c}p=2 \\
\log \left(\mu / M_{\mathrm{Pl}}\right) \in[-3,0]\end{array}$ & -0.08 & 2.05 & 3 & 2.29 \\
\hline $\mathrm{BI}_{3 \mathrm{~s}}$ & $\begin{array}{c}p=3 \\
\log \left(\mu / M_{\mathrm{Pl}}\right) \in[-3,0]\end{array}$ & -0.02 & 1.96 & 3 & 2.29 \\
\hline $\mathrm{BI}_{4 \mathrm{~s}}$ & $\begin{array}{c}p=4 \\
\log \left(\mu / M_{\mathrm{Pl}}\right) \in[-3,0]\end{array}$ & 0.01 & 1.91 & 3 & 2.29 \\
\hline $\mathrm{BI}_{5 \mathrm{~s}}$ & $\begin{array}{c}p=5 \\
\log \left(\mu / M_{\mathrm{Pl}}\right) \in[-3,0]\end{array}$ & 0.02 & 1.87 & 3 & 2.29 \\
\hline $\mathrm{BI}_{6 \mathrm{~s}}$ & $\begin{array}{c}p=6 \\
\log \left(\mu / M_{\mathrm{Pl}}\right) \in[-3,0]\end{array}$ & 0.04 & 1.82 & 3 & 2.29 \\
\hline
\end{tabular}

\section{A.44 Running-mass Inflation (RMI)}

The running-mass inflationary models, denoted RMI, have a potential of the form

$$
V(\phi)=M^{4}\left[1-\frac{c}{2}\left(-\frac{1}{2}+\ln \frac{\phi}{\phi_{0}}\right) \frac{\phi^{2}}{M_{\mathrm{Pl}}^{2}}\right],
$$


which supports four different inflationary regimes, namely RMI1 $\left(\phi<\phi_{0}, c>0\right)$, RMI2 $\left(\phi>\phi_{0}, c>0\right)$, RMI3 $\left(\phi<\phi_{0}, c<0\right)$ and RMI4 $\left(\phi>\phi_{0}, c<0\right)$, see Ref. [5]. In addition to the constant $c$ and the vev $\phi_{0}$, the field value $\phi_{\text {end }}$ at which inflation ends has to be specified making RMI a three-parameters model. The model describing loop corrections over a polynomial expansion, the constant $c$ cannot be too small and the vev $\phi_{0}$ must be sub-Planckian. The order of magnitude of $\phi_{0}$ being unspecified, we have chosen a Jeffreys prior in $\log \left(\phi_{0} / M_{\mathrm{Pl}}\right) \in[-2,0]$. For RMI1, RMI2 and RMI3, the likelihood has a flat direction along the parameter $\phi_{0}$ such that the evidence is independent of the lower bound on $\phi_{0}$. For RMI4, the likelihood is vanishing when $\phi_{0}$ becomes small, and the evidence is accordingly decreased if the prior lower bound on $\log \left(\phi_{0} / M_{\mathrm{Pl}}\right)$ is pushed to smaller values.

As discussed in Ref. [5], within supersymmetry, natural values of $c \simeq 10^{-2}$ to $10^{-1}$ for soft masses values matching the energy scale of inflation. This suggest to take a flat prior for $c$ encompassing those values. For other type of couplings, $c$ may take smaller values and we therefore consider another motivated prior in which the order of magnitude of $c$ is unknown, e.g. $\log (c) \in[-3,-1]$.

Finally, the field value $\phi_{\text {end }}$ is constrained to be in the inflationary region of interest. The shape of the potential therefore gives the natural prior bounds for $x_{\text {end }} \equiv \phi_{\text {end }} / \phi_{0}$, i.e. $x_{\text {end }} \in[1 / e, 1]$ for RMI1 and RMI3, $x_{\text {end }} \in[1, e]$ for RMI2. For RMI4, one still has $x_{\text {end }}>1$ but choosing the prior upper limit requires some precaution. Indeed, the potential is an increasing function of $\phi$, which approaches large field inflation asymptotically, and inflation proceeds at decreasing field values, bounded from below by $x_{\text {end }}$. Since the large field regime is not acceptable for RMI4, one has to require the initial field value $x_{\text {ini }}<x_{\epsilon_{1}^{\max }}$. Here $x_{\epsilon_{1}^{\max }}$ is the field value at which the first Hubble flow function is maximal, which is the frontier between the vacuum dominated regime and the large field one. As for the other models, adding the "hard prior" that inflation lasts longer than $120 e$-folds ensures the existence of a maximal value $x_{\text {end }}^{\max }$, which is obtained by integrating the field trajectory from $x_{\text {ini }}=x_{\epsilon_{1}^{\max }}$. This is a complicated functions of the other parameters which is only known numerically. The parameter space of RMI 4 is therefore sampled with a flat prior for $x_{\text {end }} \in\left[1, x_{\text {end }}^{\max }\right]$.

Finally, for RMI1 and RMI2, we have added another "hard prior" to avoid an infinite number of $e$-folds to occur at the top of the potential by requiring $\epsilon_{1}\left(x_{\text {ini }}\right)$ to be larger than the numerical machine precision. This has no observable consequences as the parameter space volume cut remains extremely small and those cases would correspond otherwise to $n_{\mathrm{S}}=1$ and are disfavoured. All these considerations are summarised in the following table. 


\begin{tabular}{|c|c|c|c|c|c|}
\hline Name & Priors & $\ln \left(\mathcal{E} / \mathcal{E}_{\mathrm{HI}}\right)$ & $\mathcal{C}$ & $N$ & $\ln \left(\mathcal{L}_{\max } / \mathcal{E}_{\mathrm{HI}}\right)$ \\
\hline $\mathrm{RMI}_{1}$ & $\begin{array}{c}c \in[0.01,0.2] \\
\log \left(\phi_{0} / M_{\mathrm{Pl}}\right) \in[-2,0] \\
x_{\mathrm{end}} \in[1 / e, 1]\end{array}$ & -2.03 & 1.94 & 5 & 2.29 \\
\hline $\mathrm{RMI}_{11}$ & $\begin{array}{c}\log (c) \in[-3,-1] \\
\log \left(\phi_{0} / M_{\mathrm{Pl}}\right) \in[-2,0] \\
x_{\text {end }} \in[1 / e, 1]\end{array}$ & -1.41 & 2.04 & 5 & 2.29 \\
\hline $\mathrm{RMI}_{2}$ & $\begin{array}{c}c c \in[0.01,0.2] \\
\log \left(\phi_{0} / M_{\mathrm{Pl}}\right) \in[-2,0] \\
x_{\text {end }} \in[1, e]\end{array}$ & -2.18 & 1.80 & 5 & 2.29 \\
\hline $\mathrm{RMI}_{21}$ & $\begin{array}{c}\log (c) \in[-3,-1] \\
\log \left(\phi_{0} / M_{\mathrm{Pl}}\right) \in[-2,0] \\
x_{\text {end }} \in[1, e]\end{array}$ & -1.14 & 2.04 & 5 & 2.29 \\
\hline $\mathrm{RMI}_{3}$ & $\begin{array}{c}c \in[-0.2,-0.01] \\
\log \left(\phi_{0} / M_{\mathrm{Pl}}\right) \in[-2,0] \\
x_{\text {end }} \in[1 / e, 1]\end{array}$ & -1.96 & 2.05 & 5 & 2.29 \\
\hline $\mathrm{RMI}_{31}$ & $\begin{array}{c}\log (-c) \in[-3,-1] \\
\log \left(\phi_{0} / M_{\mathrm{Pl}}\right) \in[-2,0] \\
x_{\text {end }} \in[1 / e, 1]\end{array}$ & -2.84 & 2.16 & 5 & 2.29 \\
\hline $\mathrm{RMI}_{4}$ & $\begin{array}{c}c \in[-0.2,-0.01] \\
\log \left(\phi_{0} / M_{\mathrm{Pl}}\right) \in[-2,0] \\
x_{\text {end }} \in\left[1, x_{\text {end }}^{\max }\right]\end{array}$ & -25.90 & 9.54 & 5 & -13.85 \\
\hline $\mathrm{RMI}_{41}$ & $\begin{array}{c}\log (-c) \in[-3,-1] \\
\log \left(\phi_{0} / M_{\mathrm{Pl}}\right) \in[-2,0] \\
x_{\text {end }} \in\left[1, x_{\text {end }}^{\max }\right]\end{array}$ & -9.26 & 1.28 & 5 & -7.13 \\
\hline
\end{tabular}

\section{A.45 Valley Hybrid Inflation (VHI)}

The potential is parametrised by two parameters $p$ and $\mu$ and reads

$$
V(\phi)=M^{4}\left[1+\left(\frac{\phi}{\mu}\right)^{p}\right]
$$

$p>0$ being the power index and $\mu$ is a typical vev. Because this expression only describes inflation along the valley of the genuine two-field hybrid inflationary scenario, the vev $\mu$ is forced to be super-Planckian. As discussed in Refs. [77-81], this condition is required to get enough $e$-folds of inflation occurring in the valley. Another implicit prior is to assume that the parameters associated with the other field are such that the regime of waterfall inflation does not take place. As discussed in Ref. [5], the dynamics of VHI is significantly different if $p>1$ or $p<1$ and the two classes are considered. In addition to $\mu$ and $p$, hybrid inflation ends by tachyonic instability and the field value $x_{\text {end }} \equiv \phi_{\text {end }} / \mu$ at which this occurs is an extra model parameter. As for RMI, our prior is to restrain VHI to the vacuum dominated regime only, i.e. $x_{\text {ini }}<x_{\epsilon_{1}^{\max }}$ where $x_{\epsilon_{1}^{\max }}$ is the frontier between the vacuum dominated regime and the large field one. From this limit, requiring inflation to support at least $120 e$-folds gives a numerical upper bound $x_{\text {end }}<x_{\text {end }}^{\max }$. The quantity $x_{\text {end }}^{\max }$ is determined numerically using

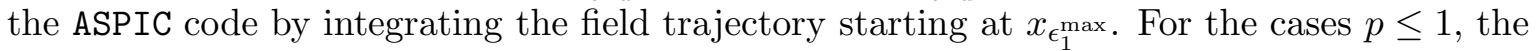
VHI potential does not support inflation around $x=0$ as $\epsilon_{1}$ diverges in this limit. For those, we therefore consider a prior $x_{\text {end }}>x_{\text {end }}^{\min }$ where $x_{\text {end }}^{\text {min }}=x_{\epsilon_{1}=1}^{-}$is the solution of $\epsilon_{1}=1$ in the vacuum dominated region. For $p>1$, the tachyonic instability can take place at arbitrarily 
small field values and $x_{\text {end }}^{\min }=0$ (up to machine precision limitations). Notice that the upper bounds of the $p$ and $\mu$ priors have been fixed to arbitrary values. All the models considered for the VHI scenarios are listed below and are all ruled out, independently of their priors.

\begin{tabular}{|c|c|c|c|c|c|}
\hline Name & Priors & $\ln \left(\mathcal{E} / \mathcal{E}_{\mathrm{HI}}\right)$ & $\mathcal{C}$ & $N$ & $\ln \left(\mathcal{L}_{\max } / \mathcal{E}_{\mathrm{HI}}\right)$ \\
\hline VHI & $\begin{array}{c}p \in] 1,6] \\
\log \left(\mu / M_{\mathrm{Pl}}\right) \in[0,3] \\
x_{\mathrm{end}} \in\left[x_{\mathrm{end}}^{\min }, x_{\mathrm{end}}^{\max }\right]\end{array}$ & -7.81 & 16.46 & 5 & 0.43 \\
\hline $\mathrm{VHI}_{p<1}$ & $\begin{array}{c}p \in[0,0.9] \\
\log \left(\mu / M_{\mathrm{Pl}}\right) \in[0,3] \\
x_{\mathrm{end}} \in\left[x_{\mathrm{end}}^{\min }, x_{\mathrm{end}}^{\max }\right]\end{array}$ & -7.80 & 20.09 & 5 & 2.27 \\
\hline $\mathrm{VHI}_{1 / 2}$ & $\begin{array}{c}p=1 / 2 \\
\log \left(\mu / M_{\mathrm{Pl}}\right) \in[0,3] \\
x_{\text {end }} \in\left[x_{\text {end }}^{\text {min }}, x_{\text {end }}^{\text {max }}\right]\end{array}$ & -7.80 & 19.57 & 4 & 1.99 \\
\hline $\mathrm{VHI}_{1}$ & $\begin{array}{c}p=1 \\
\log \left(\mu / M_{\mathrm{Pl}}\right) \in[0,3] \\
x_{\text {end }} \in\left[x_{\text {end }}^{\text {min }}, x_{\text {end }}^{\text {max }}\right]\end{array}$ & -7.80 & 17.14 & 4 & 0.79 \\
\hline $\mathrm{VHI}_{2}$ & $\begin{array}{c}p=2 \\
\log \left(\mu / M_{\mathrm{Pl}}\right) \in[0,3] \\
x_{\text {end }} \in\left[x_{\text {end }}^{\min }, x_{\text {end }}^{\max }\right]\end{array}$ & -7.80 & 15.42 & 4 & -0.09 \\
\hline $\mathrm{VHI}_{3}$ & $\begin{array}{c}p=3 \\
\log \left(\mu / M_{\mathrm{Pl}}\right) \in[0,3] \\
x_{\mathrm{end}} \in\left[x_{\mathrm{end}}^{\min }, x_{\mathrm{end}}^{\max }\right]\end{array}$ & -7.81 & 14.66 & 4 & -0.47 \\
\hline $\mathrm{VHI}_{4}$ & $\begin{array}{c}p=4 \\
\log \left(\mu / M_{\mathrm{Pl}}\right) \in[0,3] \\
x_{\mathrm{end}} \in\left[x_{\mathrm{end}}^{\min }, x_{\mathrm{end}}^{\max }\right]\end{array}$ & -7.82 & 14.15 & 4 & -0.72 \\
\hline
\end{tabular}

\section{A.46 Dynamical Supersymmetric Inflation (DSI)}

The potential is an extension of the VHI one to negative power index and reads

$$
V(\phi)=M^{4}\left[1+\left(\frac{\phi}{\mu}\right)^{-p}\right],
$$

while this class of model naturally appears in supersymmetric theories (see Ref. [5]). As such, the vev $\mu$ should be always sub-Planckian. For the priors, we have either considered the typical values of Refs. [82, 83], i.e. a flat prior for $\mu$ around $10^{-7}$ (model DSI on , which is also relaxed to allow for any other phenomenological models of the same kind (DSI). Inflation takes place at increasing field value and the end of inflation $x_{\text {end }}=\phi_{\text {end }} / \mu$ is an additional parameter. Moreover, as discussed in Ref. [5], inflation can only take place in the region $x>x_{\epsilon_{1}=1}$, where $x_{\epsilon_{1}=1}$ is the solution of $\epsilon_{1}=1$. This provides a lower bound for $x_{\mathrm{ini}}$, and therefore, complemented with our hard prior that inflation lasts more than $120 e$-folds, this gives $x_{\text {end }}>x_{\text {end }}^{\min }$. As for VHI, the quantity $x_{\text {end }}^{\min }$ has to be numerically evaluated by integrating the field trajectory over $120 e$-folds starting at $x_{\text {ini }}=x_{\epsilon_{1}=1}$. Moreover, within the supersymmetric framework in which this potential is derived, there are additional terms lifting $V(\phi)$ at large field values which can be ignored provided $\phi$ is not too large. This gives a natural upper bound for the prior on $x_{\text {end }}$. More specifically, these terms are of the form 
$\Delta V=\phi^{q+4} / M_{\mathrm{Pl}}^{q}$ such that the corrected potential exhibits a global minimum at a field value $x_{V_{\text {min }}}$. Requiring $x_{\text {end }} \ll x_{V^{\min }}$ gives the absolute upper bound

$$
x_{\text {end }} \ll x_{\text {end }}^{\max } \equiv\left[43200 \pi^{2} \frac{p^{3}}{q+4} P_{*}\left(\frac{M_{\mathrm{P} 1}}{\mu}\right)^{q+6}\right]^{1 /(3 p+q+6)} .
$$

As a motivated case, we have chosen $q=8$ and a Jeffreys' prior on $x_{\text {end }}$ in $\left[x_{\text {end }}^{\min }, x_{\text {end }}^{\max }\right]$. The case $p=2$ has been considered as an independent model as it corresponds to the socalled inverse mutated scenarios. In summary, the following models and priors have been considered:

\begin{tabular}{|c|c|c|c|c|c|}
\hline Name & Priors & $\ln \left(\mathcal{E} / \mathcal{E}_{\mathrm{HI}}\right)$ & $\overline{\mathcal{C}}$ & $N$ & $\ln \left(\mathcal{L}_{\max } / \mathcal{E}_{\mathrm{HI}}\right)$ \\
\hline DSI & $\begin{array}{c}p \in] 1,6] \\
\log \left(\mu / M_{\mathrm{Pl}}\right) \in[-5,0] \\
\log \left(\frac{x_{\text {end }}-x_{\text {end }}^{\min }}{x_{\text {end }}^{\max }-x_{\text {end }}^{\min }}\right) \in[-5,-0.7]\end{array}$ & -8.51 & 0.07 & 5 & -7.71 \\
\hline $\mathrm{DSI}_{\mathrm{o}}$ & $\begin{array}{c}p \in] 1,6] \\
\mu / M_{\mathrm{P} 1} \in\left[10^{-9}, 10^{-6}\right] \\
\log \left(\frac{x_{\mathrm{end}}-x_{\mathrm{end}}^{\min }}{x_{\mathrm{end}}^{\max }-x_{\mathrm{end}}^{\min }}\right) \in[-5,-0.7]\end{array}$ & -8.47 & 0.07 & 5 & -7.71 \\
\hline $\mathrm{DSI}_{2}$ & $\begin{array}{c}p=2 \\
\mu / M_{\mathrm{P} 1} \in\left[10^{-9}, 10^{-6}\right] \\
\log \left(\frac{x_{\mathrm{end}}-x_{\mathrm{end}}^{\min }}{x_{\mathrm{end}}^{\max }-x_{\mathrm{end}}^{\min }}\right) \in[-5,-0.7]\end{array}$ & -8.40 & 0.07 & 4 & -7.71 \\
\hline
\end{tabular}

\section{A.47 Generalised Mixed Large Field Inflation (GMLFI)}

The potential mixes two large field monomials and reads

$$
V(\phi)=M^{4}\left(\frac{\phi}{M_{\mathrm{Pl}}}\right)^{p}\left[1+\alpha\left(\frac{\phi}{M_{\mathrm{Pl}}}\right)^{q}\right],
$$

where $p$ and $q$ are power indices and $\alpha$ a constant. The model has three parameters and their priors have been chosen on phenomenological grounds. In particular, because GMLFI allows to discuss the effects stemming from combining together two LFI models, it motivates to fix $p$ and $q$ to all the possible theoretically motivated combination of pure LFI models. One can also view GMLFI as a new class of models and let $p, q$ and $\alpha$ freely varying. A priori, the parameter $\alpha$ can be very small such that it should be sampled along a Jeffreys prior. We have considered the following cases: 


\begin{tabular}{|c|c|c|c|c|c|}
\hline Name & Priors & $\ln \left(\mathcal{E} / \mathcal{E}_{\mathrm{HI}}\right)$ & $\mathcal{C}$ & $N$ & $\ln \left(\mathcal{L}_{\max } / \mathcal{E}_{\mathrm{HI}}\right)$ \\
\hline GMLFI & $\begin{array}{c}p \in[1,6] \\
\log (\alpha) \in[-5,1] \\
q \in[1,6]\end{array}$ & -5.15 & 3.90 & 5 & 0.79 \\
\hline $\mathrm{GMLFI}_{2 / 3,1 / 3}$ & $\begin{array}{c}p=2 / 3 \\
\log (\alpha) \in[-5,1] \\
q=1 / 3\end{array}$ & -1.27 & 2.31 & 3 & 1.24 \\
\hline $\mathrm{GMLFI}_{2 / 3,4 / 3}$ & $\begin{array}{c}p=2 / 3 \\
\log (\alpha) \in[-5,1] \\
q=4 / 3\end{array}$ & -1.69 & 2.74 & 3 & 1.24 \\
\hline GMLFI $_{1,1}$ & $\begin{aligned} p & =1 \\
\log (\alpha) & \in[-5,1] \\
q & =1\end{aligned}$ & -1.86 & 2.35 & 3 & 0.79 \\
\hline GMLFI $_{1,2}$ & $\begin{aligned} p & =1 \\
\log (\alpha) & \in[-5,1] \\
q & =2\end{aligned}$ & -2.52 & 2.69 & 3 & 0.79 \\
\hline $\mathrm{GMLFI}_{1,3}$ & $\begin{aligned} p & =1 \\
\log (\alpha) & \in[-5,1] \\
q & =3\end{aligned}$ & -3.64 & 3.13 & 3 & 0.63 \\
\hline $\mathrm{GMLFI}_{2,1}$ & $\begin{aligned} p & =2 \\
\log (\alpha) & \in[-5,1] \\
q & =1\end{aligned}$ & -3.05 & 2.08 & 3 & -0.08 \\
\hline MLFI & $\begin{aligned} p & =2 \\
\log (\alpha) & \in[-5,1] \\
q & =2\end{aligned}$ & -3.77 & 2.39 & 3 & -0.09 \\
\hline $\mathrm{GMLFI}_{2,3}$ & $\begin{aligned} p & =2 \\
\log (\alpha) & \in[-5,1] \\
q & =3\end{aligned}$ & -5.23 & 3.47 & 3 & -0.39 \\
\hline $\mathrm{GMLFI}_{3,1}$ & $\begin{aligned} p & =3 \\
\log (\alpha) & \in[-5,1] \\
q & =1\end{aligned}$ & -4.83 & 3.29 & 3 & -1.02 \\
\hline $\mathrm{GMLFI}_{3,2}$ & $\begin{aligned} p & =3 \\
\log (\alpha) & \in[-5,1] \\
q & =2\end{aligned}$ & -5.60 & 4.06 & 3 & -1.04 \\
\hline $\mathrm{GMLFI}_{3,3}$ & $\begin{aligned} p & =3 \\
\log (\alpha) & \in[-5,1] \\
q & =3\end{aligned}$ & -7.43 & 6.87 & 3 & -1.90 \\
\hline
\end{tabular}

Notice that the case $p=2, q=2$ is also referred to as MLFI in Ref. [5].

\section{A.48 Logarithmic Potential Inflation (LPI)}

These scenarios are parametrised by a potential of the form

$$
V(\phi)=M^{4}\left(\frac{\phi}{\phi_{0}}\right)^{p}\left(\ln \frac{\phi}{\phi_{0}}\right)^{q} .
$$

Some specific combinations of $p$ and $q$ match various Yang-Mills composite models LPI1 $1_{4,1}$ $(p=4, q=1), \mathrm{LPI}_{4,2}(p=1, q=2)$ and $\mathrm{LPI}_{4,3}(p=4, q=3)[73,84]$. Others 
combinations are phenomenological [85]. Because the potential admits a local maximum at $x=x_{V_{\max }}$, with $x=\phi / \phi_{0}$, inflation can take place in three domains: LPI1 for $x>1$, LPI2 for $x_{V_{\max }}<x<1$ and LPI3 for $x<x_{V_{\max }}$. Let us notice that for both LPI2 and LPI3, the potential is well-defined only if $q$ is an even integer. For LPI1, both $p$ and $q$ can take arbitrary real values. The vev $\phi_{0}$ is not constrained for LPI1, and we have chosen a Jeffreys prior encompassing both sub-Planckian and super-Planckian values. On the contrary, in the LPI1 and LPI2 domains, $\phi_{0}$ must be deeply super-Planckian to allow for slow-rolling inflation. The models and priors considered are listed below.

\begin{tabular}{|c|c|c|c|c|c|}
\hline Name & Priors & $\ln \left(\mathcal{E} / \mathcal{E}_{\mathrm{HI}}\right)$ & $\overline{\mathcal{C}}$ & $N$ & $\ln \left(\mathcal{L}_{\max } / \mathcal{E}_{\mathrm{HI}}\right)$ \\
\hline LPI1 & $\begin{array}{c}p \in[1,6] \\
\log \left(\phi_{0} / M_{\mathrm{P} 1}\right) \in[-3,3] \\
q \in[1,6]\end{array}$ & -3.95 & 3.84 & 5 & 0.77 \\
\hline $\mathrm{LPI}_{4,1}$ & $\begin{array}{c}p=4 \\
\log \left(\phi_{0} / M_{\mathrm{Pl}}\right) \in[-3,3] \\
q=1\end{array}$ & -3.27 & 2.79 & 3 & 0.69 \\
\hline $\mathrm{LPI}_{4,2}$ & $\begin{array}{c}p=4 \\
\log \left(\phi_{0} / M_{\mathrm{Pl}}\right) \in[-3,3] \\
q=2\end{array}$ & -4.42 & 2.90 & 3 & -0.14 \\
\hline $\mathrm{LPI}_{4,3}$ & $\begin{array}{c}p=4 \\
\log \left(\phi_{0} / M_{\mathrm{Pl}}\right) \in[-3,3] \\
q=3\end{array}$ & -5.99 & 5.28 & 3 & -1.14 \\
\hline $\mathrm{LPI}_{2}$ & $\begin{array}{c}p \in[1,6] \\
\log \left(\phi_{0} / M_{\mathrm{Pl}}\right) \in[2,5] \\
q=2\end{array}$ & -2.47 & 3.82 & 4 & 1.14 \\
\hline $\mathrm{LPI}_{4}$ & $\begin{array}{c}p \in[1,6] \\
\log \left(\phi_{0} / M_{\mathrm{Pl}}\right) \in[2,5] \\
q=4\end{array}$ & -5.97 & 7.85 & 4 & -0.18 \\
\hline $\mathrm{LPI}_{6}$ & $\begin{array}{c}p \in[1,6] \\
\log \left(\phi_{0} / M_{\mathrm{Pl}}\right) \in[2,5] \\
q=6\end{array}$ & -8.15 & 7.64 & 4 & -3.24 \\
\hline $\mathrm{LPI}_{2}$ & $\begin{array}{c}p \in[1,6] \\
\log \left(\phi_{0} / M_{\mathrm{Pl}}\right) \in[2,5] \\
q=2\end{array}$ & -2.67 & 4.58 & 4 & 1.98 \\
\hline $\mathrm{LPI}_{4}$ & $\begin{array}{c}p \in[1,6] \\
\log \left(\phi_{0} / M_{\mathrm{Pl}}\right) \in[2,5] \\
q=4 \\
\end{array}$ & -2.04 & 4.03 & 4 & 2.26 \\
\hline $\mathrm{LPI}_{6}$ & $\begin{array}{c}p \in[1,6] \\
\log \left(\phi_{0} / M_{\mathrm{Pl}}\right) \in[2,5] \\
q=6\end{array}$ & -1.71 & 3.56 & 4 & 2.28 \\
\hline
\end{tabular}

\section{A.49 Constant $n_{\mathrm{S}}$ D Inflation (CNDI)}

The potential has two parameters $\alpha, \beta$ and reads

$$
V(\phi)=\frac{M^{4}}{\left[1+\beta \cos \left(\alpha \frac{\phi}{M_{\mathrm{Pl}}}\right)\right]^{2}} .
$$


As discussed in Ref. [5], the only regime of cosmological interest has $x=\phi / M_{\mathrm{Pl}}$ small and $\beta>1$. In that situation, the field value at which inflation ends should be specified, namely $x_{\text {end }}=\phi_{\text {end }} / M_{\mathrm{Pl}}$. Moreover, if $x$ becomes too large, inflation cannot even start because there exists a "forbidden" range of field values in which $\epsilon_{1}(x)>1$. As a result, there is a maximal value for $x_{\mathrm{ini}}=x_{\epsilon_{1}=1}^{-}, x_{\epsilon_{1}=1}^{-}$being the smallest root of the equation $\epsilon_{1}=1$. As for the other models, by imposing to get at least $120 \mathrm{e}$-folds of inflation, the maximal values of $x_{\text {ini }}$ translates into a maximal value $x_{\text {end }}^{\max }$ thereby constituting the upper bound of the $x_{\text {end }}$ 's prior. Concerning the parameter $\alpha$, the genuine CNDI model is designed to produce a constant spectral index and, as discussed in Ref. [5], this occurs for not too small, neither not too large values of $\alpha$. The priors chosen are summarised in the following table.

\begin{tabular}{|c||c||c||c||c||c|}
\hline Name & Priors & $\ln \left(\mathcal{E} / \mathcal{E}_{\mathrm{HI}}\right)$ & $\mathcal{C}$ & $N$ & $\ln \left(\mathcal{L}_{\max } / \mathcal{E}_{\mathrm{HI}}\right)$ \\
\hline \multirow{2}{*}{ CNDI } & $\beta \in[1.1,6]$ & & & & \\
& $\begin{array}{c}\log (\alpha) \in[-2,-1] \\
x_{\text {end }} \in\left[0, x_{\text {end }}^{\text {max }}\right]\end{array}$ & -7.91 & 6.41 & 5 & -4.55 \\
\hline
\end{tabular}

\section{References}

[1] Planck Collaboration Collaboration, P. Ade et al., Planck 2013 results. XVI. Cosmological parameters, arXiv: 1303.5076.

[2] R. Trotta, Forecasting the Bayes factor of a future observation, Mon.Not.Roy.Astron.Soc. 378 (2007) 819-824, [astro-ph/0703063].

[3] Planck Collaboration Collaboration, P. Ade et al., Planck 2013 results. XXII. Constraints on inflation, arXiv: 1303.5082.

[4] Planck Collaboration Collaboration, P. Ade et al., Planck 2013 Results. XXIV. Constraints on primordial non-Gaussianity, arXiv:1303.5084.

[5] J. Martin, C. Ringeval, and V. Vennin, Encyclopaedia Inflationaris, arXiv:1303.3787.

[6] C. Ringeval, Fast Bayesian inference for slow-roll inflation, arXiv:1312.2347.

[7] R. T. Cox, Probability, Frequency and Reasonable Expectation, American Journal of Physics 14 (Jan., 1946) 1-13.

[8] H. Jeffreys, Theory of probability, Oxford University Press, Oxford Classics series (reprinted 1998) (1961).

[9] B. de Finetti, Theory of probability. John Wiley \& Sons, Chichester, UK, 1974. reprinted 1995.

[10] J. M. Bernardo and A. F. M. Smith, Bayesian Theory. John Wiley \& Sons, Chicester, UK, 1994.

[11] G. E. P. Box and G. C. Tiao, Bayesian Inference in Statistical Analysis. John Wiley \& Sons, Chicester, UK, 1992.

[12] E. T. Jaynes, Probability Theory. The logic of science. Cambridge University Press, Cambridge, UK, 2003.

[13] J. Berger, Could fisher, jeffreys and neyman have agreed on testing?, Statistical Science 18 (2003), no. 1 1-12. rejoinder: ibid., 28-32.

[14] R. Trotta, Applications of Bayesian model selection to cosmological parameters, Mon. Not. Roy. Astron. Soc. 378 (2007) 72-82, [astro-ph/0504022].

[15] R. Trotta, Bayes in the sky: Bayesian inference and model selection in cosmology, Contemp. Phys. 49 (2008) 71-104, [arXiv:0803.4089]. 
[16] C. Gordon and R. Trotta, Bayesian Calibrated Significance Levels Applied to the Spectral Tilt and Hemispherical Asymmetry, Mon. Not. Roy. Astron. Soc. 382 (2007) 1859-1863, [arXiv:0706.3014].

[17] M. March, G. Starkman, R. Trotta, and P. Vaudrevange, Should we doubt the cosmological constant?, Mon.Not.Roy.Astron.Soc. 410 (2011) 2488-2496, [arXiv:1005.3655].

[18] J. O. Berger and T. Sellke, Testing a Point Null Hypothesis: The Irreconcilability of P Values and Evidence, J. Am. Stat. Ass 82 (1987) 112-122.

[19] J. O. Berger and M. Bayarri, The interplay of Bayesian and Frequentist analysis, Stat. Science 19 (2004) 58-80.

[20] V. E. Johnson, Revised standards for statistical evidence, PNAS (2013) Published online before print: doi: 10.1073/pnas.1313476110.

[21] R. D. Cousins, The Jeffreys-Lindley Paradox and Discovery Criteria in High Energy Physics, arXiv: 1310.3791.

[22] S. Kullback and R. Leibler, , Ann. Math. Stat. 22 (1951) 79-86.

[23] M. Kunz, R. Trotta, and D. Parkinson, Measuring the effective complexity of cosmological models, Phys. Rev. D74 (2006) 023503, [astro-ph/0602378].

[24] D. Spiegelhalter et al., Bayesian Measures of Model Complexity and Fit, J. Roy. Stat. Soc. B 64 (2002) 583-639.

[25] F. Feroz, K. Cranmer, M. Hobson, R. Ruiz de Austri, and R. Trotta, Challenges of Profile Likelihood Evaluation in Multi-Dimensional SUSY Scans, JHEP 1106 (2011) 042, [arXiv: 1101.3296$]$.

[26] F. Feroz and M. P. Hobson, Multimodal nested sampling: an efficient and robust alternative to Markov Chain Monte Carlo methods for astronomical data analyses, Mon. Not. R. Astron. Soc. 384 (Feb., 2008) 449-463, [arXiv:0704.3704].

[27] F. Feroz, M. P. Hobson, and M. Bridges, MULTINEST: an efficient and robust Bayesian inference tool for cosmology and particle physics, Mon. Not. R. Astron. Soc. 398 (Oct., 2009) 1601-1614, [arXiv:0809.3437].

[28] J. Martin and C. Ringeval, Inflation after WMAP3: Confronting the slow-roll and exact power spectra to CMB data, JCAP 0608 (2006) 009, [astro-ph/0605367].

[29] J. Martin and C. Ringeval, First CMB Constraints on the Inflationary Reheating Temperature, Phys. Rev. D82 (2010) 023511, [arXiv:1004.5525].

[30] J. Martin, C. Ringeval, and R. Trotta, Hunting Down the Best Model of Inflation with Bayesian Evidence, Phys.Rev. D83 (2011) 063524, [arXiv:1009.4157].

[31] R. Easther and H. V. Peiris, Bayesian Analysis of Inflation II: Model Selection and Constraints on Reheating, Phys.Rev. D85 (2012) 103533, [arXiv:1112.0326].

[32] J. Norena, C. Wagner, L. Verde, H. V. Peiris, and R. Easther, Bayesian Analysis of Inflation III: Slow Roll Reconstruction Using Model Selection, Phys.Rev. D86 (2012) 023505, [arXiv: 1202.0304].

[33] C. Ringeval, The exact numerical treatment of inflationary models, Lect. Notes Phys. 738 (2008) 243-273, [astro-ph/0703486].

[34] J. Martin and J. Yokoyama, Generation of Large-Scale Magnetic Fields in Single-Field Inflation, JCAP 0801 (2008) 025, [arXiv:0711.4307].

[35] V. Demozzi and C. Ringeval, Reheating constraints in inflationary magnetogenesis, JCAP 1205 (2012) 009, [arXiv: 1202.3022]. 
[36] S. Kuroyanagi, C. Ringeval, and T. Takahashi, Early Universe Tomography with CMB and Gravitational Waves, Phys.Rev. D87 (2013) 083502, [arXiv:1301.1778].

[37] C. Ringeval, T. Suyama, and J. Yokoyama, Magneto-reheating constraints from curvature perturbations, JCAP 1309 (2013) 020, [arXiv:1302.6013].

[38] Planck collaboration Collaboration, P. Ade et al., Planck 2013 results. XV. CMB power spectra and likelihood, arXiv:1303.5075.

[39] D. J. Schwarz, C. A. Terrero-Escalante, and A. A. Garcia, Higher order corrections to primordial spectra from cosmological inflation, Phys. Lett. B517 (2001) 243-249, [astro-ph/0106020].

[40] D. J. Schwarz and C. A. Terrero-Escalante, Primordial fluctuations and cosmological inflation after wmap 1.0, JCAP 0408 (2004) 003, [hep-ph/0403129].

[41] J. Martin, C. Ringeval, and V. Vennin, K-inflationary Power Spectra at Second Order, JCAP 1306 (2013) 021, [arXiv: 1303.2120].

[42] Planck Collaboration Collaboration, P. Ade et al., Planck 2013 results. I. Overview of products and scientific results, arXiv:1303.5062.

[43] A. Lewis and S. Bridle, Cosmological parameters from cmb and other data: a monte- carlo approach, Phys. Rev. D66 (2002) 103511, [astro-ph/0205436].

[44] A. Lewis, A. Challinor, and A. Lasenby, Efficient computation of cmb anisotropies in closed frw models, Astrophys. J. 538 (2000) 473-476, [astro-ph/9911177].

[45] D. Shepard, A two-dimensional interpolation function for irregularly-spaced data, in Proceedings of the 1968 23rd ACM national conference, ACM '68, (New York, NY, USA), pp. 517-524, ACM, 1968.

[46] W. I. Thacker, J. Zhang, L. T. Watson, J. B. Birch, M. A. Iyer, and M. W. Berry, Algorithm 905: Sheppack: Modified shepard algorithm for interpolation of scattered multivariate data, ACM Trans. Math. Softw. 37 (Sept., 2010) 34:1-34:20.

[47] A. Mazumdar and B. Zaldivar, Quantifying the reheating temperature of the universe, arXiv:1310.5143.

[48] A. A. Starobinsky, A New Type of Isotropic Cosmological Models Without Singularity, Phys.Lett. B91 (1980) 99-102.

[49] J. Ellis, D. V. Nanopoulos, and K. A. Olive, Starobinsky-like Inflationary Models as Avatars of No-Scale Supergravity, JCAP 1310 (2013) 009, [arXiv:1307.3537].

[50] J. Ellis, D. V. Nanopoulos, and K. A. Olive, No-Scale Supergravity Realization of the Starobinsky Model of Inflation, Phys.Rev.Lett. 111 (2013) 111301, [arXiv:1305.1247].

[51] J. P. Conlon and F. Quevedo, Kahler moduli inflation, JHEP 0601 (2006) 146, [hep-th/0509012].

[52] S. Krippendorf and F. Quevedo, Metastable SUSY Breaking, de Sitter Moduli Stabilisation and Kahler Moduli Inflation, JHEP 0911 (2009) 039, [arXiv:0901.0683].

[53] C. Burgess, M. Cicoli, and F. Quevedo, String Inflation After Planck 2013, JCAP 1311 (2013) 003, [arXiv: 1306.3512].

[54] A. Ijjas, P. J. Steinhardt, and A. Loeb, Inflationary paradigm in trouble after Planck2013, Phys.Lett. B723 (2013) 261-266, [arXiv:1304.2785].

[55] C. Germani and A. Kehagias, New Model of Inflation with Non-minimal Derivative Coupling of Standard Model Higgs Boson to Gravity, Phys.Rev.Lett. 105 (2010) 011302, [arXiv: 1003.2635]. 
[56] C. Germani and A. Kehagias, UV-Protected Inflation, Phys.Rev.Lett. 106 (2011) 161302, [arXiv: 1012.0853].

[57] C. Germani and Y. Watanabe, UV-protected (Natural) Inflation: Primordial Fluctuations and non-Gaussian Features, JCAP 1107 (2011) 031, [arXiv:1106.0502].

[58] T. Futamase and K.-i. Maeda, Chaotic Inflationary Scenario in Models Having Nonminimal Coupling With Curvature, Phys.Rev. D39 (1989) 399-404.

[59] M. B. Einhorn and D. T. Jones, Inflation with Non-minimal Gravitational Couplings in Supergravity, JHEP 1003 (2010) 026, [arXiv:0912.2718].

[60] W. Buchmller, V. Domcke, and K. Schmitz, Superconformal D-Term Inflation, JCAP 1304 (2013) 019, [arXiv: 1210.4105].

[61] R. Kallosh and A. Linde, Universality Class in Conformal Inflation, JCAP 1307 (2013) 002, [arXiv: 1306.5220].

[62] R. Kallosh and A. Linde, Superconformal generalization of the chaotic inflation model $\frac{\lambda}{4} \phi^{4}-\frac{\xi}{2} \phi^{2} R, J C A P 1306$ (2013) 027, [arXiv: 1306.3211].

[63] R. Kallosh and A. Linde, Superconformal generalizations of the Starobinsky model, JCAP 1306 (2013) 028, [arXiv: 1306.3214].

[64] S. Ferrara, R. Kallosh, A. Linde, and M. Porrati, Minimal Supergravity Models of Inflation, arXiv:1307.7696.

[65] R. Kallosh and A. Linde, Multi-field Conformal Cosmological Attractors, arXiv:1309. 2015.

[66] R. Kallosh, A. Linde, and D. Roest, A universal attractor for inflation at strong coupling, arXiv: 1310.3950.

[67] E. Silverstein and A. Westphal, Monodromy in the CMB: Gravity Waves and String Inflation, Phys. Rev. D78 (2008) 106003, [arXiv:0803.3085].

[68] A. de la Macorra and S. Lola, Inflation in S dual superstring models, Phys.Lett. B373 (1996) 299-305, [hep-ph/9511470].

[69] E. Dudas, N. Kitazawa, S. Patil, and A. Sagnotti, CMB Imprints of a Pre-Inflationary Climbing Phase, JCAP 1205 (2012) 012, [arXiv:1202.6630].

[70] E. Pajer, Inflation at the Tip, JCAP 0804 (2008) 031, [arXiv:0802.2916].

[71] N. Arkani-Hamed, H.-C. Cheng, P. Creminelli, and L. Randall, Pseudonatural inflation, JCAP 0307 (2003) 003, [hep-th/0302034].

[72] L. Boubekeur and D. Lyth, Hilltop inflation, JCAP 0507 (2005) 010, [hep-ph/0502047]. Latex, 20 pages, 5 figures. Minor changes, references added.

[73] P. Channuie, J. J. Jorgensen, and F. Sannino, Composite Inflation from Super Yang-Mills, Orientifold and One-Flavor QCD, Phys.Rev. D86 (2012) 125035, [arXiv:1209.6362].

[74] S. Kachru, R. Kallosh, A. D. Linde, and S. P. Trivedi, De Sitter vacua in string theory, Phys.Rev. D68 (2003) 046005, [hep-th/0301240].

[75] S. Kachru, R. Kallosh, A. D. Linde, J. M. Maldacena, L. P. McAllister, et al., Towards inflation in string theory, JCAP 0310 (2003) 013, [hep-th/0308055].

[76] L. Lorenz, J. Martin, and C. Ringeval, Brane inflation and the WMAP data: a Bayesian analysis, JCAP 0804 (2008) 001, [arXiv: 0709.3758].

[77] S. Clesse and J. Rocher, Avoiding the blue spectrum and the fine-tuning of initial conditions in hybrid inflation, Phys.Rev. D79 (2009) 103507, [arXiv:0809.4355].

[78] S. Clesse, C. Ringeval, and J. Rocher, Fractal initial conditions and natural parameter values in hybrid inflation, Phys. Rev. D80 (2009) 123534, [arXiv:0909.0402]. 
[79] S. Clesse, Hybrid inflation along waterfall trajectories, Phys.Rev. D83 (2011) 063518, [arXiv:1006.4522].

[80] S. Clesse and B. Garbrecht, Slow Roll during the Waterfall Regime: The Small Coupling Window for SUSY Hybrid Inflation, Phys.Rev. D86 (2012) 023525, [arXiv: 1204.3540].

[81] S. Clesse, B. Garbrecht, and Y. Zhu, Non-Gaussianities and Curvature Perturbations from Hybrid Inflation, arXiv:1304.7042.

[82] W. H. Kinney and A. Riotto, Dynamical supersymmetric inflation, Astropart.Phys. 10 (1999) 387-395, [hep-ph/9704388].

[83] W. H. Kinney and A. Riotto, A Signature of inflation from dynamical supersymmetry breaking, Phys.Lett. B435 (1998) 272-276, [hep-ph/9802443].

[84] F. Bezrukov, P. Channuie, J. J. Joergensen, and F. Sannino, Composite Inflation Setup and Glueball Inflation, Phys.Rev. D86 (2012) 063513, [arXiv:1112.4054].

[85] J. D. Barrow and P. Parsons, Inflationary models with logarithmic potentials, Phys.Rev. D52 (1995) 5576-5587, [astro-ph/9506049]. 